This document classified by:

Name R. E. Isaacson

Title Manager - Advanced

Technology Development

\title{
GEOCHEMICAL VARIATION OF COLUMBIA RIVER BASALTS \\ BENEATH THE HANFORD RESERVATION, WASHINGTON
}

\author{
J. B. Tatman \\ (NORCUS Participant) \\ Advanced Technology Development Section \\ Research and Development Department \\ Chemical Processing Division
}

September 30, 1972

This Report is Based on Work Sponsored by the Atlantic Richfield Hanford Company under United States

Atomic Energy Commission Contract AT(45-1)-2130 


\section{DISCLAIMER}

This report was prepared as an account of work sponsored by an agency of the United States Government. Neither the United States Government nor any agency Thereof, nor any of their employees, makes any warranty, express or implied, or assumes any legal liability or responsibility for the accuracy, completeness, or usefulness of any information, apparatus, product, or process disclosed, or represents that its use would not infringe privately owned rights. Reference herein to any specific commercial product, process, or service by trade name, trademark, manufacturer, or otherwise does not necessarily constitute or imply its endorsement, recommendation, or favoring by the United States Government or any agency thereof. The views and opinions of authors expressed herein do not necessarily state or reflect those of the United States Government or any agency thereof. 


\section{DISCLAIMER}

Portions of this document may be illegible in electronic image products. Images are produced from the best available original document. 
SUMMARY

Page

SUIMRY

INTRODUCTION

GEOLOGICAL SETTING

ANALYTICAL RESULTS

FACTORS INFLUENCING CHEMISTRY OF BASALTS 7

$\begin{array}{ll}\text { PRESENTATION OF CHEMICAL DATA } & 7\end{array}$

FREQUENCY DISTRIBUTION

CORRELATION COEFFICIENTS

TWO COMPONENT DIAGRAMS

Mgo Variation Diagram 8

ATkali-SiTica Diagram 9

THREE COMPONENT DIAGRAMS

$\mathrm{SiO}_{2}-\mathrm{FeO}-\mathrm{MaO}$

$\mathrm{FeO}-\mathrm{HgO}-\left(\mathrm{Na} 2 \mathrm{O}+\mathrm{K}_{2} \mathrm{O}\right) \quad 10$

$\mathrm{Na}_{2} \mathrm{O}-\mathrm{CaO}-\mathrm{K}_{2} \mathrm{O}$

FOUR COMPONENT DIAGRAMS

SOLIDIF ICATION INDEX

IRON ENRICHMENT DISCONTINUITY 11

STRATIGRAPHY OF BASALTS 12

$\begin{array}{ll}\text { CONCLUSIONS } & 13\end{array}$

$\begin{array}{ll}\text { ACKNOWLEDGEMENTS } & 13\end{array}$

$\begin{array}{ll}\text { REFERENCES } & 13\end{array}$

APPENDICES

APPENDIX A - MAJOR ELEMENT DATA

APPENDIX B - TRACE ELEMENT DATA

TABLES

FIGURES 


\section{LIST OF FIGURES}

Figure

1

2

3

4

5

6

7

8

9

10

11

12
Areal Distribution of Columbia River Basalts

Location of Core Holes

Histrograms of Averaged Geochemical Data

Mg0 Variation Diagram

Alkali-Silica Diagram

$\mathrm{SiO}_{2}$-FeO-HgO Diagram

$\mathrm{FeO}-\mathrm{MgO}-\left(\mathrm{Na}_{2} \mathrm{O}+\mathrm{K}_{2} \mathrm{O}\right)$ Diagram

$\mathrm{Na}_{2} \mathrm{O}-\mathrm{CaO}-\mathrm{K}_{2} \mathrm{O}$ Diagram

$\mathrm{SiO}_{2}-\mathrm{FeO}-\mathrm{MgO}-\mathrm{CaO}$ Diagram

$\mathrm{CaO}-\mathrm{FeO}-\mathrm{MgO} \mathrm{M} \mathrm{Al}_{2} \mathrm{O}_{3}$ Diagram

Iron Enrichment Diagram

Geochemical Variation of Columbia River Basalts Through Time 


\section{LIST OF TABLES}

Table

1 Major Element Averages and Ranges of Units $A-L$

2 Trace Element Averages of Units A-I

3 Correlation Coefficients for Major and Trace Elements

4 Solidification Indices of Units $A-L$

5 Stratigraphic Section of Columbia River Basalts 
SUMMARY

Major element and trace element analyses of Columbia River basalts have been compiled to indicate where geochemical changes occur in the stratigraphy of the basalts. The analyses were averaged and plotted on petrologic variation diagrams to elucidate geochemical trends and correlations. More work needs to be performed on the trace element data since this report is concerned mainly with the major elements.

\section{INTRODUCTION}

In'1969 the Atlantic Richfield Hanford Company initiated a study of the basalt stratigraphy of the Pasco Basin. Washington. The objective of the present study is to describe the major and trace element data acquired to date to foster a better understanding of the geochemical variations in the Columbia River basalts. The available geochemical data was compiled and diagrams illustrating chemical trends in the basalts are presented in the report.

\section{GEOLOGICAL SETTING}

The Columbia River Plateau is an intermontane basin occupied by miocene to pliocene tholeiitic basalts of southeastern Washington, northern Oregon, and portions of western Idaho. The plateau is bounded by the Okanogan Highlands to the north, the Blue Mountains to the south, the Rocky Mountains to the east, and the Cascade Range to the west. The Columbia River basalts cover an area of 200,000 square kilometers, have a volume of 195,000 cubic kilometers, and are one kilometer in average thickness (Kuno, 1969).

Plateau basalts are characterized by unusual thickness and great lateral extent of individual flows, and a paucity of pyroclastic material. Plateau basalts are formed from magmas representing advanced stages of low pressure fractional crystallization of partial melts from upper mantle peridotite and are erupted through fissures fomed by tensional stress in the crust.

The Pasco Basin is, and has been for some time, the central and lowest portion of the Columbia River Plateau. Early flows occur in an overlap relationship with the pre-existing topography due to the flooding action of the basalt and later thick fluid flows have been shown to have overcome regional slopes by onlap (Schminke, 1967a). Stratigraphic evidence indicates the latest basalt flows have an offlap relationship with earlier flows and tend to be more restricted to the center of the basin than earlier flows. This offlap relationship is due to subsidence of the plateau and to decreasing volume and probably decreasing frequency of erupted basalts. The subsidence is in large part the result of adjustment to the migration of magma from beneath the plateau onto the surface of the plateau. On the basis of tectonic history, the thickest section of Columbia River basalts in the plateau would be expected to be in the Pasco Bas in area and should provide excellent information on the changes in chemistry of the basalts through time. Figure 1 illustrates the geographical distribution of the Columbia River basalt and the boundaries of the Chief Joseph Dike Swarm to the southeast. 
In order to obtain data on the subsurface stratigraphy, five core holes located on or near the United States Atomic Energy Commission Hanford reservation were drilled. Figure 2 shows the location of the holes, which vary in depth from 180 to 1,525 meters. These cores provide an unprecedented opportunity to study a continuous record of basaltic products, with good lateral control, in a thick pile of plateau basalts.

ANALYTICAL RESULTS

Approximately 750 drill core samples will be studied by the Atlantic Richfield Hanford Company to examine variations in the basalt stratigraphy (Brown, in press). Samples have been analyzed to determine the concentrations of major and trace elements, radiometric ages, isotopic concentrations of radionuclides, and mineralogic variation. The oxides (Si02, Ti02, $\mathrm{Al}_{203}, \mathrm{FeO}, \mathrm{MnO}, \mathrm{MgO}, \mathrm{CaO}, \mathrm{Na}_{2} \mathrm{O}, \mathrm{K} 2 \mathrm{O}$ ) and minor elements ( $\mathrm{Ba}, \mathrm{Sr}, \mathrm{Rb}$, $\mathrm{Ni}, \mathrm{Zn}, \mathrm{Cu}, \mathrm{Sb}$, and $\mathrm{Pb}$ ) were analyzed by atomic absorption spectrophotometry while the rare earth elements ( $\mathrm{La}, \mathrm{Sm}, \mathrm{Eu}$, and $\mathrm{Tb}$ ) and other trace elements ( $\mathrm{Co}, \mathrm{Sc}, \mathrm{Cr}, \mathrm{Hf}$, $\mathrm{Th}$, and $\mathrm{Ta}$ ) were analyzed by instrumental neutron activation analysis.

Five hundred and twenty-two whole rock major element analyses were recalculated to 100 percent anhydrous and have been compiled (Appendix A). The normalization of the analyses tended to eradicate variations in chemistry due to analytical error, which resulted in high or low totals, and reduced all analyses to a standard total. The normalization calculation changed the concentration of the individual oxides in proportion to their original concentration and in proportion to the difference between 100 and the actual analytical total. The relationships between the relative values of the oxides of an analysis are preserved by the normalization although the absolute values of the oxides are uncertain.

After recalculation to 100 percent, the analyses were listed in tables (Appendix A) with each analysis placed in its correct relative depth location within the drill hole. Significant changes in the proportion of two or more oxides between adjacent analyses were marked, persistent variations noted, and groups of similar analyses delineated. The tables of each drill hole were then compared. Groups of analyses, henceforth called units, which displayed similar distinctive concentrations of oxides were matched. Units which were unique or which could be correlated between two or more drill holes were given an alphabetical designation. "A" was assigned to the uppermost unit and "B", "C", etc., were assigned to stratigraphically lower units. The ranges in concentration and averages of each oxide were calculated for the unique and correlated units; these are listed in Table I. The number in parentheses above each set of ranges is the number of samples in that unit and in most cases is proportional to the stratigraphic thickness of the unit.

Trace elements can also be used to divide the basalt into units. Since in this study the trace element analyses are not as numerous as those for major elements, they have been used as a secondary system to verify the stratigraphic breaks based on major element chemistry. An examination of trace element data shows that a change in trace element abundances 
usually occurs at the positions indicated by major element data. Certain units may be further subdivided on the basis of trace element data. The averages for each unit are listed in Table 2 and the data is tabulated in Appendix B, compiled to correspond in stratigraphic position to the major element data of Appendix $A$.

\section{FACTORS INFLUENCING CHEMISTRY OF BASALTS}

In studying the chemistry of basalts it is found that other than stating that a basalt has a specific composition it is difficult to draw conclusions as to why the basalt does in fact have that specific composition. If a series of basalts does not show definite compositional trends which can be ascribed to, the crystallization of a specific mineral of which the basalt is more fundamental has to be sought.

The composition of an erupted basalt is influenced by the composition of the original liquid, the nature and proportion of solid phases crystallizing from the liquid, and the distribution of elements between solid phases and the liquid. The composition of the original liquid is dependent upon the composition of the source rock, the type, proportion, and composition of minerals in the source rock, the degree of partial melting, and the temperature and pressure at the site of magma generation. The nature and proportion of solid phases crystallizing depends upon the composition of the liquid and the temperature and pressure at the site of crystaliization. The density of the liquid and of the crystalline phases influences the gravitational settling of crystalline phases, and the viscosity of the magma influences the rate of upward migration of the magma. The volatile content of the magma influences the oxygen fugacity and the pressure and temperature of crystallization, and the composition of the country rock through which the magma passes may have a profound effect upon its chemistry. All of these factors play some part in influencing the composition of the erupted basalt.

PRESENTATION OF CHEMICAL DATA

\section{Frequency Distribution}

To show the frequency distribution of the averaged data from Tables I and II, 19 histograms were constructed for the oxides and trace elements of Columbia River basalts. A total of 36 whole rock major element analyses from Table I and 18 whole rock trace element analyses from Table II were utilized. Due to the small number of samples used, the type of distribution exhibited in many cases is often not distinct. However, from the diagrams constructed, $\mathrm{SiO}_{2}, \mathrm{TiO}_{2}, \mathrm{FeO}, \mathrm{Cr}$ and $\mathrm{Ba}$ show notably bimodal mixed distributions while $\mathrm{Al}_{20} \mathrm{O}_{3}, \mathrm{CO}$, Hf and Th show normal distributions and $\mathrm{K}_{2} \mathrm{O}$ and $\mathrm{Ta}$ show positively skewed normal distributions. The other oxides and trace elements have no discernible distinct distribution patterns. The process or processes which determine the creation of the observed frequency distributions have not been determined. 
Correlation Coefficients

A correlation coefficient is a number between 1.0 and -1.0 which measures the dependency between two variables. A value of 1.0 indicates identity or absolute positive association while a value of -1.0 indicates absolute negative association. A value of 0.0 indicates neither an affinity nor a non-affinity. Coefficients of absolute magnitude smaller than unity indicate a degree of relative association depending upon their value; a value of -.20 indicates a weak negative correlation, while a value of .75 indicates a strong positive correlation.

From the correlation coefficients of the oxides it is observed that MgO has a positive correlation with $\mathrm{Al}_{2} \mathrm{O}_{3}$ and $\mathrm{CaO}$ and a negative correlation with $\mathrm{SiO}_{2}, \mathrm{TiO}_{2}, \mathrm{FeO}, \mathrm{Na}_{2} \mathrm{O}$ and $\mathrm{K}_{2} \mathrm{O}$; the same relationships which are observed for the oxides from the MgO variation diagram. The correlation coefficients express a more quantitative aspect of the associations, while the variation diagram expresses a more graphical aspect. Compared to variation diagrams, correlation coefficients are more versatile since they express relations between all the components to one another rather than to a standard component such as MgO. However, it is more difficult to grasp the relations since they are in numerical form.

It will be left to the reader to ascertain the various relations between oxides and elements other than noting an association between $\mathrm{Sc}, \mathrm{Cr}$, and $\mathrm{Co}$ and a strong association between $\mathrm{Ba}, \mathrm{La}, \mathrm{Sm}, \mathrm{Eu}, \mathrm{Tb}, \mathrm{HF}, \mathrm{Ta}$, and Th; there being a negative relation between the two groups.

\section{TWO COMPONENT DIAGRAMS}

\section{Mgo Variation Diagram}

Variation diagrams are used to illustrate the change in elemental composition of a suite of rocks to infer the composition of the melt from which they were derived. In this study variation diagrams with $\mathrm{MgO}$ as the abscissa and the other oxides (Si02, Ti02, $\mathrm{Al}_{2} \mathrm{O}_{3}, \mathrm{FeO}_{2}$ $\mathrm{CaO}, \mathrm{Na}_{2} \mathrm{O}$ and $\mathrm{K}_{2} \mathrm{O}$ ) as the ordinate have been constructed using the averages from the designated units of the five core holes listed in Table I. The lines connecting points on the diagrams are meant to identify average compositions belonging to the same unit and do not represent the field of compositions in which all the analyses from a specific unit lie. The Mg0 variation diagram was chosen as the method to illustrate single element variation of Columbia River basalts since the change in composition of the melt due to the influence of the crystallization of individual minerals (olivine, pyroxene, plagioclase, and iron-titanium oxide) is more readily discernible in this diagram than in other types of variation diagrams.

During the evolution of tholeioitic basalts large quantities of olivine may crystallize and settle out by gravitative differentiation (MacDonald, 1968 ). The crystallization of olivine alters the composition of the liquid phase 
at higher temperature while pyroxene, plagioclase, and iron-titanium oxide alter it at lower temperature. The use of Mgo variation diagrams permits a linear extrapolation of a liquid line of descent trend; controlled by olivine at the high MgO end and by pyroxene, plagiociase and iron-titanium oxide at the low MgO end.

When an individual phase crystallizes it preferentially concentrates certain elements into its structure, different phases concentrating different proportions of elements. If the concentrations of the elements incorporated into the crystal are less than the concentration in the liquid, they will increase in concentration in the liquid. If the concentrations of the elements in the crystal are more than that in the liquid, they will decrease in concentration in the liquid. For example, when a magnesium-rich olivine crystallizes from a liquid of basaltic composition, the liquid becomes depleted in "gig and enriched in $\mathrm{SiO}_{2}$ and $\mathrm{FeO}$. From the MgO variation diagram it is observed that as $\mathrm{MgO}$ decreases $\mathrm{SiO}_{2}, \mathrm{TiO}_{2}, \mathrm{FeO}_{2}, \mathrm{Na}_{2} \mathrm{O}$ and $\mathrm{K}_{2} \mathrm{O}$ increase while $\mathrm{Al}_{2} \mathrm{O}_{3}$ and $\mathrm{CaO}$ decrease.

\section{Alkali-Silica Diagram}

The $\mathrm{SiO}_{2}-\left(\mathrm{Na}_{2} \mathrm{O}\right.$ and $\left.\mathrm{K}_{2} \mathrm{O}\right)$ diagram delineates the fields of alkalic basalt and tholeiitic basalt as observed by MacDonald and Katsura (120.4) from Hawaiian basalts (Figure 5). The insert is a diagram by Kuno (1965) illustrating the compositional trends of basalts from various localities. Al1 the average compositions of units of the Columbia River basalts plot within the tholeite field.

\section{THREE COMPONENT DIAGRAMS}

\section{Silica-Iron-Magnesium Diagram}

The Si02-FeO-MgO diagram describes the behavior of mafic and ultramafic rocks better than most ternary diagrams since the degree of explanation, indicated by the sum of the values of the three oxides before normalization to 100 , is generally greater than 70 percent (Figure 6). The Si02-Fe0-MgO diagram illustrates the compositional fields of rocks characteristic of the upper mantle (Therzolite, websterite, and eclogite) and of rocks derived from them (basalt, basanite and nephelinite). The skaergaard trend is the path of liquids from a differentiated gabbroic intrusion. The Columbia River basalts lie in a distinct area between the fields of the Cascade Range basalts and the Snake River basalts, indicating they have relatively less silica than the Cascade basalts and relatively less iron than the Snake River basalts.

The minerals olivine, orthopyroxene, and clinopyroxene are located near the Si02-MgO edge at approximately 40, 60 and 73 percent, respectively. As the liquid changes in composition the crystals precipitated plot in a line extending from magnesian compositions to relatively more ironrich and silica-poor compositions. Plagioclase lies at the Si02 apex and magnetite and ilmenite at the FeO apex. A partial melt derived from 
Therzolite has a composition near the websterite and eclogite fields from which it may evolve towards basalt by crystallizing olivine and pyroxene. Plagioclase precipitation in Columbia River basalts does not seem to have influenced the composition of the liquid until late stage, near-surface crystallization.

\section{Iron-Magnesium-A7ka7r Diagram}

The $\mathrm{FeO}-\mathrm{HgO}-\left(\mathrm{Na}_{2} \mathrm{O}\right.$ and $\left.\mathrm{K}_{2} \mathrm{O}\right)$ diagram shows Columbia River basalts lie within the area characteristic of tholelites (Figure 7). The Hawaitan tholeitte and skaergaard trends are after MacDonald and Katsura (1964). Plagioclase occupies the $\mathrm{Na}_{2} \mathrm{O}-\mathrm{K}_{2} \mathrm{O}$ apex, while iron-titanium oxides occupy the $\mathrm{Fe} 0$ apex. OTivine and orthopyroxene are clustered very close together near the 75 to 85 percent Mgo position along the FeO-MgO edge. Clinopyroxene lies near 01 ivine and orthopyroxene but is displaced about 5 percent toward the $\mathrm{Na}_{2} \mathrm{O}-\mathrm{K}_{2} \mathrm{O}$ apex. On this diagram it is difficult to evaluate the influence on the composition of the liquid by the separate crystallization of the individual major mafic minerals since they plot so close together. However, from their position on the diagram it can be observed that the crystallization of olivine and pyroxene is responsible for much of the compositional trend of the basalts.

Sodium-Calcium-Potassium Diagram

The $\mathrm{Na}_{2} \mathrm{O}-\mathrm{C}_{2} \mathrm{O}-\mathrm{K}_{2} \mathrm{O}$ diagram is frequently used to illustrate the fractionation trend for more differentiated rocks (trachytes, phonolytes and rhyolites) (Figure 8 ). The trend described by Columbia River basalts generaliy coincides with that for tholeititic basalts. Plotted along the Na20-CaO edge is the mole percent anorthite content of plagioclase. Clinopyroxene plots near the CaO apex and the compositional trend of the analyses indicates $\mathrm{CaO}$ has produced the majority of the change. Plagioclase and/or clinopyroxene crystallization could, therefore, have produced much of the trend.

\section{FOUR COMPONENT DIAGRAMS}

The quaternary diagrams $\mathrm{S}_{7} \mathrm{O}_{2}-\mathrm{Fe} 0-\mathrm{Plg} \mathrm{O}-\mathrm{CaO}$ and $\mathrm{CaO}-\mathrm{Fe} 0-\mathrm{MgO}-\mathrm{Al}_{2} \mathrm{O}_{3}$ describe the chemical relations of Columbia River basalts within four component systems. The two diagrams 11 llustrate an altered tetrahedron with three of the faces modified to coincide with the plane of the base of the tetrahedron. The position of the analyses of the basalts projected onto the four faces of the tetrahedron indicate their relative compositional trends.

The $\mathrm{SiO}_{2}-\mathrm{Fe} 0-\mathrm{MgO}-\mathrm{CaO}$ diagram shows a compositional trend in which the basalts generaliy decrease in $\mathrm{MgO}$ and $\mathrm{CaO}$ and increase in $\mathrm{FeO}$ and $\mathrm{SiO} 2$ with differentiation. The $\mathrm{CaO}-\mathrm{Fe} 0-\mathrm{Mg} 0-\mathrm{Al}_{2} \mathrm{O}_{3}$ diagram shows a general decrease in $\mathrm{MgO}$ and increase in $\mathrm{FeO}$. CaO decreases siightiy in relative composition, while $\mathrm{Al}_{2} \mathrm{O}_{3}$ increases in the absence of $\mathrm{Fe} 0$ and decreases when it is present. 
The degree to which the basalt compositions are explained is, as in ternary diagrams, the sum of the values of the oxides comprising the diagram. The manner in which compositions were projected onto the faces was by calculating three oxides at a time, in essence, constructing four ternary diagrams. Therefore the degree of explanation in this case does not equal the sum of the oxides since the four oxides are never simultaneously used to calculate the diagram. A better mode of representing compositions within a quaternary diagram would be by producing a three-dimensional, stereographic illustration of a tetrahedron in which compositions would be represented as one point instead of four. However, until such a diagram is made available the present system will have to suffice.

\section{SOLIDIFICATION INDEX}

The solidification index $\left[\mathrm{MgO} \times 100 /\left(\mathrm{MgO}+\mathrm{Fe} 0+\mathrm{Fe}_{2} \mathrm{O}_{3}+\mathrm{Na}_{2} \mathrm{O}+\mathrm{K}_{2} \mathrm{O}\right)\right]$ is the percentage of $M g 0$ of a composition plotted in the iron-magnesiumalkali diagram and can be employed as an approximation of the degree of solidification which primary magma underwent to produce the observed composition. Table IV is a listing of the solidification indices of the average compositions of units of Columbia River basalt.

The solidification index of a magma decreases as fractionation proceeds, and it is assumed the decrease is proportional with the amount of residual Tiquid. Primary magmas produced by partial melting in the upper mantle have solidification indices of 40 or higher. If 40 is taken as a general index for primary magmas, basalts with an index of 20 should correspond to magmas produced by 50 percent solidification (KunD, 1969). Dr. A. C. Waters has also pointed out the usefulness of this method of correlating basalts in an unpublished report dated June 23, 1972 to the Atlantic Richfield Hanford Company.

\section{IRON ENRICHMENT DISCONTINUITY}

The iron enrichment $[\mathrm{FeO} /(\mathrm{Fe} 0+\mathrm{MgO})]$ diagram of Columbia River basalts illustrates a chemical discontinuity between .62 and .68 of the ironmagnesium ratio (Figure 11). Experimental evidence (Tilley and Thompson, 1970) indicates that at one atmosphere pressure the liquidus tempera'we of basalts decreases as iron enrichment increases. In order to explain such a gap exhibited by many volcanic suites, Thompson (1972) used the conclusions of Wright and Fiske (1971) from their study of lavas from Kilauea, Hawaif. Thompson believes the chemical discontinuity is generated as follows: basaltic magma is formed by partial melting of the upper mantle. Some of this magma is erupted unmodified except for the chemical variation resulting from high pressure partial melting trends and low pressure olivine subtraction or addition. Some batches of magma stagnate on the way to the surface and form irregular and sill like bodies which crystallize until they are approximately 50 to 60 percent solidified. At this point the crystal meshwork of the magma batch is sufficiently rigid to fracture without disaggregation when stressed, allowing the differentiated, low liquidus temperature liquid to migrate to lower pressure and be erupted with little further modification. 
This model explains the chemical discontinuity as the result of a repetitive, repraducible, discontinuous sampling process superimposed on other differentiation processes. This process allows only relatively unevolved basalts and basalts from highly evolved interstitial liquids to be erupted (Thompson, 1972). Basalts of Units B and D would, therefore, belong to the first group while all the other basalts of the other units would belong to the second group, except Unit $K$ of $D H-3$ which is transitional.

\section{STRATIGRAPHY OF BASALTS}

The stratigraphic section of the Columbia River basalt is presently divided into two major sections, the younger Yakima basalts and the older Picture Gorge basalts. Table $V$ is the nomenclature of Columbia River basalt stratigraphy as defined by Schminke (1976b) and Wright, Grolier, and Swanson (in press), with minor modifications.

The chemistry of subsurface basalt units from the cored holes has been compared with analyses from type localities. The uppermost portion of Unit A from core hole DH-3 corresponds to Ice Harbor, while Unit A itself is equivalent to Elephant Mountain. Unit B corresponds to Pomona, Unit $C$ to the lower flow exposed on Gable Mountain, Unit D to Huntzinger, and Unit $E$ is equivalent to Umatilla. Unit $F$ is correlated with Priest Rapids and Roza, Unit $G$ with Frenchman Springs, and Unit $H$ corresponds to Museum and Rocky Coulee.

Units $G, H$ and I are quite thick, indicating minor change in chemistry with massive eruption of basalt. This is in contrast to the variability of basalt chemistry above and below these thick units. Basalts of Units $\mathrm{J}$ and $\mathrm{K}$ from $\mathrm{DH}-3$ are not correlated with those from core holes $\mathrm{DH}-4$ and $\mathrm{DH}-5$. They are grouped together for convenience and this should be taken into consideration. Instead of the 12 units listed in the table, there should actually be three more due to the uniqueness in DH-3 of Units $J, K$ and upper $A$. This study has, therefore, defined 15 different geochemical-stratigraphic units.

The basalt units defined in this study are frequently comprised of more than one flow unit as indicated by lithologic contact indicating the top or bottom of a flow. This indicates that a geochemical basalt unit does correspond to a distinct sequence of basalt flow units.

Figure 12 is an illustration of the geochemical variation of Columbia River basalt through time; Unit $A$ is the youngest and Unit $L$ is the oldest basalt unit studied. With decreasing age the composition of the basalts generally decreases in $\mathrm{SiO}_{2}, \mathrm{Al}_{2} \mathrm{O}_{3}, \mathrm{Na}_{2} \mathrm{O}, \mathrm{K}_{2} \mathrm{O}$ and increases in $\mathrm{TiO}_{2}, \mathrm{FeO}, \mathrm{CaO}$ and $\mathrm{MgO}$. 


\section{CONCLUSIONS}

The problem of correlating basalts over extended areas is greatly facilitated by chemical data and promises to be the most definitive method of correlation.

The data of this study indicate the Pomona magma type (Units B and D) is more primitive in chemical composition than other Yakima basalt units, while the Umatilla magma type (Unit E) is the most differentiated basalt studied.

The key to understanding the chemistry of basalts seems to be in the process of the generation of magma from source material rather than the process of fractional crystallization. The crystallization process does play an important role in understanding the chemistry of the basalts but it does not fully explain the compositional variability of basalts. Therefore a more fundamental cause must be sought and it seems to be in the process of melting.

\section{ACKNOWLEDGEMENTS}

The work was done during the summer of 1972 while the author was an appointee to the Northwest College and University Association for Science laboratory graduate program. The chemical analyses of the basalts and several unpublished reports on basalt flow correlation methods were generously made available by Messrs. R. E. Isaacson and D. J. Brown of Atlantic Richfield Hanford Company. The author is also indebted to Messrs. Brown and R. K. Ledgerwood for valuable information and helpful advice. The four component diagrams were produced by a computer plotting program written by Dr. Steven Robinson of the University of Washington. Mr. R. E. Isaacson and Dr. M. D. Veatch kindly reviewed the manuscript.

\section{REFERENCES}

Brown. D. J., "Identifiable Basalt Flows in the Pasco Basin, Washington," (in press).

Frey, F. A., M. A. Haskin, J. A. Poetz and L. A. Haskin, "Rare Earth. Abundances in Some Basic Rocks," Jour. Geoph. Res., 73, pp. 6085-7003.

Goles, G. G. and M. Osawa "Petrogenesis of Columbia River Basalts," Trans. Am. Geoph. Un., 52, p. 375, (1971).

Hi11, D. P., "Crustal and Upper Mantle Structure of the Columbia River Plateau from Long-Range Seismic-Refraction Measurements," Bu11. Geo1. Soc. Am., 83, pp. 1639-1648, (1972).

Hubbard, N. J., "A Chemical Comparison of Oceanic Ridge, Hawaiian. . Tholeijtic and Hawaijan Alkalic Basalts," Earth Planet Sci. Lett., 5 , pp. 346-352, (1969). 
REFERENCES (Continued)

Kuno, H., "Fractionation Trends of Basalt Magmas in Lava Flows," Jour. Petrol., 6, pp.302-321, (1965).

-...-. "Plateau Basalts in the Earth's Crust and Upper Mantle," Am. Geoph. Un. Mono., 13, pp. 495-501, (1969).

MacDona1d, G. A., "Composition and Origin of Hawaijan Lavas,"

In Studies in Volcanology, Geol. Soc. Am. Mem. 116, pp.477-522, (1968).

MacDonald, G. A. and T. Katsura, "Chemical Composition of Hawairan Lavas, "Jour. Petro1., 5, pp.82-133, (1964).

Osawa, M. and G. G. Goles, "Trace Element Abundances in Columbia River Basalts," Proc. Second Columbia River Basalt Symposium, Eastern Washington State College Press, pp.73-96, (1970).

Powers, H. A., "A Distinctive Chemical Characteristic of Snake River Basalts of Idaho," U. S. Geol. Surv. Prof.Paper 400B, 298, (1960).

Schmincke, $H_{0}$ U. "Flow Directions in Columbia River Basalt Flows and Paleocurrents of Interbedded Sedimentary Rocks of South-Central Washington," Geol. Rundschau, 56, pp.992-1020, (1967a).

-.-.-. "Stratigraphy and Petrology of Four Upper Yakima Basalt Flows in South-Central Washington," Bu11.Geol. Soc. Am., 78, pp. 106-110, (1967b).

Taubeneck, W. H., "Dikes of Columbia River Basalt in Northeastern Oregon, Western Idaho, and Southeastern Washington, "Proc. Second Columbia River Basalt Symposium, Eastern Washington State College Press, pp. 73-96, (1970)。

Thompson, R. N., "Evidence for a Chemical Discontinuity Near the BasaltAndesite Transition in Many Anorgenic Volcanic Suites," Nature, 236, pp. 106-110, (1972)。

Tilley, C. E。 and R. N. Thompson, "Melting and Crystallization Relations of the Snake River Basalts of Southern Idaho," Earth Planet. Sci. Lett, 8, pp. 79-92, (1970)。

Waters, A. C., "Stratigraphic and Lithologic Variations in the Columbia River Basalt," Am. Jour.Sci., 259, pp. 593-611, (1961).

-...--, unpublished to Atlantic Richfield Hanford Company, (1972).

Wright, T. L., M. J. Groljer and D. A. Swanson, "Chemical Variation Related to the Stratigraphy of the Columbia River Basalt, "Bul1. Geol. Soc. Am. 84, pp. 371-386, (1972). 
APPENDTX A

Data in parts per million 
DH-I

S102 T102 AL2O3 CR2O3 FE2O3 FEO MNO MGO CAO NA2O K2O

$\begin{array}{lllllllllll}C A 1 & 53.3 & 3.2 & 12.6 & 14.4 & 2 & 4.4 & 8.1 & 2.4 & 1.4\end{array}$

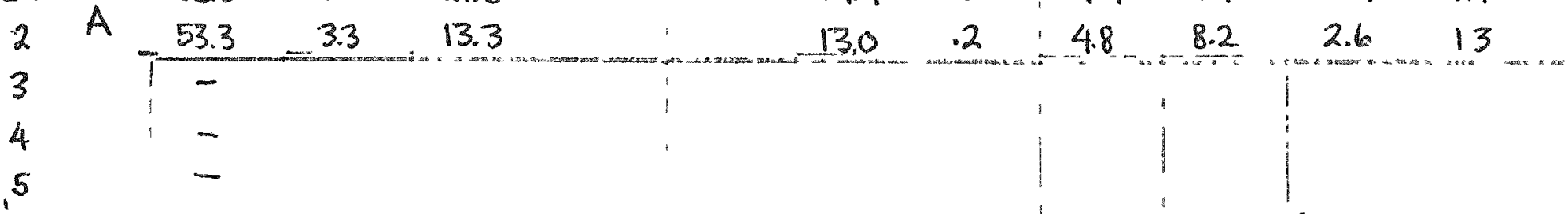

$\begin{array}{llll}6 & 54.6 & 2.0 & 14.9\end{array}$

$\begin{array}{llll}1030 & 55.6 & 1.6 & 14.8\end{array}$

$\begin{array}{llll}7 & 54.6 & 2.0 & 14.3\end{array}$

$\begin{array}{lllll}8 & B & 54.1 & 1.8 & 14.8\end{array}$

$\begin{array}{llll}1031 & 54.0 & 1.7 & 14.7\end{array}$

$\begin{array}{llll}9 & 52.4 & 1.8 & 14.5\end{array}$

$1033 \quad 53.4 \quad 1.6 \quad 13.8$

$\begin{array}{llll}10 & 51.7 & 20 & 14.1\end{array}$

$\begin{array}{llll}1034 & 52.1 & 1.6 & 14.8\end{array}$

$\begin{array}{llll}11 & 51.7 & 1.9 & 14.6\end{array}$

$\begin{array}{llll}12 . & 51.8 & 1.9 & 14.3\end{array}$

$\begin{array}{llll}1035 & 51.7 & 1.5 & 14.5\end{array}$

$\begin{array}{llll}13 & -51.9 & 18 & 14.7\end{array}$

$\begin{array}{llll}11.1 & .2 & 5.5 & 9.4\end{array}$

$1.8 \quad .5$ $\begin{array}{lllll}10.2 & 5.6 & 9.7 & 2.1 & .4\end{array}$

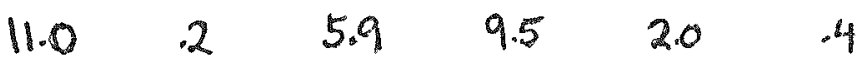
$\begin{array}{llllll}10.6 & .2 & 6.0 & 9.5 & 2.4 & .6\end{array}$ $\begin{array}{lllll}10.6 & 6.0 & 10.1 & 2.4 & .4\end{array}$ $\begin{array}{llllll}10.6 & .2 & 7.2 & 10.6 & 2.3 & .5\end{array}$ $\begin{array}{lllll}11.0 & 7.5 & 10.3 & 2.3 & .3\end{array}$ $\begin{array}{llllll}11.9 & .2 & 7.0 & 10.3 & 2.3 & .6\end{array}$ $\begin{array}{lllll}10.9 & 80 & 9.6 & 2.5 & 5\end{array}$ $\begin{array}{llllll}10.6 & .3 & 6.8 & 11.0 & 2.5 & .6\end{array}$ $\begin{array}{llllll}11.7 & .2 & 7.3 & 9.9 & 2.5 & .5\end{array}$ $\begin{array}{lllll}11.0 & 8.7 & 9.8 & 2.3 & .4\end{array}$

14

11.5 $7.0 \quad 10.1$ 2.3 .4

$15 \quad-$

16

$\begin{array}{llll}17 & 55.9 & 2.9 & 13.3\end{array}$

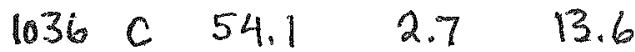

$\begin{array}{llll}1037 & 53.9 & 2.5 & 13.7\end{array}$

$\begin{array}{llll}18 & 54.0 & 2.8 & 13.2\end{array}$

$\begin{array}{llll}1038 & 53.2 & 2.7 & 13.2\end{array}$

$\begin{array}{llll}19 & 53.8 & 28 & 13.2\end{array}$

$\begin{array}{llll}1039 & 54.2 & 2.7 & 13.3\end{array}$

$\begin{array}{llllll}2.1 & .2 & 3.7 & 7.2 & 2.9 & 1.7\end{array}$

$20 \quad \frac{53.2}{21} \quad 2.8 \quad 13.2$

$\begin{array}{llll}1040 & 52.1 & 1.5 & 15.1\end{array}$

$\begin{array}{lllll}22 & D & 51.8 & 1.6 & 14.8\end{array}$

$\begin{array}{llll}1041 & 51.1 & 15 & 15.2\end{array}$

$\begin{array}{llll}1042 & 52.5 & 1.4 & 14.8\end{array}$

$\begin{array}{llll}23 & 52.2 & 1.8 & 14.7\end{array}$

$\begin{array}{llll}2.4 & 54.4 & 3.5 & 16.0\end{array}$

$\begin{array}{llll}25 & 56.8 & 28 & 14.2\end{array}$

$\begin{array}{llll}1043 & 56.5 & 2.3 & 137\end{array}$

$\begin{array}{lllll}14.1 & 4.2 & 7.5 & 2.5 & 1.3\end{array}$

$\begin{array}{lllll}13.5 & 4.2 & 7.4 & 2.8 & 2.1\end{array}$

$\begin{array}{llllll}13.4 & .2 & 4.1 & 75 & 29 & 2.0\end{array}$

$\begin{array}{lllll}139 & 4.1 & 88 & 27 & 15\end{array}$

$\begin{array}{llllll}14.2 & .2 & 3.9 & 7.3 & 2.9 & 1.6\end{array}$

$\begin{array}{lllll}13.9 & 4.3 & 82 & 2.8 & \text { i6 }\end{array}$

$\begin{array}{llllll}14.7 & .2 & 3.9 & 7.3 & 29 & 1.7\end{array}$

$\begin{array}{llllll}11.8 & 1 & 6.2 & 9.7 & 23 & .7\end{array}$

$\begin{array}{lllll}10.8 & 7.7 & 9.8 & 2.4 & .5\end{array}$

$\begin{array}{llllll}11.8 & .2 & 7.0 & 9.2 & 2.4 & 1.1\end{array}$

$\begin{array}{lllll}11.0 & 8.7 & 9.4 & 2.4 & .7\end{array}$

$\begin{array}{lllll}10.6 & 8.3 & 91 & 2.3 & .9\end{array}$

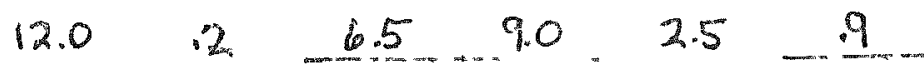

$\begin{array}{llllll}144 & 1 & 20 & 5.3 & 28 & 1.6\end{array}$

$\begin{array}{llllll}11.7 & 2 . & 2.5 & 63 & 3.0 & 25\end{array}$

$\begin{array}{lllll}12.4 & 28 & 6.1 & 33 & 2.8\end{array}$ 
DH-I

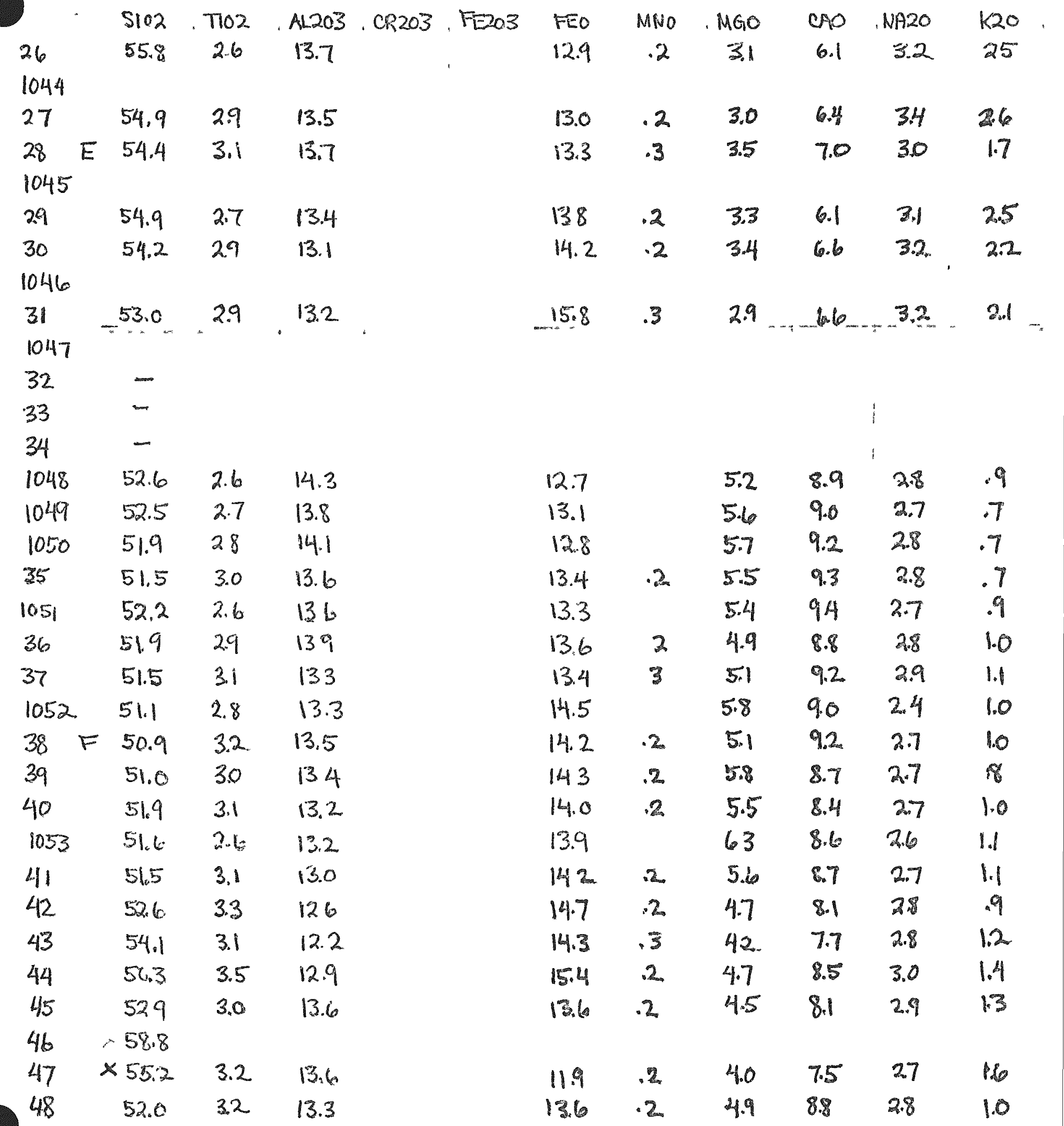




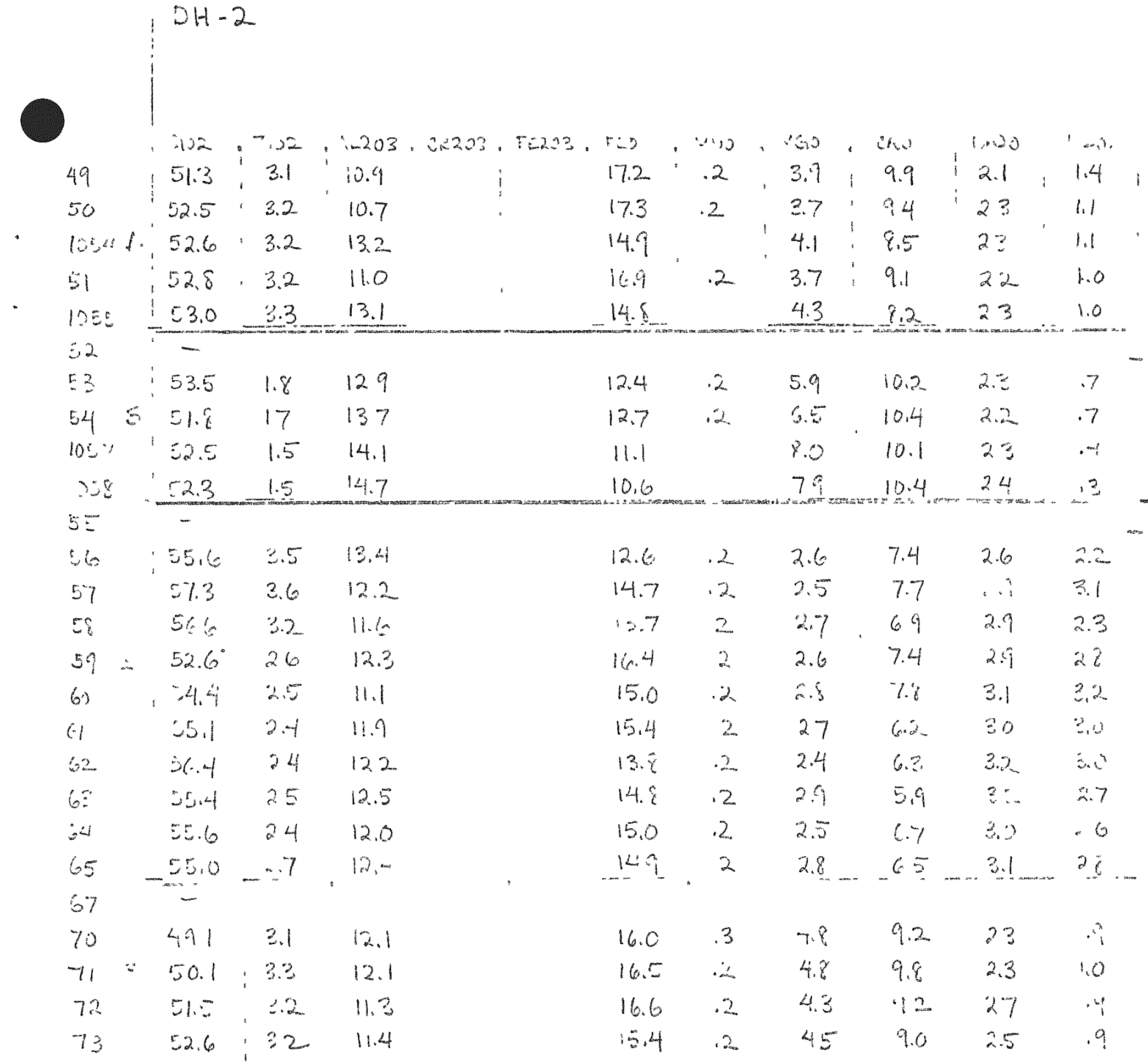


DH.3

\begin{tabular}{|c|c|c|c|c|c|c|c|c|}
\hline & 102 & 7102 & ALCE, , =03, FEan? & 9100 & Hao & $M$ & $15 \% 20$ & $=20$ \\
\hline 2001 & 50.5 & 31 & .36 & 136 & 5.9 & 10.6 & 20 & .7 \\
\hline 2 & 50.8 & 33 & 132 & 131 & 65 & 10.1 & 2.2 & 7 \\
\hline 3 & 51.8 & 3.2 & 12.9 & 13.1 & 6.0 & 10.1 & 24 & .6 \\
\hline 152 & 52.0 & 22 & 12.9 & 15.2 & 5.0 & 8.2 & 27 & .7 \\
\hline 61 & 529 & 21 & 12.2 & 141 & 5.2 & 8.7 & 24 & 1.4 \\
\hline 153 & 52.3 & 32 & 12.8 & 15.5 & 4.9 & 7.9 & 2.6 & .8 \\
\hline 8 & 53.2 & 33 & 12.6 & 14.1 & 44 & 8.8 & 24 & 10 \\
\hline 9 & 52.7 & 34 & 11.8 & 15.3 & 44 & $7 \delta$ & 22 & 22 \\
\hline 154 & 521 & 3.1 & 121 & 15.3 & 48 & 79 & 28. & 8 \\
\hline 11 & 542 & 17 & 13.3 & 111 & 71 & 10.1 & 21 & 4 \\
\hline 12 & 55.3 & 1.6 & 13.4 & 110 & 7.1 & 8.8 & 22 & .6 \\
\hline 13 & 54.8 & 1.6 & 136 & 12.9 & 72 & 91 & 23 & 5 \\
\hline 14 & $=5.5$ & 1.7 & 124 & 106 & 73 & 87 & 23 & .6 \\
\hline 16 & 57.1 & 2.7 & 133 & 139 & 36 & 6.0 & 20 & 13 \\
\hline 17 & 55.1 & 27 & 12.3 & 14.4 & 4.1 & 72 & 25 & 1.7 \\
\hline 18 & 55.4 & 2.4 & 12.9 & 136 & 3.9 & 76 & $2 \%$ & 15 \\
\hline 19 & 55.0 & 26 & 129 & 144 & 37 & 72 & 26 & 17 \\
\hline $2<$ & $=56.4$ & 28 & $12 c$ & 13.2 & 3.7 & 61 & 29 & $=2$ \\
\hline$\therefore 4$ & 57.4 & 24 & 138 & 18 & 2.9 & 5.7 & $=3$ & 25 \\
\hline 25 & 57.4 & 23 & 134 & 12.1 & 29 & 60 & 32 & 27 \\
\hline 25 & 57.9 & 2.3 & 13.7 & 12.1 & 2.6 & 5.6 & 3.1 & 26 \\
\hline 27 & 57.3 & 2.3 & 131 & 12.7 & 29 & 5.7 & 34 & 26 \\
\hline $2 \varepsilon$ & 57.4 & 2.2 & 128 & $12 . \%$ & 3.0 & 5.8 & 34 & 2.6 \\
\hline 29 & 563 & 2.3 & 12.6 & 125 & 44 & 6.0 & 33 & 25 \\
\hline 31 & 541 & 2.6 & 128 & 147 & 4,0 & $? 1$ & 24 & 2 \\
\hline 32 & 53.1 & 26 & 123 & 14.5 & 5.2 & 88 & 2.5 & .9 \\
\hline 33 & 53.2 & 26 & 13.1 & 136 & 5.0 & 8.7 & 24 & 9 \\
\hline 34 & -532 & 2.7 & 131 & 13.6 & 5.2 & 8.4 & 27 & $1 . i$ \\
\hline 155 & $\because 50.5$ & 32 & 15.3 & 14.1 & 4.7 & 85 & 31 & .6 \\
\hline 36 & 53.6 & 2.6 & 132. & 14.1 & 44 & 26 & 2.5 & .9 \\
\hline 31 & 53.1 & 2.6 & 13.2 & 14.2 & 48 & 8.5 & 26 & 6 \\
\hline $3 \tilde{\varepsilon}$ & 537 & 2.5 & 13,3 & 13.9 & 4.4 & 84 & 27 & 11 \\
\hline 34 & 53.4 & 2.7 & 12.8 & 14.4 & 4.7 & 8.1 & $2 \%$ & 1.2 \\
\hline 40 & 542 & 2.5 & 12.9 & 13.8 & 4.4 & 8.4 & 26 & 12 \\
\hline $4 i$ & 05.2 & 2.7 & 12.6 & 14.1 & 4.1 & 78 & 25 & 11 \\
\hline 9.9 & 54.5 & 24 & $12 k$ & 146 & 4.4 & 7.6 & $2 \%$ & 12 \\
\hline 31 & 54.1 & 30 & 12.4 & 14.4 & 4.4. & 7.7 & 29 & 1.2 \\
\hline 43 & 53.8 & 26 & 12.9 & 14.1 & 4.4 & 7.6 & 28 & 1. \\
\hline
\end{tabular}


DH -3

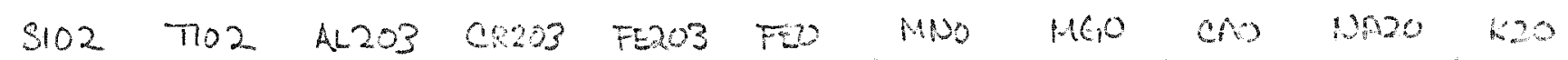

44

45

46

47

48

49

50

51

52

53

54

55

56

औ)

38

59

60

1.6

61

62

63

64

65

66

$157 \times 52.6$

$67 \quad 55.0 \quad 2.4 \quad 12.1$

$68 \quad 56.4 \quad 1.7,12.6$

$15 \% \quad 53.1 \quad 2.8: 13.2$

$69 \times 57.7 \quad 1.7 \quad 12.2$

$159-\frac{55.1}{56.2}-\frac{20}{1.6} \frac{16.3}{12.7}$

$\begin{array}{llll}70 & 56.2 & 1.6 & 12.7 \\ 71 & 564 & 1.6 & 12.1\end{array}$

$\begin{array}{llll}72 & 55.9 & 1.6 & 12.9\end{array}$

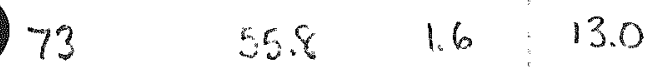

$\begin{array}{lll:l}74 & 36.4 & 1.7 & 12.5\end{array}$

$\begin{array}{lll:l}75 & 567 & 1.7 & 12.7\end{array}$

$\begin{array}{llll:l}76 & 4 & 57.4 & 1.7 & 12.4\end{array}$

$\begin{array}{lll:l}77 & 57.6 & 1.6 & 12.4\end{array}$
14.1

14.1

13.7

149

14.1

13.9

13.7

14.2

14.4

13.8

14.5

14.8

14.3

14.1

14.4

11.5

14.4

15.3

14.4

14.0

14.6

14.8

15.7

15.7

14.9

12.5

15.0

14.5

$10 \%$

13.1

13.5

12.5

13,2

13.4

13.0

12.8

12.6
$4.4: 79: 29: 4$

\begin{tabular}{l|l:l|l}
3.8 & 7.7 & 3.0 & 1.2
\end{tabular}

\begin{tabular}{l|l:l|l}
4.2 & 8.0 & 2.8 & 1.4
\end{tabular}

\begin{tabular}{l|l|l}
44 & 74.25
\end{tabular}

\begin{tabular}{l|l|l}
4.8 & 8.0 & 26
\end{tabular}

\begin{tabular}{l|l|l}
4.7 & 8.1 & 2.7
\end{tabular}

$4.7: 8.0: 2.6$

$4.4: 7.6: 3.7$

$45: 7.9: 28$

$4.2 \div 7.9$

2.9

$5.2 \quad 8.1$

25

$4.9 \quad 7.9$

2.7

7.8

2.7

2.8

$4.6 \quad 7.6$

$3.7 \quad 77$

28

44

7.

4.37 .5

4.6

77

4.3

37

4.

4.2

4.3

4.3

7.6

7.6

29

28

20

2.7

2.0

2.7

75

19

25

7.3

7,3

2.3

$\begin{array}{lll}38 & 80 & 28\end{array}$

$5.1 \quad 7.8$

30

$4.7 \quad 7.3$

2.8

2.8

3.7

2.5

3.8

4.5

7.0

3.1

4.7

7.9

3.1

76

3.0

$5.1 \quad 8.2$

$5.2 \quad 7.9$

2.3

2.6

4.8

7.6

26

$4.7 \quad 7.6: 2.8$

4.5

7.3

27

$47 \quad 72 \quad 26$
1.2

1.2

13

1.1

1.2

1.2

14

10

1.3

1.3

12

12

1.2

1.1

10

1.1

10

1.1

1.0

1.1

9


DH -3

$\$ 102 \pi 702$ AL2O3 CR2O3 FE2O3 FEO MNO MGO CAO NA2O K2O

\begin{tabular}{l|l|l|l}
78 & 57.2 & 1.7 & 12.7 \\
79 & 574 & 1.6 & 12.7 \\
80 & 57.7 & 16 & 12.5 \\
81 & 58.0 & 1.6 & 126 \\
82 & 55.9 & 1.8 & 12.9 \\
161 & 54.1 & 1.9 & 14.0 \\
83 & 54.4 & 1.6 & 12.9 \\
84 & 549 & 1.5 & 12.7
\end{tabular}

\begin{tabular}{ll|l|l}
85 & 55.8 & 1.6 & 12.6
\end{tabular}

$86 H^{\prime} 55.6: 17,130$

$87 \cdot 55.8 \quad 17 \quad 129$

$88 \quad 55.4 ; 1.7 \quad 131$

$\begin{array}{llll}89 & 55.0 & 1.8 & 12.9\end{array}$

$92 \quad 54.3,17,13.2$

$93 \quad 53.5 .18 \quad 139$

$94 \quad 55.7: 1.7,131$

$95 \quad 54.4,1.8,13.6$

$96 \quad 537: 1.8 \quad 134$

$97 \quad 54.1,17 \quad 13.5$

\begin{tabular}{ll|ll}
98 & 53.7 & 19 & 139
\end{tabular}

$\begin{array}{ll:lll}99 & 55.7 & 1.7 & 13.3\end{array}$

$\begin{array}{ll:ll}100 & 547 & 1.8 & 13.8\end{array}$

$101-51.8,1.9,14.7$

$102 \quad 54.1,1.8 \quad 13.6$

$\begin{array}{lllll}103 & 535 & 1.8 & 13.9\end{array}$

$104: 529 \quad 1.8 \quad 13.9$

$\begin{array}{lll}105 & 53.9 & 1.9 \\ 106 & 14.3\end{array}$

$\begin{array}{llll}106 & 56.0 & 18 & 133\end{array}$

$108 \quad \frac{560}{58.6} \quad 2.0 \quad \frac{18}{12.7}$

$109 \quad 58.9 \cdot 1.9 \quad 125$

$110 \quad 57.1,2.0 \quad 13.2$

$111 \quad 56.5 ; 2.112 .9$

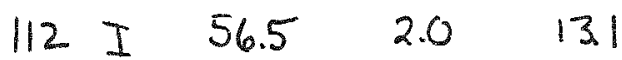

$\begin{array}{llll}113 & 56.1 & 2.6 & 130\end{array}$

$\begin{array}{llll}114 & 55.3 & 22 & 13.3\end{array}$

$\begin{array}{llll}115 & 55.1 & 22 & 13.3\end{array}$

$116,56.1,2.0,13.5$

\begin{tabular}{l|l|l|l}
117 & 55.8 & 2.0 & 134
\end{tabular}

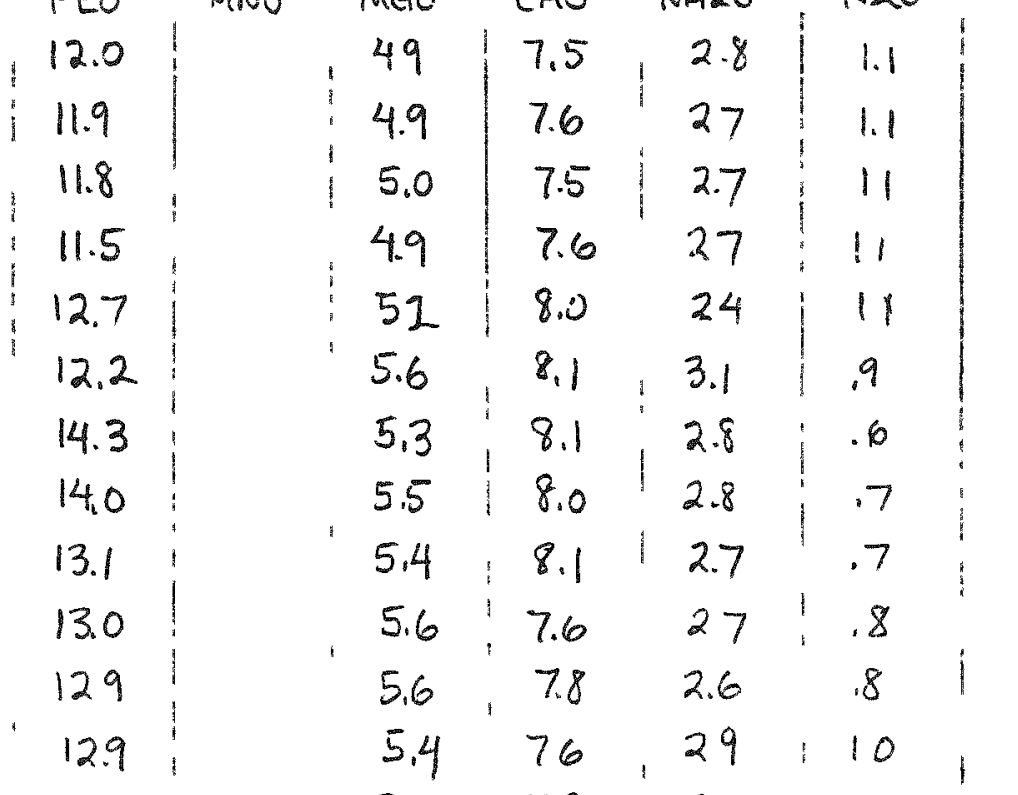

$13.5, \quad 5.7 \quad 7.9,2.7, .7$

\begin{tabular}{lll|l|l}
13.2 & 5.5 & 8.3 & 29 & .9
\end{tabular}

$\begin{array}{lllll}13.0: & 5.5 & 8.5 & 27 & 1.0\end{array}$

12.6

13.1

12.9

133

13.0

12.3

12.2

12.8

12.5

13.2

13.3

12.8

122

12.0

12.3

118

13.1

12.7

13.4

13.4

14.1

13.3

13.1
$5.1,83 \quad 2.9$

$5.2 \quad 8.2,3.0$

$5.4 \quad 91$

27

$\begin{array}{lll}5.5 & 8.6,27\end{array}$

$5.4,8.5,30$

$5.3,8.3,29$

$\begin{array}{lll}5.5 & 8.1 & 27\end{array}$

$\begin{array}{lll}6.7 & 85 & 2.8\end{array}$

$6.2 \quad 8.1$

3.0

$\begin{array}{lll}5.8 & 7.9 & 2.8\end{array}$

$5.4 \quad 8.7$

2.8

12

$\begin{array}{lll}5.2 & 8.1 & 3.0\end{array}$

49

3.4

63

3.4

6.9

$3.7 \quad 7.0 \quad 2.8$

$3.6 \quad 7.0,3.1$

$3.2 \quad 21$

$3.7 \quad 6.8$

3.2

$3.8 \quad 6.9$

3.5

1.4

$3.9 \quad 6.9$

$\begin{array}{llll}3.8 & 69 & 3.2 & 14\end{array}$

1.5

$\begin{array}{lll}3.7 & 6.8 & 3.4\end{array}$

13

$3.9 \quad 7.0 \quad 34$ 
$\$ 102$ TIO2 AL2O3 CR2O3 FE203 FEO MNO MGO CAO NA2O K2O

119

120

$: 56.02 .1,13.7$,

12.7

\begin{tabular}{l|l}
3.8 & 7.0 \\
3.5 & 6.8
\end{tabular}

3.3

$\begin{array}{llll}57.2 & 2.1 & 1 & 12.9\end{array}$

$\begin{array}{lllll}136 & 36 & 67 & 31 & 1.5\end{array}$

122

57.3

12.0

5.4

8.5

129

53.9

2.0

12.7

52.5

2.0

14.5

$136 \mathrm{~J} 54.0$

$1.7 \quad 139$

13.3

134

12.2

5.0

8.8

3.1

123

$55.1 \cdot 1.8$

12.7

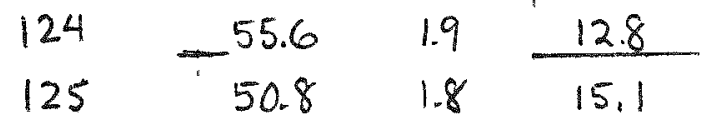

12.4

\begin{tabular}{l|l}
5.7 & 8.5
\end{tabular}

$2.9 ; .9$

$\begin{array}{lllll}126 & k & 52.2 & 1.8 & 14.6\end{array}$

12.4

123

11.8

$\begin{array}{llll}128 & 53.1 & 1.8 & 14.2\end{array}$

11.5

$127 \quad \frac{53.2}{56.1} 1.7 \frac{14.3}{139}$

$\frac{11.5}{13.4}$

$5.8: 80$

3.1

3.2

\begin{tabular}{l|l}
5.3 & 7.9 \\
\hline 6.6 & 9.8
\end{tabular}

3.1

3.2

.9

\begin{tabular}{l|l}
6.6 & 9.8 \\
6.4 & 94
\end{tabular}

33

6.4

9.0

33

\begin{tabular}{l:l}
6.0 & 9.3 \\
\hline 4.5 & 5.4
\end{tabular}

3.2

1.0

1.0

.4

9.3

2.8

.7 
DH -4

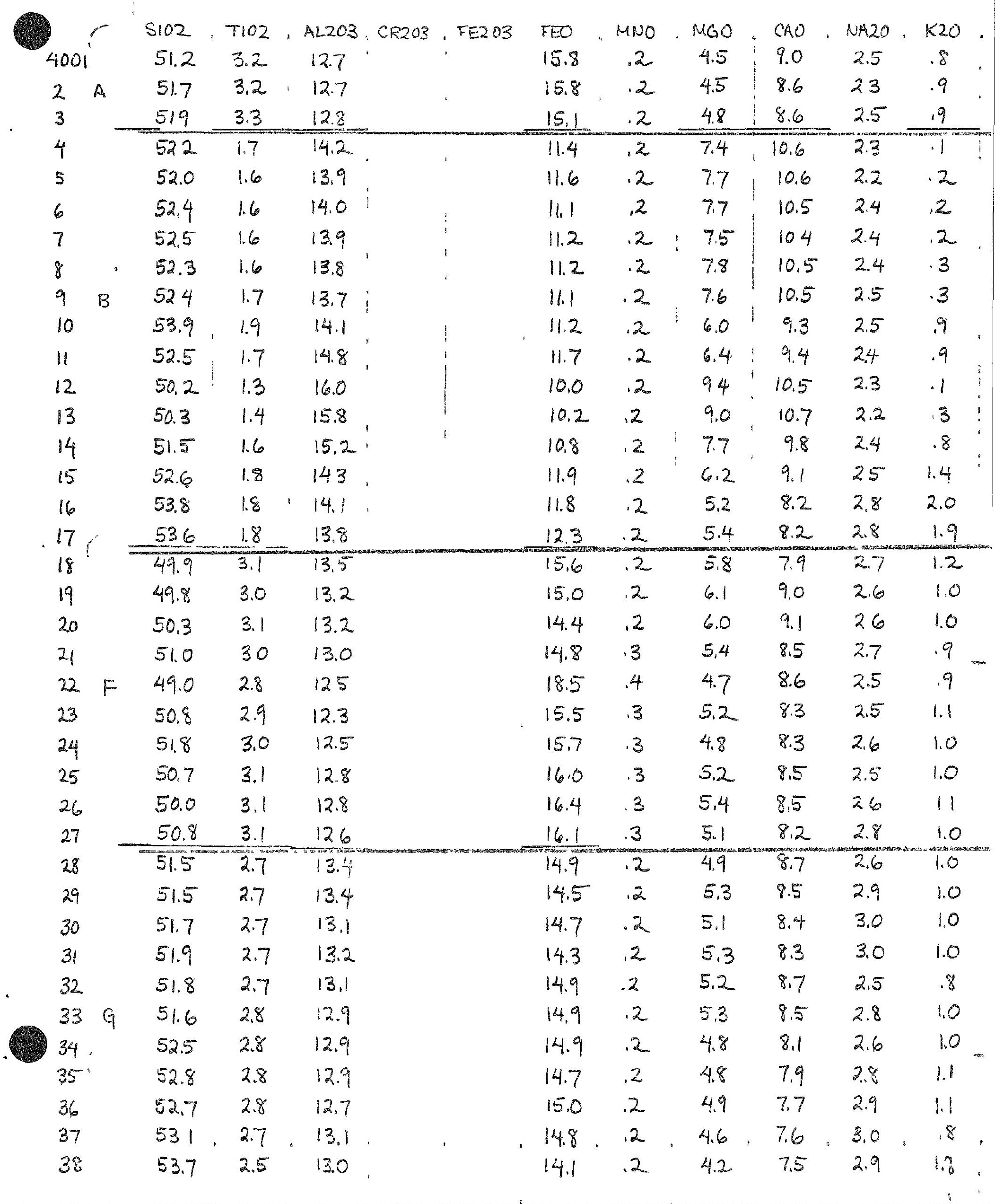




\begin{tabular}{|c|c|c|c|c|c|c|c|c|c|c|c|c|}
\hline & & 8102 & 7102 & , AL203 & CR2O3 & FE203 & FEO & MNO & $M G O$ & CAO & 1.920 & $K 20:$ \\
\hline 39 & & 52.9 & 2.6 & 12.0 & & & 15.5 & .2 & 4.4 & 7.3 & 2.9 & 1.1 \\
\hline 40 & & 54.5 & 2.6 & 13.0 & & & 13.7 & .2 & 3.9 & 72 & 3.1 & 1.7 \\
\hline 41 & & 53.6 & 2.6 & 13.0 & & & 14.8 & .2 & 4.6 & 7.2 & 2.9 & 1.0 \\
\hline 42 & & 53.5 & 2.8 & 13.3 & & & 14.4 & 2 & 4.3 & 7.8 & 2.7 & 1.0 \\
\hline 43 & & 52.2 & 2.7 & 13.5 & & & 15.3 & 2 & 4.8 & 7.8 & 2.8 & .7 \\
\hline 44 & & 52.5 & 3.0 & 13.2 & & & 15.1 & .2 & 4.7 & 7.7 & 2.8 & .8 \\
\hline 45 & & 52.1 & 2.9 & 13.1 & & & 16.2 & .2 & 4.2 & 7.5 & 2.9 & .7 \\
\hline 46 & & 53.5 & 2.7 & 12.6 & & & 15.6 & .2 & 4.9 & 7.1 & 25 & .7 \\
\hline 47 & & 53.2 & 2.7 & 13,2 & & & 14.8 & .2 & 4.6 & 7.3 & 2.9 & 1.0 \\
\hline 48 & $G$ & 53,3 & 2.8 & 12.8 & & & 15.1 & .2 & 4.7 & 72 & 3.0 & .9 \\
\hline 49 & & 52.8 & 2.9 & 13.5 & & & 14.8 & .2 & 4.6 & 7.2 & 3.2 & .7 \\
\hline 50 & & 53.2 & 2.8 & 13.3 & & & 14.9 & .2 & 4.7 & 7.1 & 3.1 & .7 \\
\hline 51 & & 51.3 & 2.7 & 13.3 & & & 15.4 & .3 & 4.9 & 8.5 & 2.8 & .7 \\
\hline 52 & & 53.3 & 2.8 & 13.2 & & 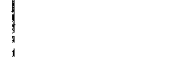 & 15.0 & .2 & 4.8 & 7.3 & 2.8 & .5 \\
\hline 53 & & 53.4 & 2.8 & 132 & & & 15.2 & .3 & 4.6 & 7.0 & 2.8 & .7 \\
\hline 54 & & 53.2 & 2.7 & 13.3 & & & 14.9 & .2 & 4.8 & 7.1 & 3.0 & .7 \\
\hline 55 & & 52.8 & 2.6 & 13.0 & & & 15.6 & 2 & 5.0 & 72 & 3.0 & .6 \\
\hline 56 & & 52.1 & 2.8 & 128 & & & 14.5 & .2 & 4.1 & 10.0 & 2.6 & 1.0 \\
\hline 57 & & 52.9 & 2.8 & 13.0 & & & 14.3 & .2 & 4.7 & 8.2 & 2.8 & 1.1 \\
\hline 58 & & 52.6 & 2.7 & 12.8 & & & 15.2 & .3 & 4.6 & 8.0 & 2.8 & 1.0 \\
\hline 59 & & 52.6 & 2.7 & 13.3 & & & 14.8 & .3 & 4.2 & 8.1 & 2.8 & 1.2 \\
\hline 60 & & 52.5 & 2.6 & 13.2 & & & 14.6 & .2 & 4.6 & 8.0 & 3.1 & 1.2 \\
\hline 61 & & 526 & 2.7 & 13.1 & & & 14.7 & 3 & 4.5 & 8.1 & 3.0 & .9 \\
\hline 62 & & 52.1 & 2.7 & 13.0 & & & 15.0 & .3 & 4.7 & 8.2 & 3.1 & .9 \\
\hline 63 & & 52.5 & 2.6 & 129 & & & 15.1 & 2 & 4.8 & 77 & 31 & 1.0 \\
\hline $\begin{array}{l}64 \\
65\end{array}$ & - & 542 & 1.6 & 14.4 & & & $11.8^{\circ}$ & 2 & 5.6 & 85 & 3.0 & .7 \\
\hline 66 & & 54.2 & 17 & $14:$ & & & 11.6 & .2 & 5.1 & 8.1 & 3.0 & .9 \\
\hline 67 & & 54.5 & 1.7 & 14.3 & & & 11.3 & .2 & 5.5 & 8.5 & 3.1 & .8 \\
\hline 68 & & 54.5 & 1.8 & 14.5 & & & 11.5 & .2 & 5.4 & 8.5 & 30 & .6 \\
\hline 69 & & 54.3 & 1.7 & 13.9 & & & 12.1 & .2 & 54 & 8.7 & 3.1 & .5 \\
\hline 70 & $H$ & 53.0 & 1.7 & 14.2 & & & 12.1 & .2 & 5.6 & 9.9 & 3.0 & .3 \\
\hline 71 & & 53.2 & 17 & 14.5 & & & 12.2 & .2 & 5,9 & 8.8 & 30 & 5 \\
\hline 72 & & 54.5 & 1.8 & 14.3 & & & 11.8 & .2 & 5.3 & 8.5 & 3.0 & .5 \\
\hline 73 & • & 53.3 & 1.8 & 14.1 & & & 12.4 & .2 & 5.8 & 8.1 & 3.1 & 1.4 \\
\hline 74 & & 54.2 & 1.7 & 14.2 & & & 11.9 & .2 & 54 & 8.4 & 2.9 & 1.0 \\
\hline 75 & & 533 & 18 & 14.1 & & , & 12.4 & .2 & 5,8 & 8.1 & 3.1 & 11 \\
\hline 76 & & 55.2 & 1.7 & 13.5 & & i & 11.7 & .2 & 5.4 & 8.3 & 3.0 & 1.0 \\
\hline
\end{tabular}


DH -4

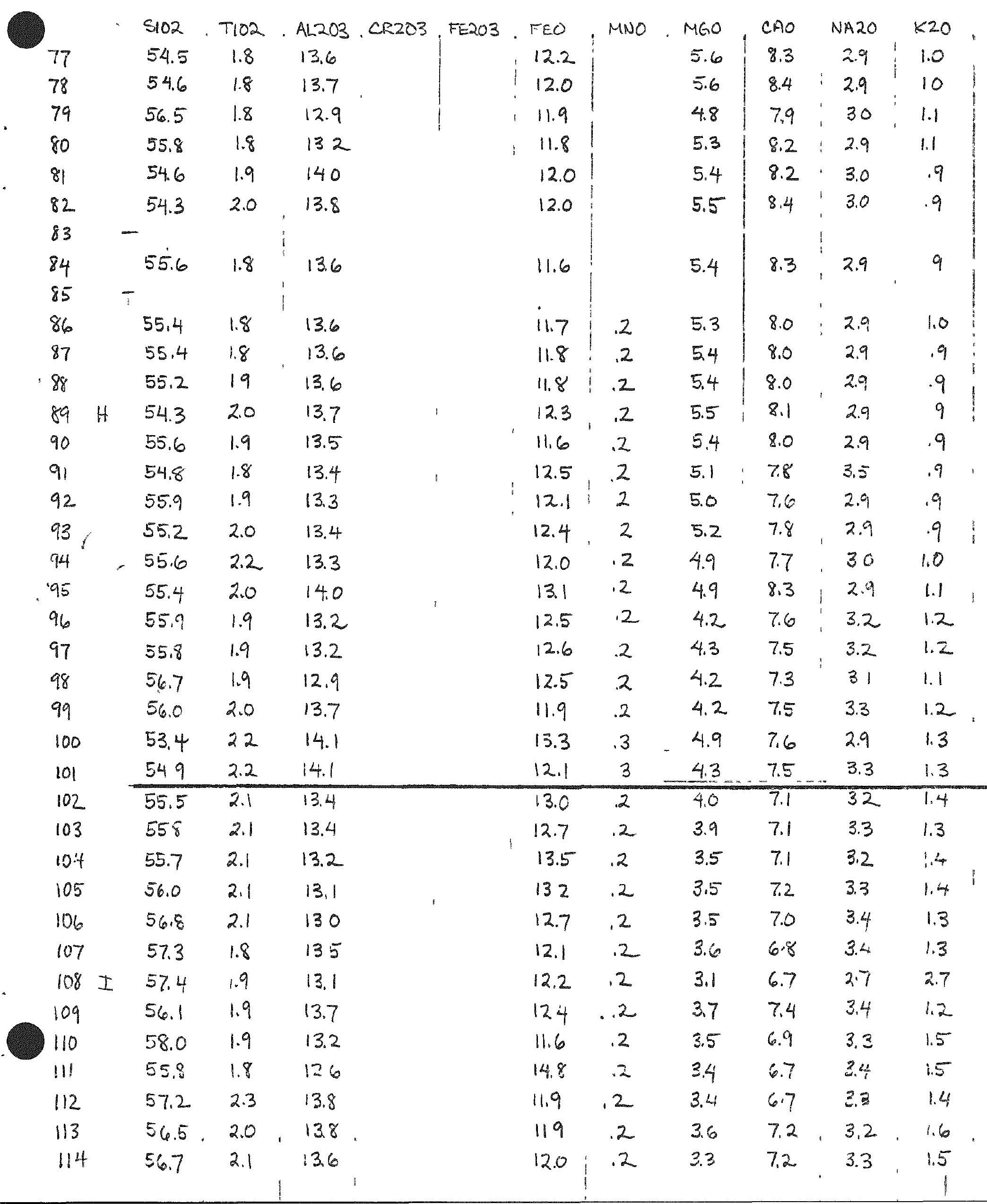




\section{DH -4}

S102, T102, AL203,CR203, FE203, FO, MNO, MGO, CAO, NA2O, K20

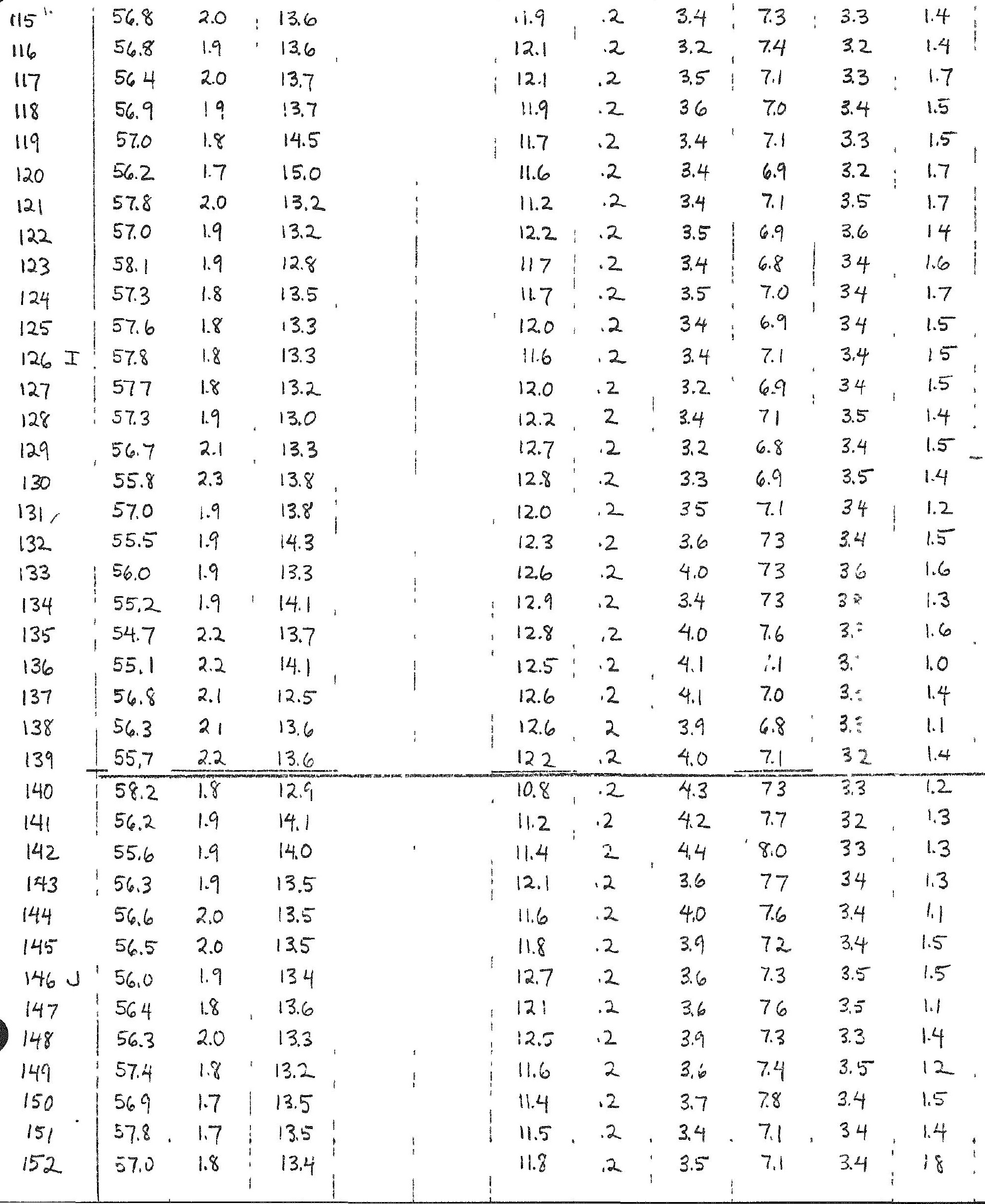


DH -4

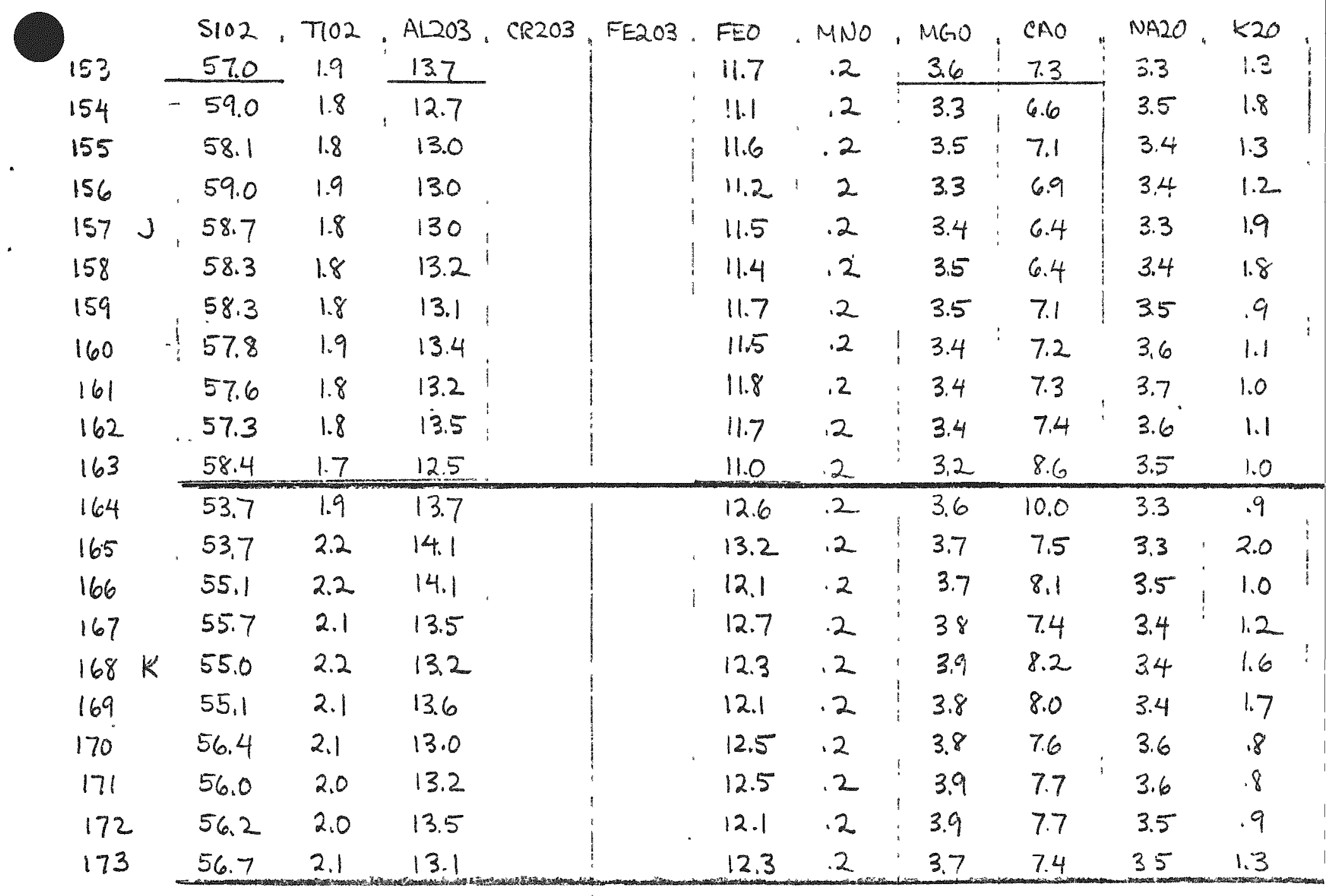


DH -5

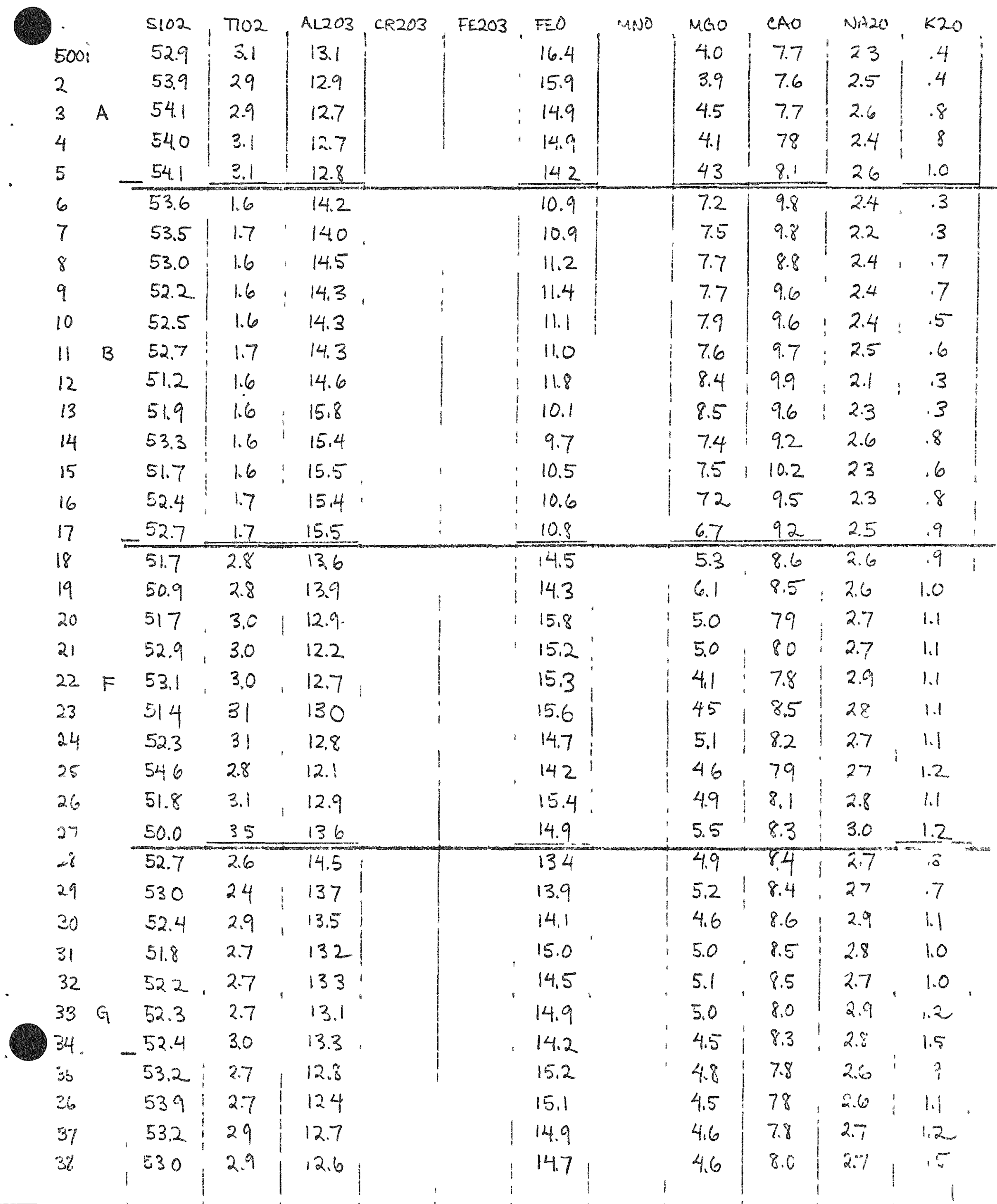


DH. 5

$\$ 102, T 102, A L 203$, CR203, FE203

$53.1 \quad 2.5$

40

51.8

3.0

41 $53.9 \div 2.6$

42

540

29

43

44

45

$54.3 \quad 2.6$

$53.9 \quad 2.5$

$54.0 \quad 2.6$

$53.4 \quad 2.9$

46

$47 G: 53.1 \quad 3.2$

48

$53.2 \quad 3.0$

49

$51.5 \quad 2.9$

13.2

13.3

12.8

12.7

12.6

12.8

12.9

12.9

13.2

13.1

50

$\begin{array}{ll}55.0 & 2.7\end{array}$

12.9

$\begin{array}{ll}53.5 & 2.7\end{array}$

52

53

$53.4 \quad 2.8$

$53.5 \quad 3.0$

12.5

12.9

12.2

54

$52.8 \quad 2.9$

12.8

55

$52.2 \quad 3.0$

133

56

52.9

3.1

12.8

$\begin{array}{llll}57 & 51.0 & 3.0 & 14.0\end{array}$

$\begin{array}{llll}58 & 52.5 & 3.1 & 12.3\end{array}$

$\begin{array}{llll}59 & 51.7 & 3.2 & 13.1\end{array}$

$\begin{array}{llll}60 & 51.7 & 3.0 & 13.2\end{array}$

$61 \quad 524 \quad 3.0 \cdot 13.3$

$\begin{array}{llll}62 & 54.5 & 2.8 & 12.5\end{array}$

$63-52.9 \quad 2.9-130$

$64 \quad 54.7 \cdot 1.6,13.8$

$\begin{array}{llll}65 & 55.4 & 1.7,13.6\end{array}$

$66 \quad 55.3 \cdot 1.6 \quad 14.2$

$\begin{array}{lllll}67 & 55.2 & 1.6 & 14.3\end{array}$

$\begin{array}{llll}68 & 555 & 1.5 & 14.4\end{array}$

$\begin{array}{lll:l}69 H & 55.4 & 1.5 & 14.3\end{array}$

$70 \quad 55.0 \cdot 1.8 \quad 14.2$

$\begin{array}{llll}71 & 54.9 & 18 & 139\end{array}$

$\begin{array}{llll}12 & 54.6 \quad 1.6 & 1 & 14.2\end{array}$

$\begin{array}{llll}73^{\circ} & 54.9 & 16 & 13.9\end{array}$

$\begin{array}{llll}7.1 & 542 & 1.9 & 140\end{array}$

$75: 54.1,1.7,13.9$

76

13.7
13.1

\begin{tabular}{c|c|} 
FED & MNO \\
14.5 & .2 \\
14.8 & .2 \\
14.5 & .2 \\
14.2 & .2 \\
14.2 & 2
\end{tabular}

\begin{tabular}{l|l:}
14.2 & .2
\end{tabular}

\begin{tabular}{l|l|l}
14.4 & .2 & 4.6
\end{tabular}

$14.2, .2$

\begin{tabular}{l|l}
14.3 & .2
\end{tabular}

14.1

2

$14.0: .2$

$14.8: .2$

$12.0 ; .3$

$14.6, .3$

$14.5,3$

$14.9 \cdot 3$

$14.6 \quad .2$

$14.8, .2$

$14.7 \quad .2$

$15.5,2$

$14.9 \quad .2$

$14.7 \quad 2$

14.4 .2

14.4 .2

$13.6 \quad .2$

14.2 .2

118.2

$11.4: .2$

10.9

11.0 .2

$10.7, .2$

$11.3 \quad .2$

$11.2,2$

$11.4 \quad 2$

$11.4 \quad .2$

113.2

11.7

11.8

11.6

4.5

4.7

4.5

4.5

4.5

4.6

4.7

4.8

4.8

5.1

4.6

4.3

4.1

45

4.3

4.2

4.1

44

4.4

4.4

4.4

52

4.8

50

4.9

4.8

48

48

5.3

5.8
NGO, CAO, NA2O, K2O

7.4

7.4

7.5

7.6

7.7

7.6

8.2

8.4

84

8.2

8.1

8.2

8.5

8.

7.9

8.0

8.6

$4.9 \div 8.6$

8.3

$4.1,8.0$

$42 \quad 8.2$

8.3

8.8

86

8.6

$4.8,8.4$

8.9

8.7

$5.1 \div 8.7$

$5.1 \quad 8.6$

9.0 \begin{tabular}{l|ll}
5.9 & 92 & 3.0
\end{tabular}
2.8

2.8

29

2.8

2.8

15

2.6

1.2

2.6

1.3

2.6

2.7

1.3

3.0

1.3

3.1

2.7

1.1

2.8

2.7

29

2.8

1.0

2.9

1.2

$2.9 \quad 1.0$

3.1

1.3

3.0

1.2

3.1

1.1

30

1.0

3.1

3.1

1.2

2.9

3.2

3.0

3.1

3.2

1.0

16

1.3

.9

1.0

3.1

10

3.0

1.1

3.1

12

3.1

3.3

1.0

1.2

10

.9 
$5102, T 102, A 1203, C R 203, E 203$ FEO MNO MGO CAO NJ420 K2O

77 $54.0,1.8,136$

78 $53.8,18,13.9$

80

$$
51.8
$$

81

$530 \quad 19$

13.4

83

126

$\frac{528}{57.1} 19$

13.7

111.6

127

$57.5 \quad 1.9$

139

128

$\begin{array}{ll}54.2 & 19\end{array}$

13.6

129

55.7

13.7

13.7

$\begin{array}{llll}130 & 56.0 & 2.0 & 144\end{array}$

$\begin{array}{llll}134 & 56.1 & 20 & 13.3\end{array}$

$\begin{array}{l:lll}135 & 56.7 & 2.1 & 13.3\end{array}$

\begin{tabular}{l|lll}
136 & 56.4 & 2.2 & 134
\end{tabular}

\begin{tabular}{l|lll}
137 & 567 & 2.1 & 134.
\end{tabular}

$138 I, 56.0,20 \quad 135$

$139 \quad 56.2 \quad 20: 13.6$

$140,55.6 \quad 21,134$

$\begin{array}{llll}141 & 55.1 & 2.1 & 137\end{array}$

$142,55.2,21,13.7$

$143: 55.4 \quad 19 \quad 14.0$

$144,53.1,22,141$

\begin{tabular}{ll|l|l}
145 & 562 & 2.0 & 3.5
\end{tabular}

$146,558,2.1,13.7$

$147 \quad 55.9 \quad 2.0,135$

$\begin{array}{llll}148 & 55.4 & 21 & 134\end{array}$

\begin{tabular}{l|l|l|l}
149 & 56.0 & 21 & 13.6
\end{tabular}

11,5

119

$5.9 ; 92 \div 2.9: 1.0$

\begin{tabular}{l|ll}
5.9 & 90 & 28
\end{tabular}

\begin{tabular}{l|ll}
5.6 & 8.8 & 29
\end{tabular}

$\begin{array}{llll}5.8 & 89 & 30 & 18\end{array}$

119

60

3.7

9 
DH -5

S102, T102, AL203, IR203, FE203 =EO, MNO, MGO, CFO, IAF2, '<20

$162,57.1,2.0 \quad 135$

$163,56.4,22 \quad 135$

$164: 56.6 \cdot 24 \quad 136$

$165: 568 \quad 22: 33$

$166,561 \quad 24 \quad 13.3$

$\begin{array}{llll}167 & 560 & 23 & 13.7\end{array}$

$168 \quad 55.2 \quad 23 \quad 136$

$\begin{array}{llll}169 & 55.5 & 23 & 13.1\end{array}$

$\begin{array}{llll}170 & 55.8 & 22 & 132\end{array}$

$\begin{array}{llll}171 & 53.6 & 2.3 & 134\end{array}$

$\begin{array}{llll}174 & 542 & 23 & 13.9\end{array}$

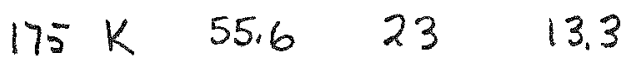

$181 \quad 54.6 \quad 23 \quad 13 ?$

$\begin{array}{llll}182 & 55.2 & 22 & 13.4\end{array}$

$\begin{array}{llll}183 & 569 & 2.2 & 12.6\end{array}$

$\begin{array}{llll}184 & 55.5 & 23 & 13.3\end{array}$

$\begin{array}{llll}185 & 545 & 2.4 & 135\end{array}$

$\begin{array}{llll}186 & 562 & 22 & 13.6\end{array}$

$\begin{array}{llll}187 & 55.9 & 22 & 135\end{array}$

$\begin{array}{llll}188 & 55.6 & 23 & 136\end{array}$

$\begin{array}{llll}189 & 55.8 & 22 & 133\end{array}$

$\begin{array}{llll}190 & 55.3 & 2.3 & 13.3\end{array}$

$\begin{array}{llll}191 & 55.7 & 24 & 13.4\end{array}$

$\begin{array}{llll}192 & 56.0 & 2.3 & 13.3\end{array}$

$\begin{array}{llll}193 & 546 & 2.4 & 13.5\end{array}$

$\begin{array}{llll}194 & 55.8 & 2.2 & 132\end{array}$

$\begin{array}{llll}195 & 56.1 & 22 & 13.3\end{array}$

$\begin{array}{llll}196 & 562 & 2.2 & 134\end{array}$

$\begin{array}{llll}197 & 55.8 & 23 & 13.5\end{array}$

$\begin{array}{llll}198 & 561 & 22 & 133\end{array}$

$\begin{array}{llll}199 & -55.1 & 22 & 13.4\end{array}$

$201-548 \quad 23 \quad 137$

$\begin{array}{llll}202 & 55.5 & 21 & 137\end{array}$

$\begin{array}{llll}202 & 55.7 & 23 & 126\end{array}$

isir $\quad \begin{array}{lll}526 & 24 & 143\end{array}$

$\left|\begin{array}{c}121 \\ 123\end{array}\right|$

12.2

12.1

126

122

127

13.2

12.7

12.2

12.4

13.0

125

125

122

125

12.9

126

125

127

12.5

12.0

128

12.6

132

131

12.9

12.8

13.1

125

133

12.8

124

12.9

35

3.8

3.7

3.8

37

$\begin{array}{llll}3.7 & 6.7 & 33 & 15 \\ 3.8 & 72 & 33 & 12 \\ 37 & 70 & 32 & 1.8 \\ 3.7 & 69 & 34 & 18 \\ 40 & 67 & 33 & 22 \\ 43 & 73 & 35 & 19 \\ 40 & 71 & 34 & 1.6 \\ 4.0 & 75 & 33 & 3.5 \\ 44 & 79 & 31 & 18 \\ 4.2 & 73 & 32 & 11 \\ 43 & 8.0 & 32 & 19 \\ 45 & 73 & 34 & 1.6 \\ 39 & 72 & 33 & 17 \\ 4.0 & 74 & 34 & 1.6 \\ 3.8 & 7.2 & 29 & 2.9 \\ 36 & 71 & 33 & 14 \\ 39 & 7.2 & 33 & 1.5 \\ 37 & 71 & 33 & 18 \\ 3.5 & 6.6 & 34 & 27 \\ 34 & 69 & 3.2 & 30 \\ 35 & 68 & 33 & 22 \\ 3.5 & 6.6 & 35 & 2.1 \\ 36 & 7.0 & 34 & 22 \\ 34 & 6.8 & 32 & 20 \\ 35 & 67 & 34 & 1.9 \\ 35 & 67 & 3.3 & 1.8 \\ 3.5 & 67 & 33 & 18 \\ 3.5 & 6.6 & 32 . & 27 \\ 35 & 66 & 33 & 2.7\end{array}$

$\begin{array}{llll}3.7 & 6.7 & 33 & 15 \\ 3.8 & 72 & 33 & 12 \\ 37 & 70 & 32 & 1.8 \\ 3.7 & 69 & 34 & 18 \\ 40 & 67 & 33 & 22 \\ 43 & 73 & 35 & 19 \\ 40 & 71 & 34 & 1.6 \\ 4.0 & 75 & 33 & 3.5 \\ 44 & 79 & 31 & 18 \\ 4.2 & 73 & 32 & 11 \\ 43 & 8.0 & 32 & 19 \\ 45 & 73 & 34 & 1.6 \\ 39 & 72 & 33 & 17 \\ 4.0 & 74 & 34 & 1.6 \\ 3.8 & 7.2 & 29 & 2.9 \\ 36 & 71 & 33 & 14 \\ 39 & 7.2 & 33 & 1.5 \\ 37 & 71 & 33 & 18 \\ 3.5 & 6.6 & 34 & 27 \\ 34 & 69 & 3.2 & 30 \\ 35 & 68 & 33 & 22 \\ 3.5 & 6.6 & 35 & 2.1 \\ 36 & 7.0 & 34 & 23 \\ 34 & 6.8 & 34 & 20 \\ 35 & 67 & 34 & 1.9 \\ 35 & 67 & 3.3 & 1.8 \\ 3.5 & 67 & 33 & 18 \\ 3.5 & 6.6 & 32 . & 27 \\ 35 & 66 & 33 & 2.7\end{array}$

$\begin{array}{llll}3.7 & 6.7 & 33 & 15 \\ 3.8 & 72 & 33 & 12 \\ 37 & 70 & 32 & 1.8 \\ 3.7 & 69 & 34 & 18 \\ 40 & 67 & 33 & 22 \\ 43 & 73 & 35 & 19 \\ 4.0 & 71 & 34 & 1.6 \\ 4.0 & 75 & 33 & 3.5 \\ 44 & 79 & 31 & 18 \\ 4.2 & 73 & 32 & 11 \\ 43 & 8.0 & 32 & 19 \\ 45 & 73 & 34 & 1.6 \\ 39 & 72 & 33 & 17 \\ 4.0 & 74 & 34 & 1.6 \\ 3.8 & 7.2 & 29 & 2.9 \\ 36 & 71 & 33 & 14 \\ 39 & 7.2 & 33 & 1.5 \\ 37 & 71 & 33 & 18 \\ 3.5 & 6.6 & 34 & 27 \\ 34 & 69 & 3.2 & 30 \\ 35 & 68 & 33 & 22 \\ 3.5 & 6.6 & 35 & 2.1 \\ 36 & 7.0 & 34 & 23 \\ 34 & 6.8 & 34 & 20 \\ 35 & 67 & 34 & 1.9 \\ 35 & 67 & 3.3 & 1.8 \\ 3.5 & 67 & 33 & 18 \\ 3.5 & 6.6 & 32 . & 27 \\ 35 & 66 & 33 & 2.7\end{array}$

40

40

44

4.2

43

45

39

\section{4.}

3.

36

3

37

3.5

\section{3}

3

\section{3}

\section{1}

34

35

35

3.5

3.5

3 
APPENDIX B

Data in parts per million 
DH-I

$S C$ CR CO BA LA SM EU, TB HF TA TH $20 \quad 60 \quad 580$

\begin{tabular}{rrrrr}
$-A$ & 1 & 20 & 60 & 580 \\
2 & 60 & 40 & 650 \\
\hline 3 & 40 & 440 & 825 \\
4 & 40 & 440 & 570 \\
5 & 40 & $<40$ & 525 \\
6 & 120 & 440 & 410 \\
& & & \\
7 & 80 & 40 & 350 \\
8 & 100 & 440 & 400
\end{tabular}

B

$9 \quad 120 \quad 40 \quad 340$

$10 \quad 100 \quad 440 \quad 370$

$\because \quad 100 \quad 40 \quad 370$

12. $\quad 100 \quad 40 \quad 375$

\begin{tabular}{rrrr}
13 & 120 & 40 & 350 \\
14 & 80 & 440 & 375 \\
\hline 15 & 60 & 440 & 825
\end{tabular}

$16 \quad 60<40 \quad 725$

$17 \quad 20 \quad 440 \quad 750$

$c$

$18 \quad 40 \quad 40 \quad 725$

$19 \quad 20 \quad 40 \quad 650$

\begin{tabular}{llll}
20 & 40 & $<40$ & 710 \\
\hline 21 & 20 & $<40$ & 500
\end{tabular}

D $22 \quad 180 \quad 440 \quad 540$

\begin{tabular}{lllll} 
& 23 & 160 & $\angle 40$ & 615 \\
\hline & 24 & $\angle 20$ & $<40$ & 3800 \\
$E$ & 25 & $<20$ & $<40$ & 3405
\end{tabular}


$\mathrm{DH}-1$

SC CR CO BA LA SM EU TB HF TA TH

$\begin{array}{cccc}26 & <20 & \angle 40 & 3300 \\ 27 & <20 & 40 & 3190 \\ 28 & 20 & 440 & 2740\end{array}$

$\begin{array}{lllll}29 & 20 & 440 & 2830 \\ 30 & 20 & 440 & 2850\end{array}$

$31 \quad 20 \quad 440 \quad 2700$

\begin{tabular}{lllll}
32 & 20 & 440 & 1710 \\
\hline (1) 33 & $\angle 20$ & 440 & 600 \\
34 & 20 & $\angle 40$ & 790 \\
\hline
\end{tabular}

\begin{tabular}{rrrrr}
35 & 80 & 440 & 630 \\
(2) 36 & 80 & 440 & 690 \\
37 & 80 & 40 & 660 \\
& & & \\
38 & 100 & 440 & 680 \\
39 & 100 & 40 & 580 \\
40 & 100 & 40 & 595 \\
\hline
\end{tabular}

$\begin{array}{llll}41 & 80 & 40 & 595 \\ 42 & <20 & 440 & 615 \\ 43 & <20 & 40 & 615 \\ \text { (3) } 44 & 40 & 440 & 665 \\ 45 & 40 & 440 & 820 \\ 46 & 40 & 440 & 730 \\ 47 & 46 & 440 & 640 \\ 48 & 40 & 440 & 735\end{array}$


DH-2

SG CR CO BA LA SM ELA TB HF TA TH

$\begin{array}{llrr}49 & 190 & -84 & -920 \\ 50 & 320 & 74 & 840\end{array}$

A

$51 \quad 295 \quad 55 \quad 680$

\begin{tabular}{lllll}
\hline & 52 & 460 & 35 & 635 \\
& 53 & 200 & 61 & 445 \\
$B$ & 54 & 300 & 94 & 390
\end{tabular}

\begin{tabular}{rrrrr}
55 & 240 & $\angle 27$ & 930 \\
\hline 56 & 450 & 87 & 3200 \\
57 & $<50$ & 55 & 3130 \\
58 & 90 & 55 & 3125 \\
59 & 110 & $<22$ & 3030 \\
$E \quad$ & 180 & 47 & 3465 \\
60 & 450 & $<27$ & 3510 \\
61 & 80 & 75 & 3650 \\
62 & 60 & 83 & 3450 \\
63 & 100 & 39 & 3360 \\
64 & 60 & 227 & 3135 \\
65 & $<50$ & 419 & 500 \\
67 & 170 & 82 & 605 \\
70 & 160 & 66 & 640 \\
71 & 170 & 62 & 550 \\
72 & 130 & 71 & 590 \\
73 & & &
\end{tabular}


DH -3

SC CR CO BA LA SM EU TB HF TA TH $\begin{array}{llllllllllll}2001 & -43 & 190 & 60 & 602 & 36 & 9.1 & 2.8 & 1.4 & 6.1 & 2.5 & 1.7\end{array}$

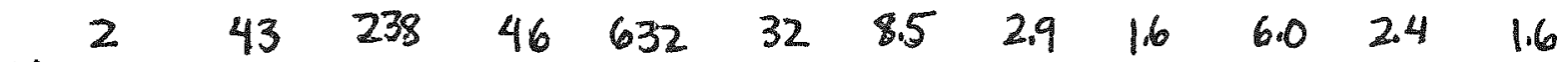

(1) $3 \quad \begin{array}{lllllllllll}45 & 185 & 49 & 626 & 39 & 9.9 & 3.3 & 1.7 & 7.5 & 26 & 1.9\end{array}$

A

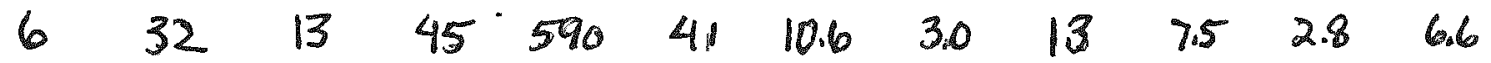
(z)

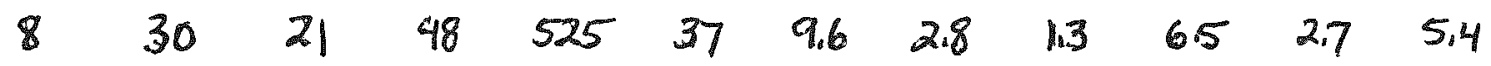
$9 \begin{array}{llllllllllll}20 & 19 & 46 & 500 & 33 & 93 & 2.7 & 1.4 & 98 & 2.6 & 5.9\end{array}$

$\begin{array}{llllllllllll}11 & 34 & 114 & 44 & 300 & 17 & 4.7 & 1.5 & 78 & 28 & 1.11 & 2.1\end{array}$

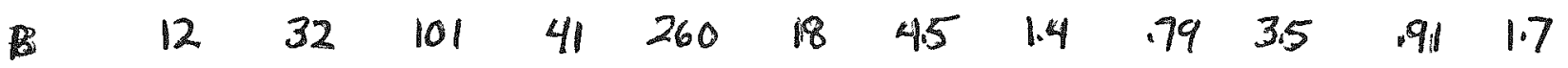

$\begin{array}{llllllllllll}13 & 32 & 111 & 42 & 250 & 17 & 49 & 1.5 & .71 & 3.3 & 1.0 & 2.3\end{array}$

$\begin{array}{llllllllllll}14 & 34 & 94 & 42 & 275 & 16 & 4.7 & 1.5 & .49 & 3.1 & 1.1 & 2.6\end{array}$

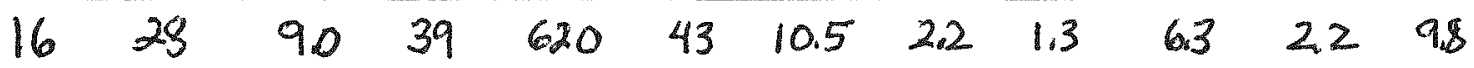

$\begin{array}{lllllllllllll}C & 17 & 31 & 8.3 & 45 & 580 & 38 & 11.4 & 2.3 & 1.0 & 5.8 & 22 & 8.6\end{array}$

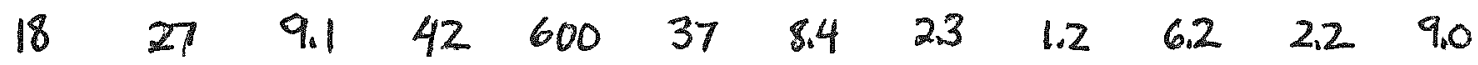

$\begin{array}{llllllllllll}19 & 28 & 50 & 42 & 670 & 38 & 8.2 & 25 & 1.1 & 50 & 2.0 & 8.2 \\ 22 & 27 & 20 & 29 & 3048 & 43 & 9.0 & 3.9 & 1.3 & 9.5 & 2.0 & 6.5\end{array}$

$\begin{array}{llllllllllll}24 & 25 & 15 & 27 & 3190 & 46 & 10.6 & 4.7 & 1.4 & 11.0 & 1.9 & 7.5\end{array}$

$\begin{array}{llllllllllll}25 & 25 & 10 & 28 & 3350 & 46 & 10.0 & 4.3 & 1.2 & 10.1 & 2.1 & 7.1\end{array}$

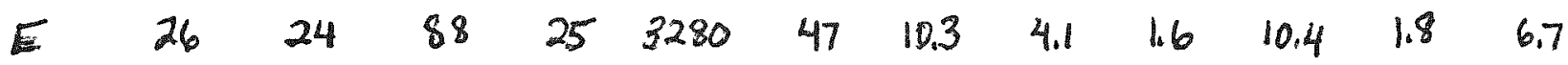

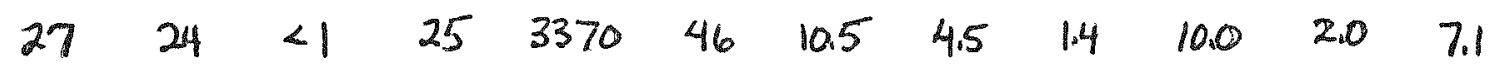

$\begin{array}{llllllllllll}28 & 26 & 41 & 27 & 3.470 & 45 & 10.0 & 4.6 & 1.7 & 11.0 & 2.4 & 7.7\end{array}$

$\begin{array}{llllllllllll}29 & 24 & 13 & 24 & 3070 & 45 & 9.0 & 4.2 & 1.9 & 13 & 2.0 & 7.5\end{array}$

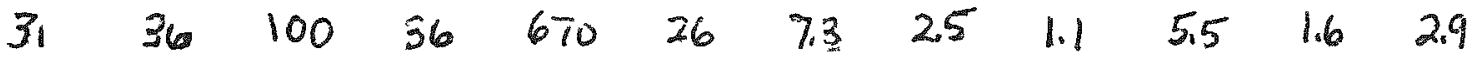

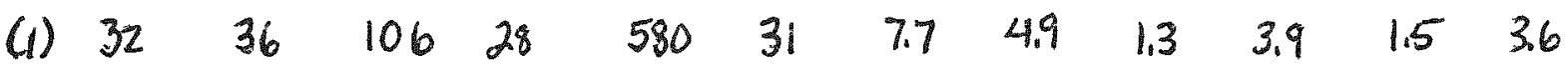

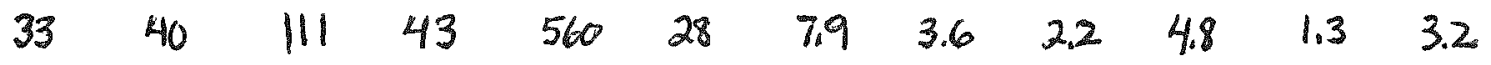

$\begin{array}{llllllllllll}34 & 36 & 100 & 38 & 540 & 26 & 78 & 2.6 & 1.5 & 48 & 1.3 & 3.8\end{array}$

$\begin{array}{llllllllllll}36 & 39 & 63 & 42 & 450 & 27 & 7.2 & 23 & 1.8 & 5.9 & 1.7 & 5.4\end{array}$

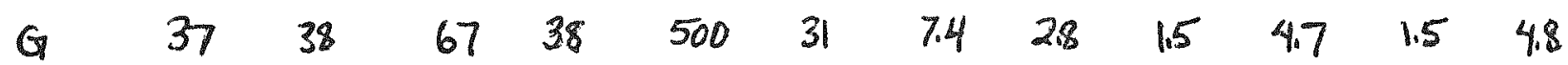

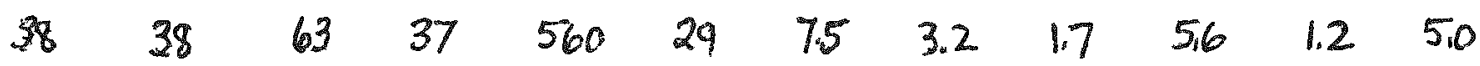

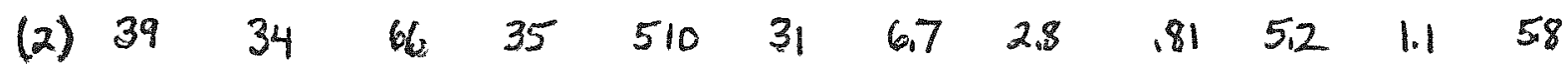

\begin{tabular}{llllllllllll}
40 & 37 & 57 & 39 & 580 & 27 & 7.0 & 2.8 & 1.6 & 5.4 & 1.2 & 35 \\
\hline 1 & 37 & 31 & 40 & 580 & 25 & 7.2 & 22 & 1.6 & 6.6 & 1.8 & 6.1
\end{tabular}

90

(3) 91

$\begin{array}{llllllllllll}43 & 33 & 18 & 35 & 715 & 27 & 7.0 & 2.0 & .94 & 5.0 & 1.4 & 4.9\end{array}$ 
$\mathrm{DH}-3$

SC CR CO BA LA SM EU TB HF TA TH $\begin{array}{llllllllllll}44 & -36 & 24 & 38 & 680 & 31 & 7.5 & 2.6 & 1.8 & 6.4 & 1.7 & 5.3\end{array}$

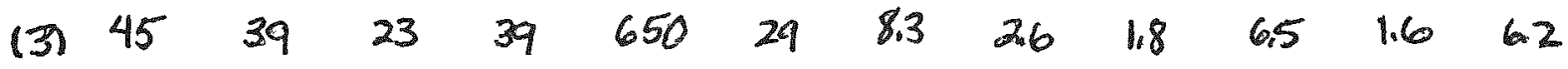

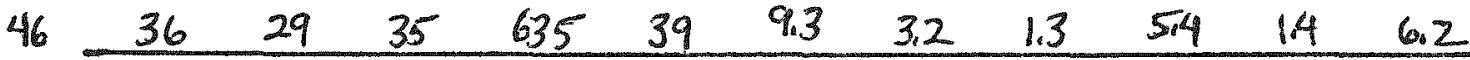

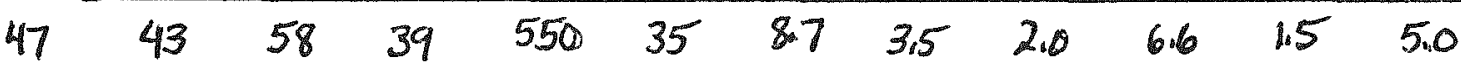
$\begin{array}{llllllllllll}48 & 39 & 56 & 40 & 550 & 31 & 7.9 & 3.2 & 1.2 & 510 & 1.4 & 6.0\end{array}$ $\begin{array}{llllllllllll}49 & 41 & 52 & 42 & 540 & 28 & 9.1 & 3.4 & 12 & 6.1 & 1.3 & 4.0\end{array}$ $\begin{array}{llllllllllll}50 & 38 & 50 & 42 & 560 & 29 & 7.3 & 2.7 & 2.0 & 53 & 1.3 & 4.4\end{array}$ (4) $51 \quad \begin{array}{lllllllllll}38 & 50 & 43 & 580 & 29 & 6.8 & 24 & 1.7 & 58 & 12 & 44\end{array}$ $\begin{array}{llllllllllll}52 & 39 & 72 & 44 & 575 & 29 & 7.4 & 23 & 1.9 & 5.4 & 13 & 4.3\end{array}$ $\begin{array}{llllllllllll}53 & 39 & 58 & 44 & 635 & 26 & 6.8 & 2.3 & 1.5 & 5.3 & 1.2 & 5.1\end{array}$ $\begin{array}{llllllllllll}54 & 38 & 60 & 39 & 460 & 20 & 5.7 & 2.1 & 1.9 & 5.4 & 1.1 & 4.2\end{array}$ $\begin{array}{llllllllllll}55 & 37 & 63 & 42 & 600 & 25 & 6.5 & 2.0 & 1.7 & 4.5 & 1.1 & 3.3\end{array}$ $\begin{array}{lllllllllllll}G & 56 & 38 & 53 & 43 & 440 & 30 & 6.9 & 23 & 1.8 & 5.4 & 1.2 & 4.2\end{array}$ \begin{tabular}{llllllllllll}
57 & 39 & 68 & 44 & 680 & 23 & 6.2 & 20 & 1.7 & 5.3 & 13 & 3.8 \\
\hline 40 & 27 & 42 & 580 & 29 & 8.3 & 2.6 & 1.9 & 4.7 & 1.3 & 4.8
\end{tabular} $\begin{array}{llllllllllll}59 & 39 & 24 & 43 & 660 & 27 & 7.3 & 2.3 & 1.9 & 4.9 & 1.3 & 4.2\end{array}$ $\begin{array}{llllllllllll}60 & 37 & 20 & 45 & 620 & 27 & 9.1 & 2.9 & 2.3 & 6.2 & 1.5 & 6.1\end{array}$

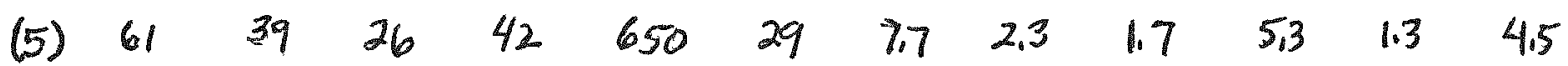
$\begin{array}{llllllllllll}62 & 40 & 32 & 44 & 700 & 27 & 7.8 & 2.4 & 1.8 & 5.6 & 1.4 & 4.5\end{array}$ $\begin{array}{llllllllllll}63 & 39 & 32 & 39 & 590 & 30 & 8.2 & 2.3 & 2.3 & 5.8 & 1.4 & 4.8\end{array}$ $\begin{array}{llllllllllll}64 & 36 & 22 & 42 & 725 & 29 & 7.7 & 2.3 & 1.8 & 5.3 & 1.1 & 4.5\end{array}$ $\begin{array}{llllllllllll}65 & 40 & 24 & 44 & 547 & 30 & 8.3 & 2.5 & 2.0 & 5.6 & 1.2 & 5.3\end{array}$ $\begin{array}{llllllllllll}66 & 39 & 27 & 44 & 588 & 29 & 7.6 & 2.2 & 1.8 & 5.1 & 1.3 & 5.5\end{array}$

$\begin{array}{llllllllllll}67 & 45 & 51 & 42 & 551 & 28 & 7.4 & 2.4 & 2.7 & 5.4 & 1.2 & 4.1\end{array}$

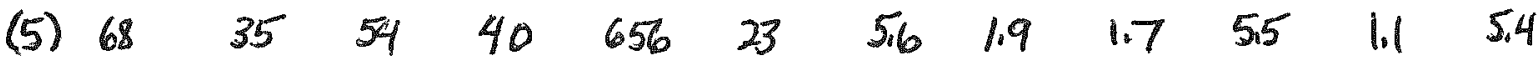
$\begin{array}{llllllllllll}69 & 37 & 59 & 23 & 553 & 46 & 11.2 & 3.0 & 2.7 & 64 & 15 & 7.1\end{array}$

$\begin{array}{llllllllllll}70 & 35 & 52 & 40 & 630 & 24 & 5.5 & 1.8 & 1.6 & 3.7 & 1.0 & 3.5\end{array}$ $\begin{array}{llllllllllll}71 & 36 & 52 & 39 & 565 & 27 & 6.2 & 1.8 & 1.7 & 6.3 & 1.2 & 5.4\end{array}$ $\begin{array}{llllllllllll}72 & 37 & 61 & 44 & 593 & 23 & 5.7 & 1.8 & 1.8 & 5.1 & .84 & 4.0\end{array}$ $\begin{array}{lllllllllllll}H & 73 & 39 & 53 & 40 & 585 & 23 & 6.2 & 1.9 & 1.4 & 5.1 & 1.1 & 5.4\end{array}$ $\begin{array}{llllllllllll}74 & 37 & 53 & 38 & 650 & 26 & 6.0 & 1.8 & 1.4 & 4.6 & 1.1 & 4.5\end{array}$

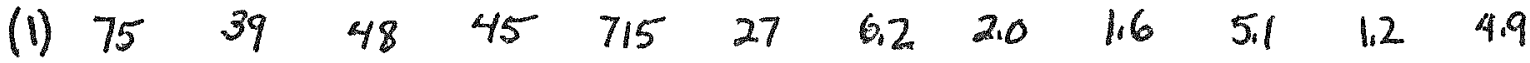
$\begin{array}{llllllllllll}76 & 35 & 42 & 39 & 765 & 28 & 6.6 & 2.0 & 1.5 & 5.0 & 1.1 & 5.0\end{array}$ $\begin{array}{llllllllllll}71 & 39 & 54 & 44 & 715 & 26 & 6.2 & 2.0 & 1.2 & 5.5 & 1.0 & 5.8\end{array}$ 
DH -3

SC CR CO BA LA SM EU TB HF TA TH

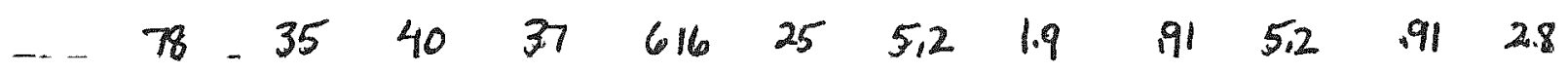
$\begin{array}{llllllllllll}79 & 40 & 48 & 42 & 591 & 22 & 6.0 & 2.0 & 1.1 & 5.4 & 1.1 & 3.5\end{array}$

(2) $\begin{array}{llllllllllll}80 & 38 & 49 & 40 & 571 & 23 & 5.5 & 2.1 & 1.0 & 5.4 & 11 & 3.1\end{array}$ $\begin{array}{llllllllllll}81 & 40 & 51 & 43 & 591 & 22 & 5.3 & 2.3 & 1.1 & 50 & 1.3 & 3.6\end{array}$

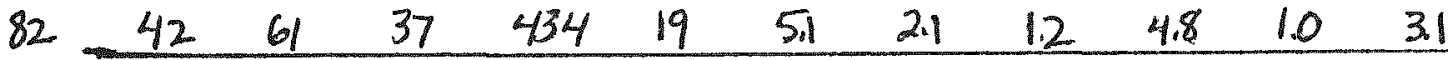

$\begin{array}{llllllllllll}83 & 40 & 49 & 45 & 475 & 19 & 5.1 & 2.0 & 1.0 & 5.7 & 1.0 & 2.9\end{array}$ $\begin{array}{llllllllllll}84 & 39 & 51 & 41 & 525 & 19 & 53 & 2.1 & 78 & 5.0 & 1.0 & 2.3\end{array}$ $\begin{array}{llllllllllll}85 & 43 & 58 & 55 & 567 & 22 & 5.4 & 2.1 & 1.2 & 5.9 & 1.0 & 29\end{array}$ $\begin{array}{llllllllllll}86 & 41 & 50 & 43 & 550 & 19 & 5.5 & 1.7 & .91 & 6.4 & 1.1 & 2.9\end{array}$

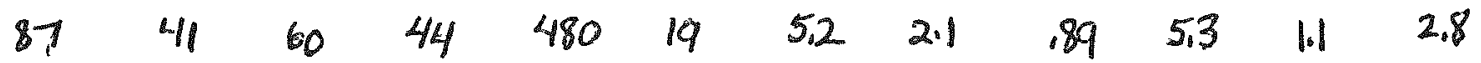
$\begin{array}{llllllllllll}88 & 30 & 41 & 32 & 570 & 15 & 4.1 & 1.5 & 177 & 3.6 & .77 & 1.9\end{array}$ $\begin{array}{lllllllllllll}H & >89 & 33 & 33 & 35 & 525 & 16 & 4.4 & 1.8 & 1.0 & 44 & 77 & 2.4\end{array}$ $\begin{array}{llllllllllll}92 & 38 & 52 & 40 & 525 & 20 & 5.0 & 2.1 & 89 & 5.0 & 89 & 2.7\end{array}$ $\begin{array}{llllllllllll}93 & 43 & 44 & 49 & 525 & 22 & 5.9 & 2.3 & 1.2 & 5.4 & 1.0 & 2.7\end{array}$

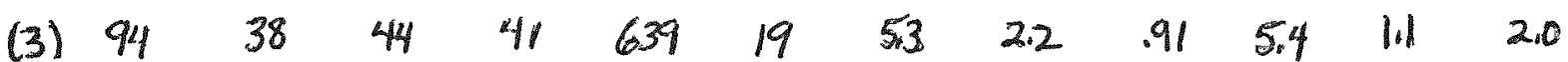
$\begin{array}{llllllllllll}95 & 36 & 40 & 40 & 716 & 20 & 5.2 & 1.9 & 1.0 & 5.0 & 1.0 & 2.7\end{array}$ $\begin{array}{llllllllllll}96 & 38 & 40 & 44 & 867 & 18 & 5.0 & 1.8 & .89 & 4.7 & 1.0 & 2.4\end{array}$ $\begin{array}{llllllllllll}97 & 42 & 47 & 46 & 472 & 17 & 510 & 19 & .93 & 5.1 & 187 & 25\end{array}$ $\begin{array}{llllllllllll}98 & 38 & 40 & 40 & 835 & 18 & 5.0 & 2.0 & 1.1 & 5.2 & .94 & 2.9\end{array}$ $\begin{array}{llllllllllll}99 & 43 & 39 & 49 & 525 & 18 & 5.1 & 2.1 & 1.0 & 6.1 & 1.0 & 2.4\end{array}$

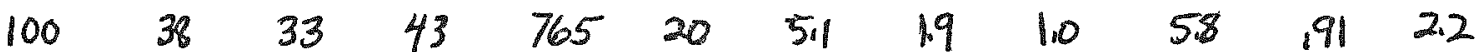
$\begin{array}{llllllllllll}101 & 42 & 33 & 49 & 570 & 21 & 5.5 & 20 & 1.0 & 64 & 1.1 & 2.7\end{array}$ $\begin{array}{llllllllllll}102 & 37 & 39 & 41 & 620 & 17 & 5.1 & 1.8 & 1.0 & 4.8 & 182 & 2.4\end{array}$ $\begin{array}{llllllllllll}103 & 42 & 28 & 41 & 570 & 23 & 65 & 2.2 & 1.0 & 6.2 & 1.1 & 3.6\end{array}$ $\begin{array}{llllllllllll}104 & 36 & 28 & 42 & 825 & 23 & 5.1 & 1.9 & 1.0 & 5.6 & .82 & 2.7\end{array}$ $\begin{array}{llllllllllll}105 & 40 & 30 & 44 & 950 & 24 & 5.7 & 2.1 & 1.1 & 6.5 & 1.1 & 3.2\end{array}$ \begin{tabular}{llllllllllll}
106 & 37 & 23 & 39 & 955 & 20 & 5.3 & 2.0 & 1.0 & 5.1 & 1.0 & 3.1 \\
\hline 108 & 38 & 22 & 36 & 980 & 29 & 7.0 & 2.7 & 1.4 & 7.9 & 1.1 & 5.6
\end{tabular}

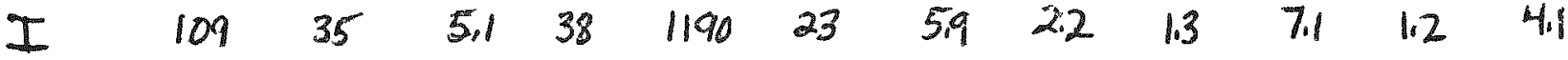
$\begin{array}{llllllllllll}110 & 38 & 13 & 41 & 760 & 28 & 6.7 & 2.4 & 1.1 & 5.6 & 1.2 & 4.2\end{array}$ 
$\mathrm{DH}-4$

SC CR CO BA LA SM EU TB HF TA TH

4001

5911

A 2

1280

$\begin{array}{llllllllllll}3 & 32 & 29 & 42 & 613 & 36 & 8.3 & 2.4 & 1.4 & 7.6 & 3.7 & 5.0\end{array}$

4

$\begin{array}{llllllllllll}5 & 33 & 121 & 40 & 406 & 18 & 3.6 & 1.4 & .70 & 23 & 1.0 & 1.9\end{array}$

6

$\begin{array}{llllllllllll}7 & 34 & 108 & 41 & 453 & 20 & 4.3 & 1.3 & 1.0 & 4.2 & 1.3 & 1.6\end{array}$

$8 \quad 467$

$\begin{array}{llllllllllll}9 & 33 & 92 & 42 & 4.67 & 18 & 4.2 & 1.6 & 1.3 & 5.1 & 1.4 & 1.6\end{array}$

$\begin{array}{lllllllllllll}B & 10 & 27 & 111 & 38 & 772 & 35 & 5.8 & 1.5 & 1.3 & 5.6 & 1.6 & 5.1\end{array}$

$\begin{array}{llllllllllll}11 & 26 & 125 & 40 & 642 & 33 & 5.5 & 1.7 & 1.1 & 5.6 & 1.6 & 2.2\end{array}$

$\begin{array}{llllllllllll}12 & 30 & 295 & 42 & 459 & 13 & 3.0 & 1.1 & 184 & 3.5 & 82 & 43\end{array}$

$\begin{array}{llllllllllll}13 & 28 & 269 & 45 & 311 & 13 & 3.2 & 1.0 & .45 & 2.8 & 1.0 & .90\end{array}$

$\begin{array}{llllllllllll}14 & 26 & 173 & 39 & 525 & 26 & 4.7 & 1.4 & 188 & 5.0 & 1.2 & 1.3\end{array}$

$\begin{array}{llllllllllll}15 & 26 & 97 & 37 & 775 & 35 & 5.9 & 1.7 & 1.2 & 7.1 & 1.7 & 2.8\end{array}$

$\begin{array}{llllllllllll}16 & 25 & 31 & 37 & 1079 & 45 & 7.2 & 1.7 & 1.1 & 7.9 & 1.7 & 3.0\end{array}$

$\begin{array}{llllllllllll}17 & 26 & 41 & 38 & 998 & 42 & 6.3 & 2.1 & 1.4 & 8.1 & 1.9 & 3.4\end{array}$

$\begin{array}{llllllllllll}18 & 34 & 92 & 38 & 1733 & 26 & 6.6 & 22 & 1.3 & 4.4 & 1.7 & 1.8\end{array}$

$\begin{array}{llllllllllll}19 & 36 & 101 & 40 & 656 & 26 & 7.11 & 25 & 1.9 & 5.5 & 1.9 & 1.8\end{array}$

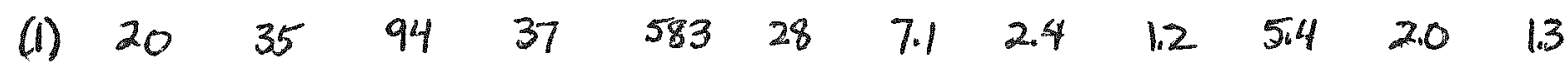

$\begin{array}{llllllllllll}21 & 35 & 103 & 40 & 525 & 27 & 6.6 & 2.1 & 95 & 4.6 & 1.1 & 28\end{array}$

$\begin{array}{lllllllllllll}F & 22 & 35 & 11 & 38 & 5.5 & 33 & 7.8 & 25 & 1.7 & 5.5 & 1.6 & 3.7\end{array}$

$\begin{array}{llllllllllll}23 & 35 & 19 & 38 & 708 & 31 & 7.7 & 2.3 & 1.1 & 5.4 & 1.2 & 3.3\end{array}$

(2) $\begin{array}{llllllllllll}24 & 36 & 16 & 38 & 6.20 & 35 & 8.3 & 2.7 & 1.6 & 5.0 & 1.6 & 3.3\end{array}$

$\begin{array}{llllllllllll}25 & 35 & 17 & 37 & 642 & 31 & 7.7 & 2.3 & 1.2 & 5.1 & 1.2 & 2.7\end{array}$

$\begin{array}{llllllllllll}26 & 35 & 20 & 37 & 727 & 33 & 7.7 & 25 & 1.4 & 3.9 & 1.6 & 3.5\end{array}$

$\begin{array}{llllllllllll}27 & 35 & 18 & 38 & 731 & 30 & 7.7 & 24 & .78 & 5.11 & 1.2 & 2.6\end{array}$

$\begin{array}{llllllllllll}28 & 34 & 48 & 36 & 607 & 33 & 7.0 & 2.2 & 1.3 & 4.0 & 1.5 & 3.5\end{array}$

$\begin{array}{llllllllllll}29 & 34 & 52 & 33 & 700 & 28 & 7.1 & 1.9 & 1.0 & 4.2 & 1.1 & 2.8\end{array}$

(1) $\begin{array}{llllllllllll}30 & 34 & 55 & 38 & 4.73 & 30 & 6.5 & 2.1 & 1.3 & 4.6 & 1.4 & 3.3\end{array}$

$\begin{array}{llllllllllll}31 & 34 & 51 & 37 & 700 & 29 & 6.8 & 1.9 & 1.1 & 4.5 & 1.0 & 2.6\end{array}$

$\begin{array}{llllllllllll}32 & 37 & 38 & 39 & 597 & 33 & 7.0 & 2.2 & 1.3 & 5.4 & 1.4 & 4.1\end{array}$

$G \quad 33$\begin{tabular}{llllllllllll}
35 & 40 & 36 & 662 & 29 & 6.6 & 2.2 & 1.5 & 3.6 & 1.3 & 3.5 \\
\hline 34 & 28 & 37 & 581 & 29 & 7.2 & 1.9 & 1.2 & 6.0 & 1.4 & 5.2
\end{tabular}

$\begin{array}{llllllllllll}35 & 33 & 28 & 38 & 710 & 29 & 6.4 & 2.2 & 1.3 & 4.1 & 1.2 & 3.7\end{array}$

(2) 36

$\begin{array}{llllllllllll}37 & 34 & 29 & 35 & 765 & 30 & 79 & 2.0 & 1.6 & 6.1 & 1.4 & 5.4\end{array}$

$\begin{array}{llllllllllll}38 & 33 & 29 & 32 & 716 & 32 & 7.5 & 2.4 & 1.1 & 5.2 & 1.3 & 4.7\end{array}$ 
DH-4

SC CR CO BA LA SM EU TB HF TA TH 39 753

$\begin{array}{llllllllllll}40 & 31 & 25 & 37 & 722 & 35 & 8.3 & 1.9 & 1.5 & 6.9 & 1.4 & 4.8\end{array}$

$\begin{array}{llllllllllll}41 & 35 & 18 & 39 & 700 & 29 & 7.1 & 26 & 1.7 & 4.8 & 1.4 & 4.4\end{array}$

$G$

\section{$42 \quad 639$}

$\begin{array}{llllllllllll}43 & 33 & 17 & 35 & 616 & 28 & 6.8 & 1.8 & 1.5 & 5.8 & 1.2 & 4.7\end{array}$ $\begin{array}{llllllllllll}44 & 36 & 20 & 36 & 677 & 28 & 6.0 & 2.4 & 1.4 & 4.1 & 1.3 & 3.4\end{array}$

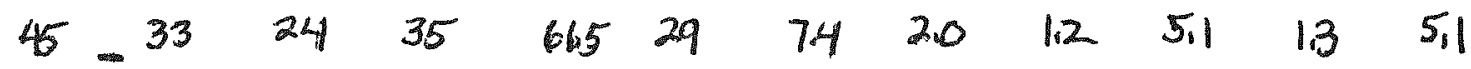
$46-610$

47

683

48

767

49

758

50

639

51

729

52

656

53

758

54

700

55

642

56

606

57

709

58

687

59

713

60

619

61

801

62

683

63

675

$\begin{array}{ll}64 & 638 \\ 65 & 600\end{array}$

$\begin{array}{llllllllllll}66 & 32 & 42 & 35 & 668 & 25 & 49 & 1.8 & 1.4 & 3.3 & 10 & 2.7\end{array}$

$\begin{array}{llllllllllll}67 & 33 & 44 & 36 & 646 & 22 & 5.0 & 1.7 & 1.1 & 4.1 & 1.7 & 3.5\end{array}$ $\begin{array}{llllllllllll}68 & 33 & 48 & 38 & 613 & 24 & 4.5 & 1.7 & 1.0 & 3.4 & 89 & 4.0\end{array}$ $\begin{array}{lllllllllllll}H & 69 & 34 & 55 & 39 & 591 & 20 & 4.6 & 1.6 & 1.0 & 3.9 & 1.0 & 29\end{array}$ $\begin{array}{llllllllllll}70 & 34 & 52 & 38 & 563 & 22 & 4.8 & 1.5 & 1.1 & 3.4 & 1.0 & 3.2\end{array}$ $\begin{array}{llllllllllll}71 & 33 & 57 & 36 & 630 & 20 & 4.6 & 1.4 & 1.0 & 3.3 & .93 & 2.7\end{array}$ 72 600

692

73

$\begin{array}{llllllllllll}74 & 35 & 53 & 37 & 656 & 23 & 5.0 & 1.6 & 91 & 3.2 & 1.0 & 3.4\end{array}$ 75 700 
$\mathrm{DH}-4$

$S C \quad C R \quad C O$ BA LA SM EL TB HF TA TH

$\begin{array}{llllllllllllll}77 & 17 & 36 & 69 & 39 & 667 & 20 & 5.2 & 1.7 & 1.4 & 4.0 & 1.1 & 1.0\end{array}$

79

80

$81 \quad 34 \quad 50 \quad 36$ 692

640

82

83

$\begin{array}{llll}84 & 33 & 41 & 36\end{array}$

85

$\begin{array}{lllllllllllll}H & 86 & 29 & 16 & 34 & 610 & 31 & 6.5 & 2.0 & 1.2 & 5.4 & 1.1 & 5.6\end{array}$ 87

$\begin{array}{llllllllllll}88 & 35 & 42 & 38 & 612 & 20 & 4.8 & 1.6 & 1.1 & 3.9 & 1.3 & 3.4\end{array}$ $89: 563$

$\begin{array}{llllllllllll}90 & 36 & 45 & 39 & 578 & 19 & 4.8 & 1.5 & 91 & 4.1 & 176 & 2.9\end{array}$ $\begin{array}{llllllllllll}91 & 35 & 45 & 38 & 591 & 21 & 5.2 & 1.7 & \text { 91 } & 4.3 & 1.2 & 4.0\end{array}$ 92 578

$\begin{array}{llllllllllll}93 & 35 & 78 & 38 & 569 & 21 & 4.9 & 1.6 & 79 & 4.3 & 1.78 & 3.3\end{array}$ $\begin{array}{llllllllllll}94 & 35 & 40 & 39 & 691 & 20 & 5.1 & 1.7 & 91 & 3.7 & 1.3 & 2.8\end{array}$ $\begin{array}{llllllllllll}95 & 37 & 24 & 40 & 760 & 22 & 5.2 & 1.7 & 1.2 & 5.0 & 1.1 & 3.2\end{array}$ $96 \quad 735$

$\begin{array}{llllllllllll}97 & 33 & 15 & 39 & 758 & 25 & 5.6 & 1.9 & 778 & 5.3 & 1.4 & 5.6\end{array}$ $\begin{array}{llllllllllll}98 & 33 & 17 & 39 & 6.22 & 24 & 5.4 & 1.8 & 1.0 & 4.7 & 1.0 & 3.7\end{array}$ $\begin{array}{llllllllllll}99 & 33 & 15 & 37 & 678 & 28 & 5.7 & 1.9 & .92 & 5.5 & 1.3 & 4.3\end{array}$ 100 623

$101 \quad 803$

$\begin{array}{llllllllllll}102 & 32 & 13 & 35 & 716 & 28 & 6.0 & 1.7 & 89 & 5.3 & 1.1 & 4.4\end{array}$ $\begin{array}{llllllllllll}103 & 32 & 22 & 36 & 788 & 28 & 6.0 & 1.8 & 1.1 & 3.9 & 1.0 & 3.9\end{array}$ $\begin{array}{llllllllllll}104 & 32 & 11 & 35 & 830 & 27 & 6.1 & 1.9 & 187 & 5.0 & 1.3 & 4.9\end{array}$ $105 \quad 810$

$106 \quad 720$

I $\quad \begin{array}{llllllllllll}107 & 30 & 26 & 35 & 740 & 27 & 5.9 & 1.6 & 1.0 & 4.3 & 1.1 & 5.0\end{array}$ 108

$\begin{array}{llllllllllll}109 & 30 & 7.3 & 34 & 670 & 30 & 5.9 & 1.9 & .76 & 5.0 & 1.3 & 5.1\end{array}$ $\begin{array}{llllllllllll}110 & 29 & 28 & 35 & 680 & 28 & 6.0 & 1.8 & 1.85 & 5.3 & 1.1 & 4.8\end{array}$ $\begin{array}{llllllllllll}111 & 29 & 12 & 33 & 710 & 30 & 5.8 & 1.8 & 48 & 5.3 & 1.3 & 4.4\end{array}$ $\begin{array}{llllllllllll}112 & 29 & 8.6 & 35 & 770 & 28 & 5.9 & 1.7 & .92 & 4.7 & 1.2 & 4.9\end{array}$ 113 690 114 
DH -4

$S C$ CR CO BA LA SM EU TB HF TA TH

115

116

117

750

118

$\begin{array}{llllllllll}13 & 34 & 680 & 29 & 59 & 1.8 & .91 & 5.5 & 1.2 & 5.4\end{array}$

850

$\begin{array}{llllllllllll}119 & 31 & 21 & 36 & 750 & 29 & 6.1 & 1.8 & 1.0 & 5.7 & 1.2 & 4.7\end{array}$

$120 \quad 780$

$\begin{array}{llllllllllll}121 & 29 & 24 & 34 & 780 & 30 & 6.0 & 1.9 & 1.0 & 5.4 & 1.2 & 5.8\end{array}$

$\begin{array}{llllllllllll}122 & 30 & 42 & 36 & 730 & 30 & 60 & 1.8 & 1.1 & 5.5 & 1.2 & 5,0\end{array}$

(1) 123

$\begin{array}{llllllllllll}124 & 31 & 16 & 35 & 800 & 28 & 5.9 & 1.9 & 1.0 & 5.1 & 1.3 & 5.1\end{array}$

I $\quad 125$

$\begin{array}{llllllllllll}126 & 29 & 78 & 34 & 800 & 29 & 6.2 & 1.7 & 175 & 4.2 & 1.0 & 5.2\end{array}$

127

$\begin{array}{llllllllllll}128 & 30 & 16 & 34 & 810 & 33 & 6.3 & 1.9 & 1.0 & 5.1 & 13 & 7.0\end{array}$

129

130

$13 !$

$\begin{array}{llllllllllll}132 & 29 & 9 & 34 & 610 & 28 & 5.7 & 1.8 & 10 & 5.2 & 1.2 & 3.8\end{array}$

$\begin{array}{llllllllllll}133 & 29 & 11 & 34 & 620 & 29 & 5.8 & 1.9 & 1.0 & 5.2 & 1.2 & 7.2\end{array}$

(2) $\begin{array}{llllllllllll}134 & 29 & 18 & 34 & 600 & 28 & 5.9 & 1.8 & 1.4 & 5.6 & 1.3 & 4.9\end{array}$

$\begin{array}{llllllllllll}135 & 34 & 16 & 40 & 600 & 29 & 6.6 & 2.2 & 1.2 & 6.2 & 1.5 & 5.9\end{array}$

$\begin{array}{llllllllllll}136 & 33 & 42 & 37 & 600 & 27 & 6.2 & 1.9 & 1.7 & 4.9 & 1.3 & 2.9\end{array}$

$\begin{array}{llllllllllll}137 & 32 & 37 & 37 & 600 & 28 & 6.1 & 1.9 & 1.0 & 4.9 & 1.4 & 4.6\end{array}$

$\begin{array}{llllllllllll}138 & 32 & 19 & 36 & 640 & 25 & 6.1 & 2.0 & 1.3 & 50 & 1.3 & 4.4\end{array}$

$139 \quad 630$

$\begin{array}{llllllllllll}140 & 31 & 36 & 37 & 570 & 25 & 5.4 & 1.7 & .72 & 3.7 & 1.1 & 5.2\end{array}$

$\begin{array}{llllllllllll}141 & 31 & 27 & 35 & 680 & 21 & 5.1 & 1.5 & 1.0 & 3.9 & 1.1 & 3.9\end{array}$

$\begin{array}{llllllllllll}142 & 31 & 30 & 36 & 640 & 22 & 4.9 & 1.5 & 1.6 & 3.3 & 1.3 & 2.2\end{array}$

$\begin{array}{llllllllllll}143 & 30 & 24 & 34 & 640 & 26 & 5.3 & 1.7 & 1.5 & 4.5 & 1.5 & 2.7\end{array}$

1444660

$\begin{array}{lllllllllllll}J & 145 & 30 & 9 & 35 & 630 & 26 & 5.5 & 1.5 & 2.5 & 4.5 & 1.4 & 1.3\end{array}$

$\begin{array}{llllllllllll}146 & 30 & 23 & 34 & 580 & 27 & 5.4 & 1.6 & 1.5 & 4.5 & 1.5 & 3.0\end{array}$

$\begin{array}{llllllllllll}147 & 31 & 30 & 35 & 500 & 26 & 5.7 & 1.5 & 23 & 5.3 & 1.5 & 2.3\end{array}$

$\begin{array}{llllllllllll}148 & 31 & 13 & 36 & 860 & 26 & 5.6 & 1.8 & 1.4 & 4.7 & 1.8 & 3.3\end{array}$

$\begin{array}{llllllllllll}149 & 30 & 17 & 33 & 700 & 26 & 5.4 & 1.5 & 1.2 & 5.6 & 1.6 & 2.9\end{array}$

$150 \quad 690$

$\begin{array}{llllllllllll}151 & 29 & 13 & 34 & 720 & 26 & 5.2 & 1.5 & 193 & 4.1 & 1.5 & 3.1\end{array}$

$\begin{array}{llllllllllll}152 & 30 & 15 & 33 & 700 & 26 & 5.4 & 1.7 & 1.3 & 3.9 & 1.8 & 4.0\end{array}$ 
$\mathrm{DH}-4$

SC CR CO BA LA SM EU TB. HF TA TH

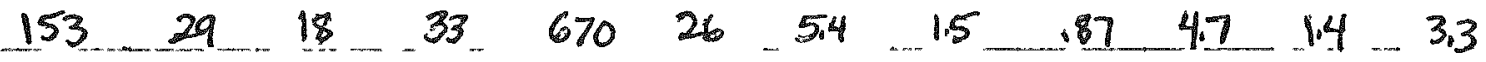
$\begin{array}{llllllllllll}154 & 29 & 76 & 32 & 740 & 27 & 5.6 & 1.6 & 77 & 4.6 & 1.8 & 4.4\end{array}$ $\begin{array}{llllllllllll}155 & 28 & 8.6 & 33 & 620 & 25 & 5.2 & 1.3 & .93 & 4.6 & 1.4 & 3.1\end{array}$ $156 \quad 780$

$\begin{array}{lllllllllllll}\text { J } & 157 & 29 & 11 & 33 & 790 & 25 & 5.3 & 1.4 & 169 & 4.3 & 1.4 & 4.7\end{array}$ $\begin{array}{llllllllllll}158 & 28 & 12 & 33 & 720 & 25 & 5.1 & 1.4 & 1.2 & 4.5 & 1.3 & 3.3\end{array}$ $\begin{array}{llllllllllll}159 & 30 & 13 & 34 & 740 & 28 & 5,5 & 1.6 & 1.3 & 5,0 & 2,1 & 4,5\end{array}$

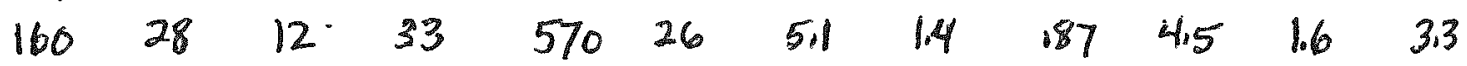
$\begin{array}{llllllllllll}161 & 30 & 44 & 34 & 590 & 28 & 55 & 1.7 & 181 & 3.9 & 2.0 & 4.7\end{array}$ $\begin{array}{llllllllllll}162 & 29 & 19 & 34 & 640 & 26 & 5.3 & 1.9 & 1.4 & 5.2 & 1.8 & 3.1\end{array}$ $\begin{array}{llllllllllll}163 & 30 & 8.3 & 34 & 610 & 25 & 5.8 & 2.0 & 1.0 & 6.5 & 1.9 & 3.2 \ldots\end{array}$ $164 \quad 850$

$\begin{array}{llllllllllll}165 & 32 & 10 & 36 & 740 & 28 & 6.2 & 2.1 & 1.4 & 6.3 & 1.7 & 3.1\end{array}$ $\begin{array}{llllllllllll}166 & 31 & 16 & 35 & 900 & 27 & 6.4 & 2.1 & 1.5 & 6.4 & 1.8 & 3.0\end{array}$ $167 \quad 650$

$\begin{array}{lllllllllllll}K & 168 & 31 & 23 & 36 & 640 & 26 & 5.8 & 2.1 & 1.1 & 6.3 & 2.2 & 2.5\end{array}$ $\begin{array}{llllllllllll}169 & 32 & 10 & 36 & 450 & 26 & 6.7 & 2.3 & 1.6 & 6.1 & 2.1 & 2.7\end{array}$ $\begin{array}{llllllllllll}170 & 33 & 17 & 38 & 650 & 27 & 5.9 & 1.9 & 1.2 & 5.8 & 2.1 & 3.0\end{array}$ $\begin{array}{llllllllllll}171 & 31 & 21 & 35 & 620 & 25 & 6.2 & 2.2 & 1.3 & 7.5 & 1.7 & 2.6\end{array}$ 172 560

$\begin{array}{llllllllllll}173 & 32 & 19 & 37 & 570 & 26 & 5.8 & 2.0 & 1.0 & 5.8 & 2.2 & 2.9\end{array}$ 
DH -5

SC CR CO BA LA SM EU TB HF TA TH 5001

$\begin{array}{llllllllllll}2 & 28 & 23 & 34 & 687 & 45 & 9.4 & 2.6 & 1.5 & 5.3 & 2,2 & 5.9\end{array}$ $\begin{array}{lllllllllllll}A & 3 & 30 & 29 & 4.5 & 551 & 38 & 8.2 & 2.6 & 1.7 & 6.9 & 2.2 & 5.2\end{array}$ $\begin{array}{llllllllllll}4 & 30 & 22 & 45 & 616 & 40 & 8.7 & 2.3 & 1.4 & 6.7 & 2.2 & 5.6\end{array}$ $\begin{array}{llllllllllll}5 & 29 & 26 & 47 & 1176 & 38 & 8,3 & 2.4 & 1.4 & 7.4 & 2.1 & 46\end{array}$

$\begin{array}{llllllllllll}7 & 32 & 96 & 40 & 389 & 19 & 4.2 & 1.4 & 1.1 & 3.2 & 1.0 & 2.7\end{array}$ 8

$\begin{array}{llllllllllll}9 & 32 & 106 & 41 & 385 & 18 & 4.0 & 1.4 & 10 & 3.1 & 1.0 & 2.3\end{array}$ 10

$\begin{array}{lllllllllllll}1 & 11 & 33 & 104 & 43 & 4.4 & 21 & 4.3 & 1.4 & .89 & 3.3 & 1.0 & 3.0\end{array}$ $12 \quad 350$

$\begin{array}{llllllllllll}13 & 26 & 217 & 43 & 411 & 16 & 3.6 & 1.4 & .78 & 3.2 & .69 & 1.7\end{array}$ $\begin{array}{llllllllllll}14 & 27 & 202 & 42 & 490 & 25 & 4.5 & 1.3 & 1.0 & 3.2 & .77 & 2.6\end{array}$ $\begin{array}{llllllllllll}15 & 29 & 198 & 42 & 525 & 26 & 4.9 & 14 & 1.1 & 4.4 & .94 & 3.6\end{array}$ $16 \quad 4 / 85$

\begin{tabular}{llllllllllll}
17 & 27 & 167 & 41 & 551 & 27 & 5.0 & 1.3 & 1.2 & 33 & .93 & 3.0 \\
\hline 18 & 34 & 95 & 37 & 600 & 26 & 6.9 & 2.3 & 1.0 & 4.7 & 1.2 & 2.9
\end{tabular}

(1) $19 \begin{array}{lllllllllll}35 & 105 & 38 & 554 & 24 & 6.3 & 2.1 & 1.5 & 32 & 1.2 & 2.1\end{array}$

$\begin{array}{llllllllllll}20 & 37 & 21 & 38 & 636 & 30 & 7.6 & 2.4 & 11.5 & 55 & 1.6 & 4.3\end{array}$

$\begin{array}{llllllllllll}21 & 36 & 15 & 37 & 689 & 29 & 7.8 & 2.3 & 1.7 & 4.0 & 1.5 & 3.4\end{array}$

$\begin{array}{llllllllllll}22 & 36 & 20 & 39 & 642 & 33 & 8.4 & 2.5 & 1.2 & 5.8 & 1.7 & 3.4\end{array}$ F 232788

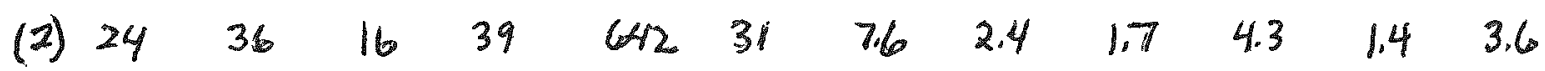
$25 \quad 700$

$\begin{array}{llllllllllll}26 & 37 & 21 & 38 & 700 & 32 & 8.5 & 2.5 & 1.4 & 6.1 & 1.6 & 4.2\end{array}$ $\begin{array}{llllllllllll}27 & 29 & 55 & 33 & .710 & 23 & 5.3 & 1.7 & 1.4 & 3.0 & 1.0 & 2.5\end{array}$ $28 \quad 656$

$\begin{array}{llllllllllll}29 & 40 & 21 & 411 & 750 & 33 & 8.3 & 2.5 & 1.6 & 6.1 & 1.7 & 4.6\end{array}$ $\begin{array}{llllllllllll}30 & 34 & 61 & 37 & 755 & 27 & 6.4 & 2.2 & 1.2 & 4.6 & 1.2 & 2.7\end{array}$ $\begin{array}{llllllllllll}31 & 35 & 44 & 37 & 744 & 27 & 6.3 & 2.1 & 1.1 & 4.2 & 1.3 & 1.8\end{array}$ G $\quad 32$

$\begin{array}{llllllllllll}33 & 31 & 26 & 34 & 805 & 26 & 6.3 & 2.2 & 1.2 & 4.6 & 1.0 & 3.3\end{array}$ 34 670 35 683 $\begin{array}{llll}36 & 625\end{array}$

$\begin{array}{llllllllllll}37 & 40 & 36 & 43 & 687 & 31 & 8.0 & 2.5 & 1.0 & 5.3 & 1.6 & 4.5\end{array}$ 38 680 
$\mathrm{DH}-5$

SC CR CO BA LA SM EU TB HF TA TH 39 718

40

735

41

42

43

44

45

46

47

G 48

$49 \quad 36-56-39 \quad 805$

\section{$50 \quad 540$}

$\begin{array}{lllll}51 & 34 & 86 & 39 & 500\end{array}$

52

53

54

55

56

57

58

59

$\begin{array}{lllll}60 & 35 & 29 & 40 & 480\end{array}$

61

$\begin{array}{lllll}62 & 35 & 26 & 37 & 520\end{array}$

$63 \quad 640$

$\begin{array}{lllll}64 & 34 & 72 & 37 & 520\end{array}$

65

66

\section{6}

6

69

H 7

\section{2}

73

$\begin{array}{llll}74 & 33 & 41 & 36\end{array}$

$\begin{array}{llll}75 & 35 & 50 & 38\end{array}$

76

788 675

710 770 550 550

510 530

520

460 500 530 540 500 $\begin{array}{llllllll}81.5 & 31 & 7.1 & 1.9 & 1.6 & 4.9 & 2.1 & 3.1\end{array}$

$\begin{array}{lllllll}28 & 6.5 & 1.9 & 1.6 & 60 & 20 & 28\end{array}$ $\begin{array}{llllll}6.3 & 2.1 & 1.4 & 5.2 & 1.9 & 3.1\end{array}$

$\begin{array}{llllllll}805 & 28 & 6.4 & 1.9 & 1.5 & 5.4 & 2.1 & 2.7\end{array}$ $\begin{array}{llllllll}500 & 25 & 6.1 & 2.1 & 1.4 & 7.7 & 2.0 & 3.0\end{array}$ $\begin{array}{llllllll}470 & 29 & 6.9 & 2.0 & 1.4 & 4.4 & 1.9 & 3.7\end{array}$

$\begin{array}{llllllll}710 & 25 & 6.2 & 2.1 & 1.7 & 6.1 & 2.2 & 2.2\end{array}$

$\begin{array}{llllllll}480 & 27 & 6.4 & 2.0 & 1.5 & 3.5 & 1.6 & 3.0\end{array}$

$\begin{array}{llllllll}520 & 27 & 6.5 & 1.9 & 1.4 & 5.8 & 2.0 & 3.4\end{array}$ $\begin{array}{lllllllll}520 & 22 & 4.5 & 1.3 & 1.2 & 3.6 & 1.0 & 17\end{array}$ $\begin{array}{llllllll}650 & 23 & 5.0 & 1.6 & 1.2 & 5.0 & 1.7 & 2.3\end{array}$ $\begin{array}{llllllll}520 & 22 & 50 & 1.4 & .75 & 3.5 & 1.3 & 2.8\end{array}$ $\begin{array}{llllllll}510 & 20 & 4.6 & 1.5 & 188 & 4.1 & 1.2 & 2.6\end{array}$ $\begin{array}{llllllll}480 & 25 & 5.2 & 1.6 & 1.3 & 40 & 1.6 & 2.9\end{array}$

$\begin{array}{llllllll}560 & 22 & 47 & 14 & .82 & 6.2 & 1.5 & 2.9\end{array}$

$\begin{array}{llllllll}500 & 23 & 5.1 & 1.5 & 1.2 & 4.6 & 1.2 & 2.1\end{array}$

$\begin{array}{lllllll}19 & 4.4 & 1.5 & 1.2 & 5.4 & 1.4 & 1.7\end{array}$ $\begin{array}{lllllll}22 & 4.8 & 1.5 & 1.3 & 3.5 & 1.3 & 3.6\end{array}$ $\begin{array}{lllllll}20 & 4.6 & 1.5 & 1.5 & 4.4 & 1.2 & 1.9\end{array}$ 
TABLE I

MAJOR ELEMEIT AVERAGES AIDD RATGES OF UNITS A-L

Averages and Ranpes of Units

Units Oxides

Core Holes

$\mathrm{DH}-1$

DH -2

DH -3

DH -4

$\mathrm{DH}-5$

(2) $\%$

(5)

(a)

(3)

(5)

$52.4 \quad 51.3-53.0 \quad 52.0 \quad 50.5-53.2$

$3.2 \quad 3.1-3.4$

$12.8 \quad 11.8-13.6$

$11.8 \quad 10.7-13.2$

$14.4 \quad 13.1-15.5$

$16.214 .8-17.3$

$.2 \quad .2-.2$

$3.9 \quad 3.7-4.3$

$0.0 \quad 8.2=9.9$

CAO $8.28 .1-8.2$

IIATO $2.5 \quad 2.4-2.6$

$120 \quad 1.4 \quad 1.3-2.4$

$2.2 \quad 2.1-2.3$

$5.2 \quad 4.4-6.5$

$8.87 .9-10.6$

$2.4 \quad 2.0-2.8$

$51.6 \quad 51.2-51.9$ $3.2 \quad 3.2 \mathrm{~m} 3.3$

$12.7 \quad 12.7-12.8$

$15.6 \quad 15.1-15.8$

$.2 .2 \% .2$

$4.6 \quad 4.5-4.8$

$8.7 \quad 8.6 .9 .0$

$1.1 \quad 1.0-1.4$

$1.0 \quad .6-2.2$

$\begin{array}{rr}2.4 & 2.3-2.5 \\ .9 & .8\end{array}$

(4)

(13)

(4)

$\begin{array}{rr}52.5 & 51.8-53.5 \\ 1.6 & 1.5-1.8\end{array}$

$13.9 \quad 12.9-14.7$

$\begin{array}{rr}11.7 & 10.6-12.7 \\ .2 & .2-.2\end{array}$

7.15 .900 .0

$10.3 \quad 10.1-10.4$

$\begin{array}{rr}2.3 & 2.2-2.4 \\ .5 & .3-.7\end{array}$

$55.0 \quad 54.2-55.5$

$1.7 \quad 1.6-1.7$

$13.4 \quad 13.3-13.6$

10.9 10.6-11.1

$7.2 \quad 7.1-7.3$

$5.5-8.7$

MGO

CAO

$10.0 \quad 0.4-11.0$

reO

2.3
.5
$.3-8-2.5$

$9.2 \quad 8.7-10.1$

$2.2 \quad 2.1-2.3$

\section{(14)}

$\begin{array}{rr}52.3 & 50.2-53.9 \\ 1.7 & 1.3-1.9\end{array}$

$14.4 \quad 13.7-16.0$

$11.3 \quad 10.0-12.3$

$.2 .2-.2$

$7.2 \quad 5.2-9.4$

$9.9 \quad 8.2-10.6$

$\begin{array}{rr}2.4 & 2.2-2.8 \\ .6 & .1-2.0\end{array}$

(4)

$55.7 \quad 55.0-57.1$

$2.6 \quad 2.4-2.7$

$12.912 .3-13.3$

$14.1 \quad 13.6-14.4$

ALEn $13.3 \quad 12.2-13.7$

FEO $\quad 13.7 \quad 12.1-14.7$

C ino

$\because \mathrm{GOO}$

CAO

TAaC $\quad 2.8 \quad 2.5-2.9$

$4.2=.2-.7-4.2$

$\begin{array}{ll}4.1 & 2.7-4.2 \\ 7.7 & 7.2-8.8\end{array}$

$\begin{array}{ll}3.8 & 3.6-4.1 \\ 7.0 & 6.0-7.6 \\ 2.5 & 2.0-2.7 \\ 1.6 & 1.3-1.7\end{array}$

(6)

SIOR $\quad 52.1 \quad 51.1-52.6$

TIO2 $1.6 \quad 1.4-1.8$

AL203 $\quad 14.0 \quad 14.7-15.2$

FEO $\quad 11.3 \quad 10.6-12.0$

D MITO

MGO

$\mathrm{CAO}$

$\mathrm{NAPO}$

$\mathrm{KeO}$

$\begin{array}{rr}.2 & .1-0.2 \\ 7.4 & 6.2-8.7 \\ 0.4 & 0.0-0.8\end{array}$

$2.4 \quad 2.3-2.5$

(c)

SIO2 $\quad 55.0 \quad 53.0-56.8$

TIO2

AI203

FEO

E. 9110

$2.0 \quad 2.3-3.5$

$13.8 \quad 13.1-16.0$

$13.5 \quad 11.7-15.8$

$.2-.3$

IGO $2.92 .0-3.5$

CAO $\quad 6.3 \quad 5.3-7.0$

$\begin{array}{lll}\text { TIA2C } & 3.1 & 2.8-3.4 \\ \text { TIOC } & 2.3 & 1.6-2.8\end{array}$

(10)

$55.4 \quad 52.6-57.3$

$2.8 \quad 2.4-3.6$

$12.5 \quad 11.1-13.4$

$14.6 \quad 12.6-16.4$

.2 .2-.2

$2.7 \quad 2.4-2.9$

$6.0 \quad 5.9-7.8$

$3.0 \quad 2.6-3.2$

$2.8 \quad 2.2-3.2$
(7)

$57.2 \quad 56.3-57.9$

$2.4 \quad 2.2-2.8$

$13.1 \quad 12.6-13.8$

$12.4 \quad 11.8-13.2$

$3.2 \quad 2.6-4.4$

$5.9 \quad 5.6-6.1$

$3.2 \quad 2.9-3.4$

$2.5 \quad 2.2-2.7$

* Number in parenthesis is the number of averaped samples in the unit 
TABLE I (Continued)

CORE HOLES

$\mathrm{DH}-1$

(18)

\begin{tabular}{|c|c|c|c|c|}
\hline SIO2 & 51.9 & $50.3-54.1$ & 50.8 & $49.1-52.6$ \\
\hline TIO2 & & & & \\
\hline $\mathrm{ALCO} 3$ & 13.4 & $12 \cdot 2-14 \cdot 3$ & 11 & \\
\hline FEO & 13.8 & 126 & 16. & \\
\hline MNO & .2 & $.2-.3$ & .2 & $.2-$ \\
\hline MCO & 5.3 & $.0-6.3$ & 4.6 & $3-4$ \\
\hline CAO & 8.8 & $7.5-9.4$ & 9.3 & - \\
\hline NAEO & 2.8 & & 2.5 & $2.3-2$ \\
\hline Kan & 3 & & & \\
\hline
\end{tabular}

$\mathrm{DH}-2$
STOR

TIO2

AL2O?

FEO

G MNO

$\therefore$ SGO

$\mathrm{CAO}$

NA2O

$\mathrm{KeO}$

SI02

TTO2

ALO?

II $\mathrm{FEO}$

MNO
MCO

$\mathrm{CAO}$

NA2O

$\mathrm{KeO}$

SIO2

TIO?

Atec?

FEO

I MNO

PSOO

$\mathrm{CAO}$

MAEO

KoO

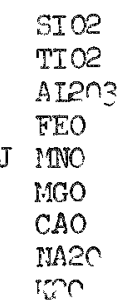

DH -3

(41)

$\begin{array}{rr}54.3 & 52.2-57.1 \\ 2.5 & 1.7 .7 .3 .0\end{array}$

$12.7 \quad 12.0-16.3$

$14.2 \quad 10.8-15.7$

$4.4 \quad 3.7=5.2$

$7.8 \quad 7.0-8.8$

$2.7 \quad 1.9-3.0$

$1.1 .8-1.4$

(36)

$55.3 \quad 51.8-58.0$

$1.7 \quad 1.5-1.9$

$13.212 .1-14.7$

$12.8 \quad 11.5-14.3$

$5.3 \quad 4.5-6.7$

$8.07 .2-9.1$

$2.82 .4-3.2$

$.9 .6-1.3$

(13)

$56.7 \quad 55.1-58.9$

$2.1 \quad 1.9-2.2$

$13.0 \quad 12.0 \times 13.7$

$12.911 .8 \mathrm{~m} 14.1$

$3.7 \quad 3.4=3.9$

$6.8 \quad 6.3=7.0$

3.2

$2.8-3.5$

$1.3-2.5$

(5)

$54.2 \quad 52.5-55.6$

$1.9 \quad 1.7-2.0$

$13.3 \quad 12.7-14.5$

$12.7 \quad 12.2-13.4$

$5.4 \quad 5.0-5.8$

$\begin{array}{ll}5.4 & 5.0=5.8 \\ 8.3 & 7.9=8.8\end{array}$

$3.1 \quad 2.9-3.2$

$1.0 \quad .9-1.1$
DH -4

DiH -5

(10)

$50.4 \quad 49.8-51.8$

$3.0 \quad 2.8-3.1$

$12.6 \quad 12.3-13.5$

$15.8 \quad 14.4-18.5$

$.3 .2-.4$

$5.4 \quad 4.7-6.1$

$8.5 \quad 7.9=9.1$

2.6

1.0

$2.5-2.8$
$.8-1.2$

(36)

$52.7 \quad 51.3-54.5$

2.7

13.1

$2.5-3.0$

$12.6-13.5$

$13 \cdot 7-16.2$

$.2 .2-.3$

4.7

7.8

2.9

$3.9=5.3$

$7.0-10.0$

$2.5-3.2$

$.5-1.8$

(35)

$54.9 \quad 53.0-56.7$

1.9

13.8

12.1

$1.6=2.2$

$12.9-14.5$

$11.3-13 \cdot 3$

$.2-.3$

$4.2-5.9$

$7 \cdot 3-9 \cdot 9$

$2.9-3.5$

$.3-1.3$

(38)

$56.6 \quad 55.1-58.1$

2.0

23.5

12.3

.2

$3 \cdot 5$

7.1

3.4

1.5

$1.7=2.3$

$12,5-15.0$

$11.2-14.8$

$.2=.2$

$3.1-4.2$

$6.7-7.6$

$2.7-3.8$

$1.0-2.7$

(24)

$57.4 \quad 55.6-59.0$

$1.81 .7-2.0$

$13.312 .5-14.1$

11.6

$10.8-12.7$

$.2-.2$

$3.6 \quad 3.2-4.3$

$7.3 \quad 6.4-8.6$

$3.4 \quad 3.2-3.7$

$1.3 \quad .9-1.9$
(10)

$52.0 \quad 50.9-54.6$

$3.0 \quad 2.8-3.5$

$13.0 \quad 12.1-13.9$

$15.0 \quad 14.2-15.6$

$5.0 \quad 4.1=6.1$

$8.17 .8-8.6$

$2.8 \quad 2.6-3.0$

$2.1 .9=1.2$

(36)

$53.0 \quad 52.0-55.0$

$2.8 \quad 2.4-3.2$

$13.0 \quad 12.2-14.5$

$14.4 \quad 12.0-15.5$

$.2 \quad .2-.3$

$4.64 .1-5.2$

$8.07 .2-8.6$

$2.8 \quad 2.6 .3 .1$

$1.2 .7-1.6$

(18)

$54.4 \quad 51.8-55.5$

$1.7 \quad 1.5 \mathrm{~m} 1.9$

$13.9 \quad 13.4-14.4$

$11.5 \quad 10.9-11.9$

$.2 .2=.2$

$5.3 \quad 4.8-6.0$

$8.8 \quad 8.3-9.2$

$3.0 \quad 2.8-3.3$

$1.3 .6-4.1$

(22)

$55.9 \quad 53.1-57.5$

$2.0 \quad 1.8-2.2$

$13.6 \quad 23 \cdot 3-14.4$

$12.111 .3-12.8$

$4.0 \quad 3.1-5.1$

$7.3 \quad 6.9-8.7$

$3.3 \quad 3.0-3.5$

$1.6 \quad 1.1-2.9$

(4)

$56.1 \quad 54.9-56.9$

$1.9 \quad 1.9-1.9$

$13.7 \quad 13.6-13.8$

$11.511 .4-11.6$

$4.1 \quad 3.9-4.3$

$7.3 \quad 7.0-7.9$

$3.4 \quad 3.3-3.4$

$2.1 \quad 1.6-2.6$ 
TABLE I (Continued)

CORE HOLES

DH -3

$\mathrm{DH}-4$

$\mathrm{DH}=5$

SIO2

TIO2

ALPO?

FEO

IF MPO

$\mathrm{MCO}$

CAO

NACO

K2O

SIOE

TIOE

ALPC3

FEO

L INNO

MCO

CAO

NA2O

$\mathrm{reO}$
(4)

$52.3 \quad 50.8-53.2 \quad 55.4 .53 .7-56$

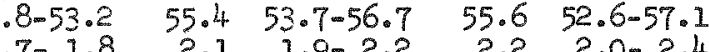
$14.6 \quad 14.2-15.1 \quad 13.5 \quad 13.0-14.1 \quad 13.5 \quad 12.6-14.3$ $11.8 \quad 11.5-12.3 \quad 12.4 \quad 12.1-13.2 \quad 12.6 \quad 11.8-13.3$ $6.3 \quad 6.0-6.6 \quad 3.8 \quad 3.6-3.9$ $9.4 \quad 9.0-9.8 \quad 8.0 \quad 7.4-10.0$ $3.3 \quad 3.2-3.3 \quad 3.5 \quad 3.3=3.6$ .6 .4 .9

1.2

8 .

(1)
$3.83 .4-4.4$

$7.16 .6-8.0$

$3.2 \quad 2.9-3.5$

$1.9 \quad .9-3.5$

56.1

1.8

13.9

13.4

4.5

5.4

2.8

2.0 
Tabie II

TRACE ELEMENT AVERAGES OF UIITS A-I

Averapes of Units

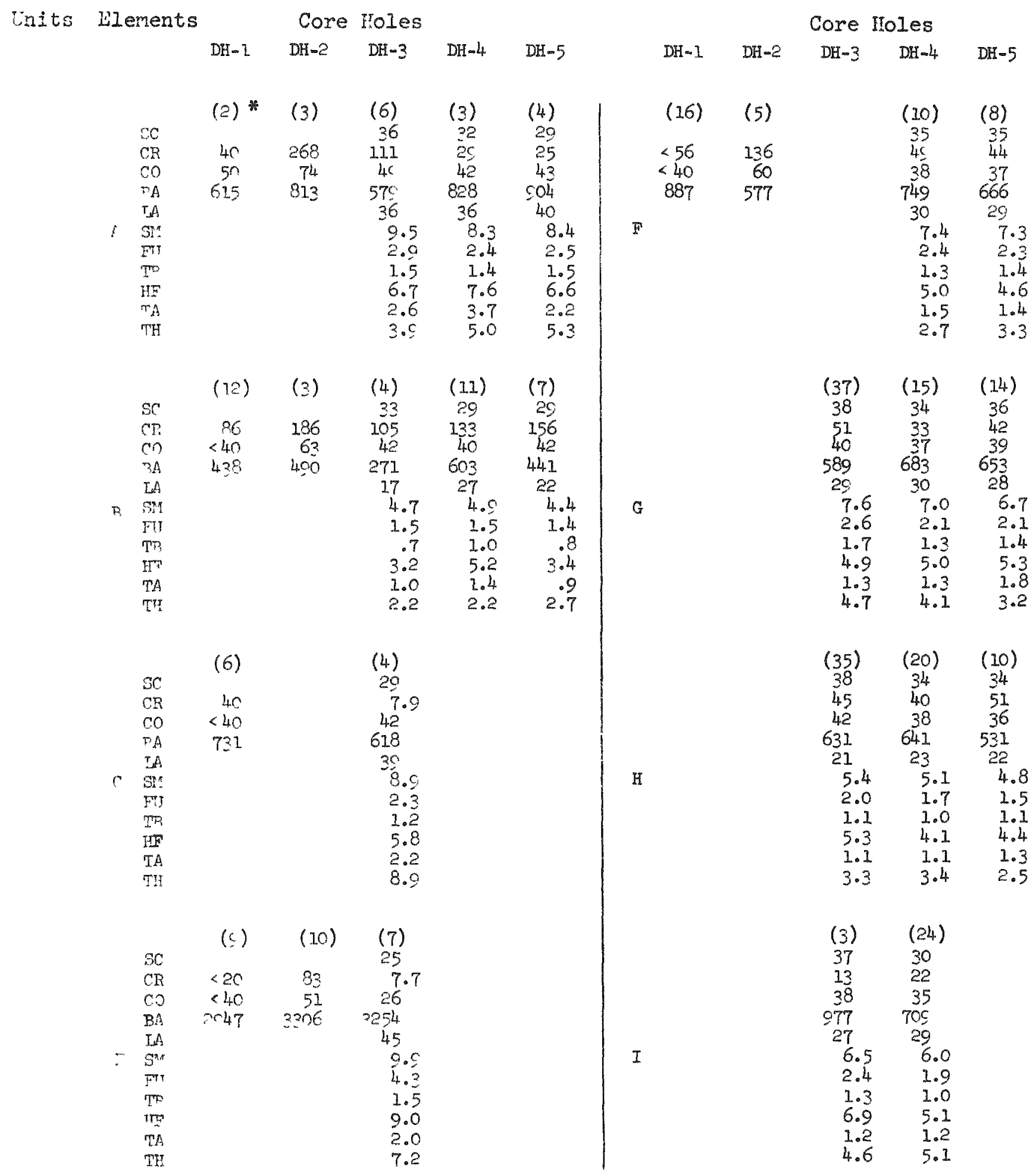

D CR 120 CO $<40$ BA 552

* Number in parenthesis is the number of averaged samples in the unit 


\section{TABLE III}

CORRELATIO COEFFICIENTS FOR ILAOR AND TRACT ELETENTS

SIO2 TIO2 AL2O3 FEO MNO MGO CAO NA2O KEO

$\begin{array}{llllllllllll}\text { SIO2 } & 1.00 & -.40 & .08 & -.41 & -.37 & -.54 & -.74 & .64 & .62\end{array}$

$\begin{array}{llllllllll}\text { TIO2 } & -.40 & 1.00 & . .76 & .89 & .23 & -.47 & -.13 & -.24 & .18\end{array}$

$\begin{array}{lllllllllll}\text { A1203 } & .08 & -.76 & 1.00 & -.84 & -.07 & .58 & .24 & .11 & -.21\end{array}$

$\begin{array}{lllllllllll}\text { FEO } & -.41 & .89 & . .84 & 1.00 & .18 & -.45 & -.17 & -.24 & .11\end{array}$

Maro $\quad \begin{array}{llllllllllllll}-.37 & .23 & -.07 & .18 & 1.00 & .01 & .29 & -.14 & 0.10\end{array}$

$\begin{array}{lllllllllllll}\text { MGO } & -.54 & -.47 & .58 & -.45 & .01 & 1.00 & .81 & -.56 & -.79\end{array}$

$\begin{array}{llllllllll}\mathrm{CAO} & -.74 & -.13 & .24 & -.17 & .29 & .81 & 1.00 & -.60 & -.84\end{array}$

$\begin{array}{llllllllll}\text { NA2O } & .64 & -.24 & .11 & -.24 & 0.14 & . .56 & -.60 & 1.00 & .54\end{array}$

$\begin{array}{llllllllll}120 & .62 & .18 & -.21 & .11 & -.10 & -.79 & -. .84 & .54 & 1.00\end{array}$

SC CR CO BA LA $\mathrm{BM}$ EU TB

$\begin{array}{llllllllllllllll}\text { SC } & 1.00 & -.03 & .35 & -.50 & -.53 & -.18 & -.21 & .21 & -.29 & -.20 & -.44\end{array}$

$\begin{array}{lllllllllllllll}C R & -.03 & 1.00 & .47 & -.42 & -.36 & -.48 & -.47 & -.48 & -.50 & -.25 & -.64\end{array}$

$\begin{array}{llllllllllllllll}C 0 & .35 & .47 & 1.00 & -.72 & -.14 & -.04 & -.38 & -.07 & -.25 & .25 & -.22\end{array}$

$\begin{array}{lllllllllllll}\text { BA } & -.50 & -.42 & -.72 & 1.00 & .57 & .54 & .84 & .38 & .74 & .22 & .51\end{array}$

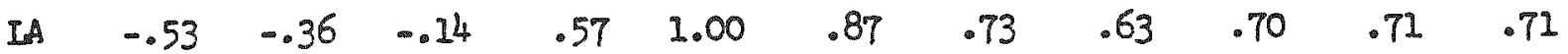

$\begin{array}{llllllllllllll}\text { SM } & -.18 & -.48 & -.04 & .54 & .87 & 1.00 & .87 & .80 & .78 & .72 & .72\end{array}$

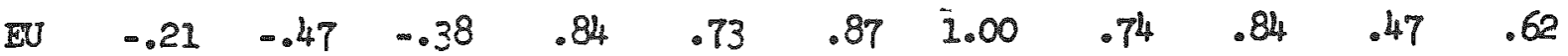

$\begin{array}{lllllllllllll} & .21 & -.48 & -.07 & .38 & .63 & .80 & .74 & 1.00 & .65 & .52 & .42\end{array}$

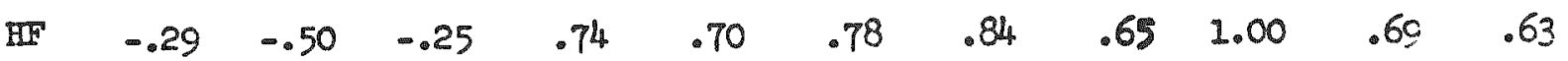

$\begin{array}{llllllllllllllll}\text { TA } & -. .20 & -. .25 & .25 & .22 & .71 & .72 & .47 & .52 & .69 & 1.00 & .46\end{array}$

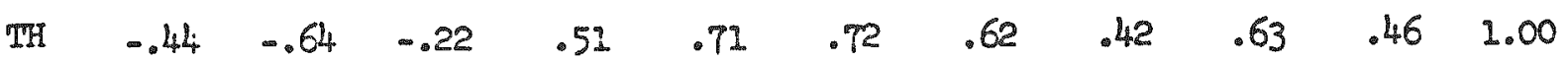


SOLIDIFICATION INDICES OF UNITS A-L

\begin{tabular}{|c|c|c|c|c|c|}
\hline Units & $\mathrm{DH}-1$ & $\mathrm{DH}-2$ & $\mathrm{DH}-3$ & $\mathrm{DH}-4$ & DH-5 \\
\hline A & 20.0 & 16.7 & 22.6 & 19.6 & 18.5 \\
\hline B & 33.0 & 32.9 & 34.6 & 33.5 & 35.5 \\
\hline$c$ & 18.4 & & 17.3 & & \\
\hline D & 33.8 & & & & \\
\hline$E$ & 13.3 & 11.7 & 15.0 & & \\
\hline $\mathrm{F}$ & 23.1 & 19.1 & & 21.8 & 20.9 \\
\hline G & & & 19.6 & 20.0 & 20.0 \\
\hline$H$ & & & 24.3 & 24.5 & 25.1 \\
\hline I & & & 17.1 & 16.9 & 19.0 \\
\hline $\mathrm{J}$ & & & 24.3 & 18.1 & 19.4 \\
\hline$K$ & & & 28.6 & 18.2 & 17.7 \\
\hline $\mathrm{L}$ & & & 19.8 & & \\
\hline
\end{tabular}




\section{TABLE $V$}

\section{STRATIGRAPHIC SECTION OF COLUMBIA RIVER BASALTS}

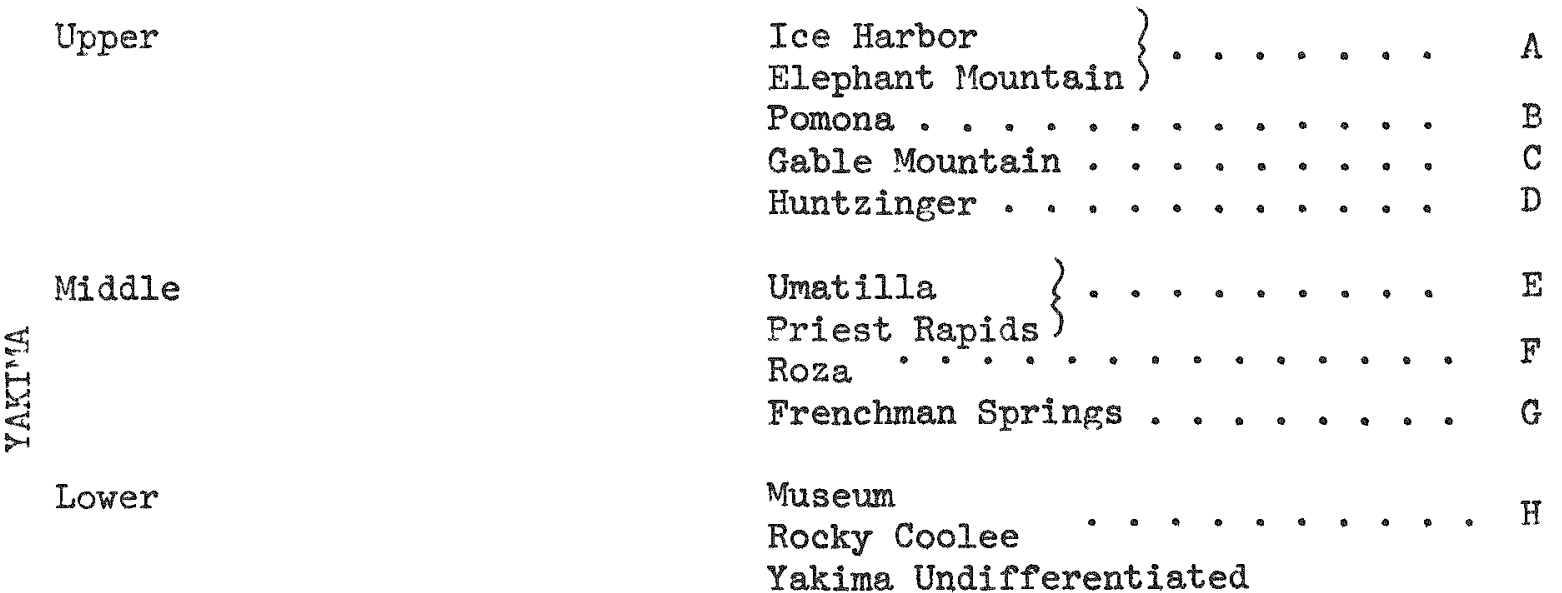

Picture Gorge and Lower Basalt 


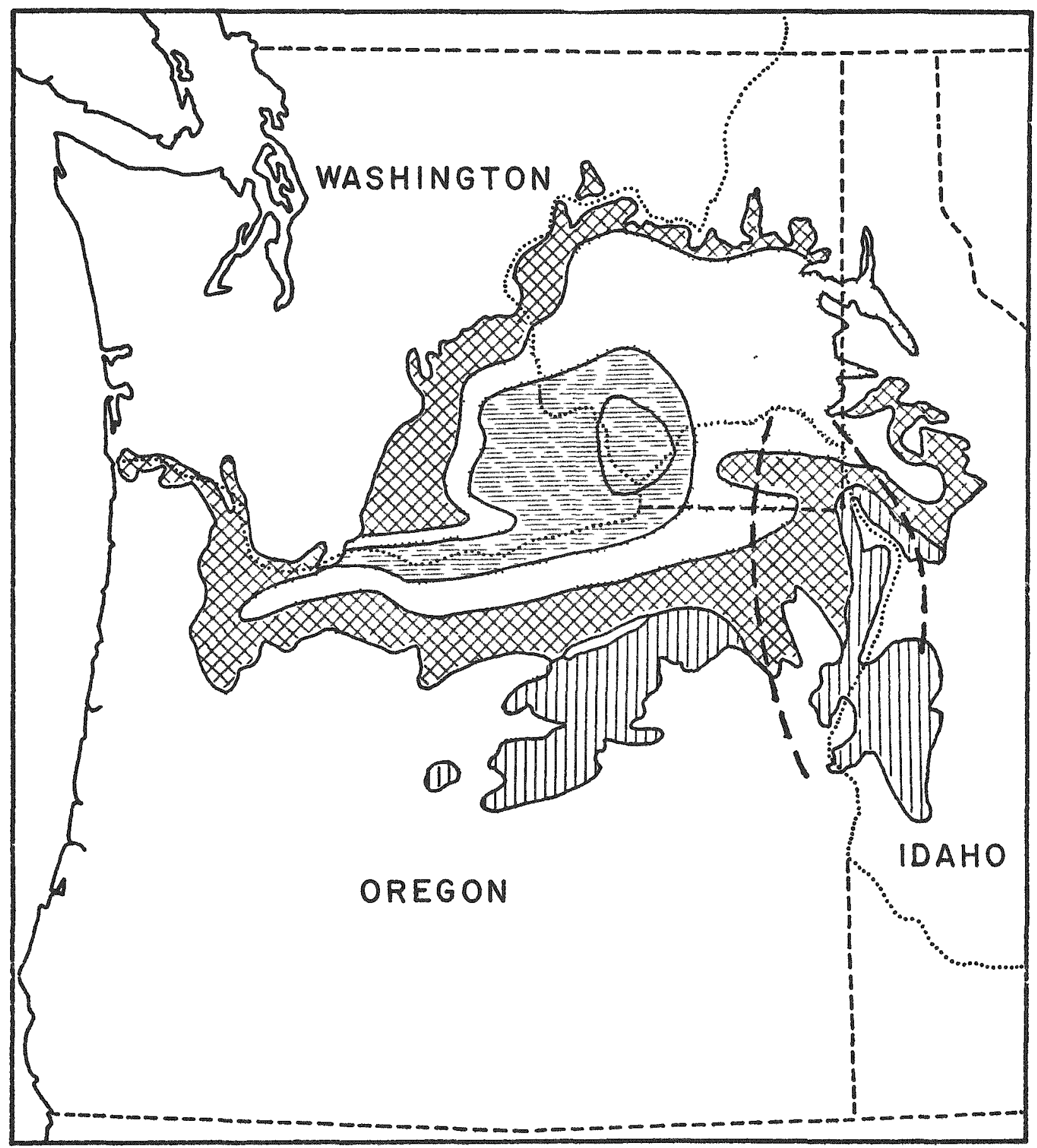

Upper Yakima kasalt

Midale Yakima basalt

Q8 Iower Yakima rasalt

IIIII Picture Gorge and Lower basalt

FIGURE 1

AREAL DISTRIBUTION OF COLUMBIA RIVER BASALT

[After Waters (1961), Wright, Groljer and Swanson (1973) and Taubeneck (1970).] 


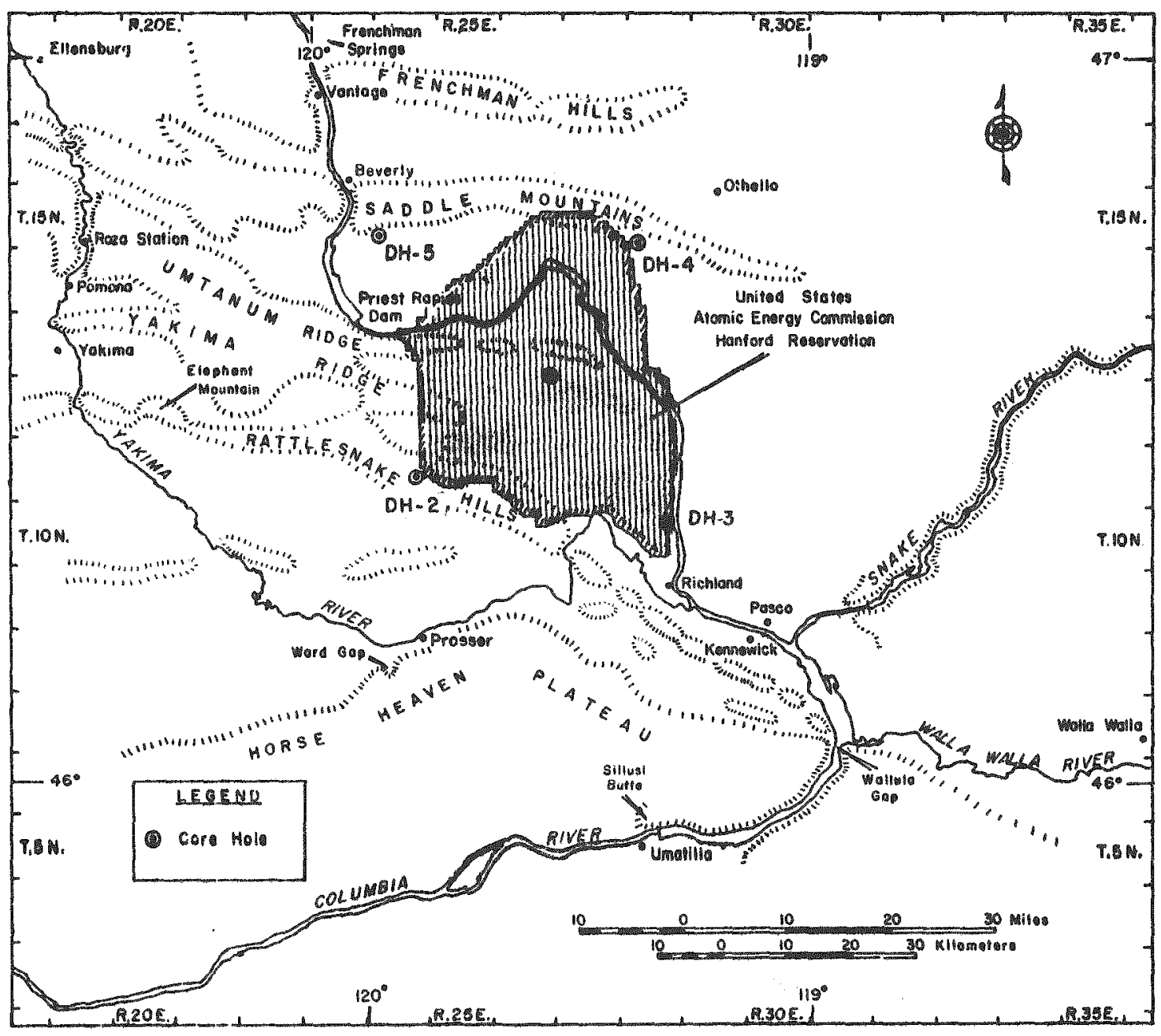

FIGURE 2

CORE HOLE LOCATION MAP

[After Brown (in press)] 

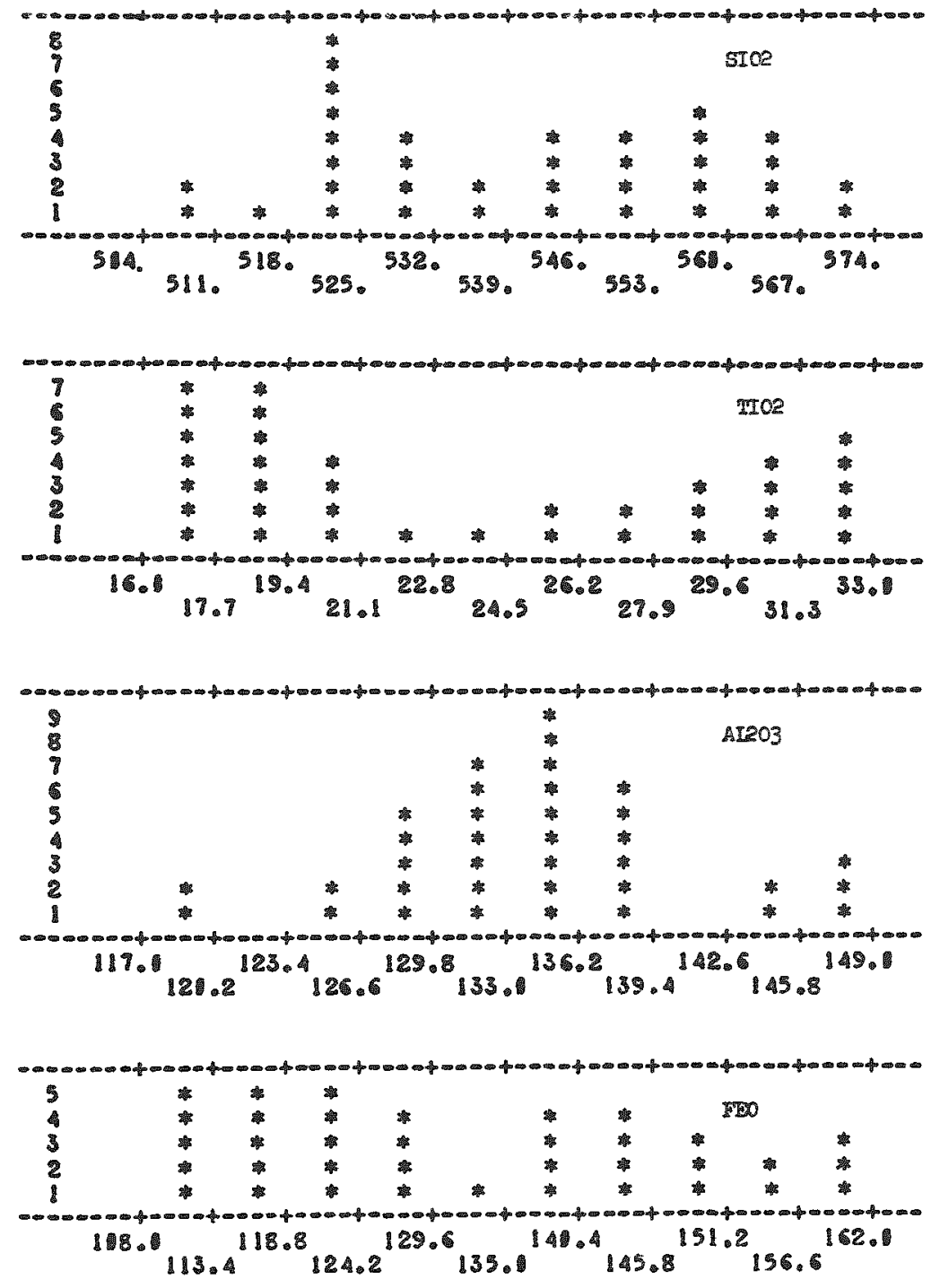

* rrote- decimal point is displaced one unit to the right of the true value.
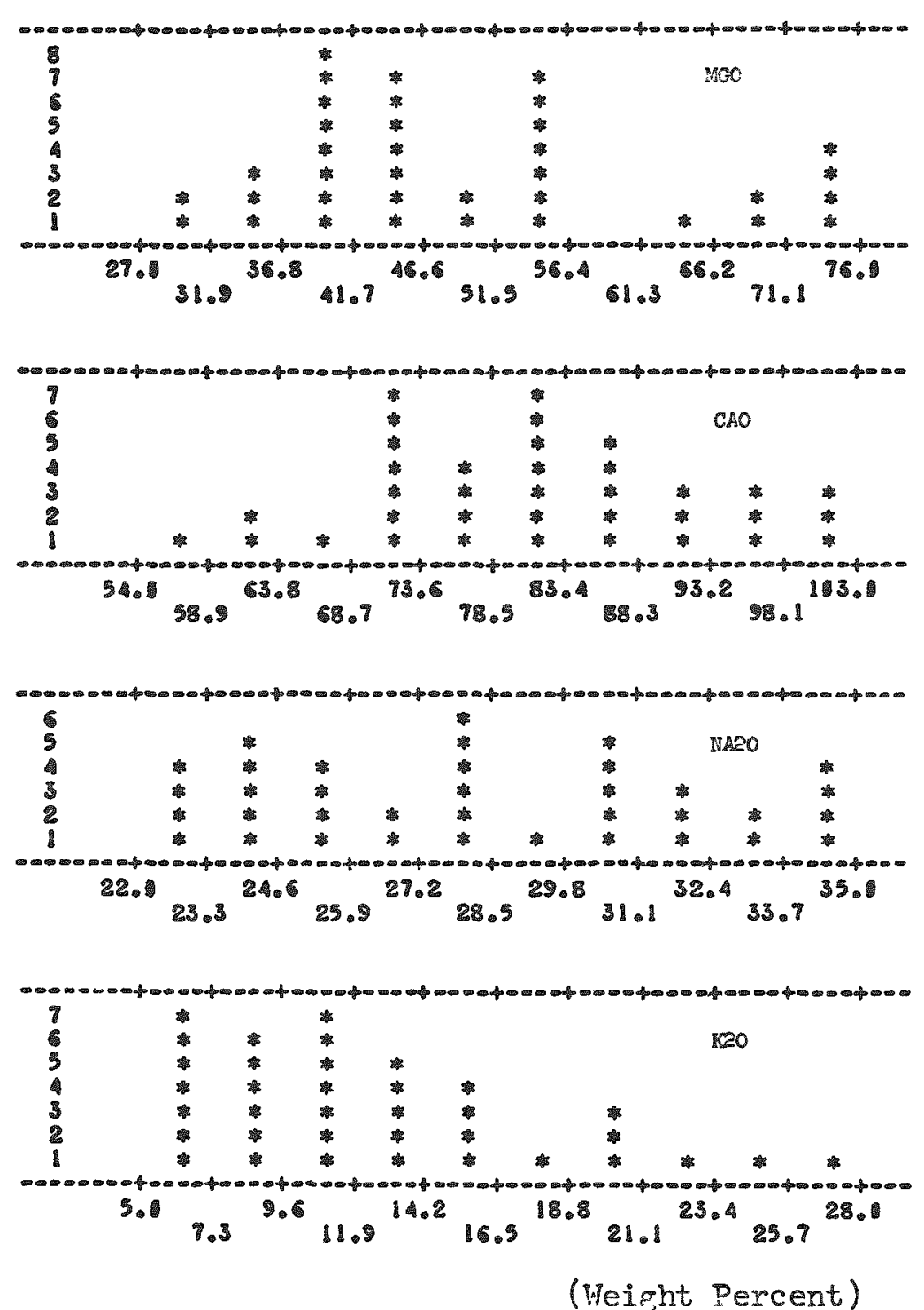

FIGURE 3 

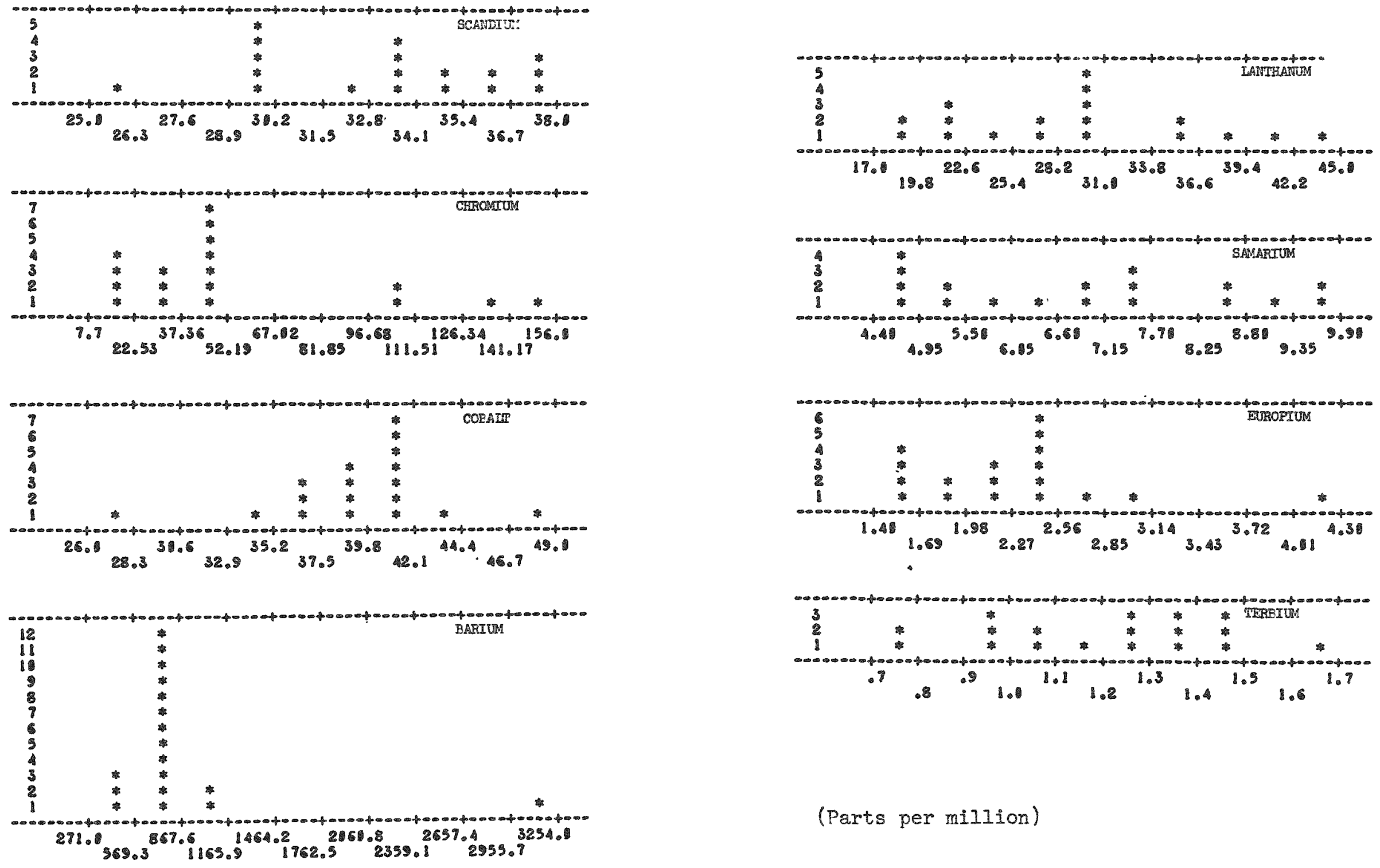

\section{(Parts per million)}

FIGURE 3 (Continued)

HISTOGRAMS OF AVERAGED GEOCHEMICAL DATA 

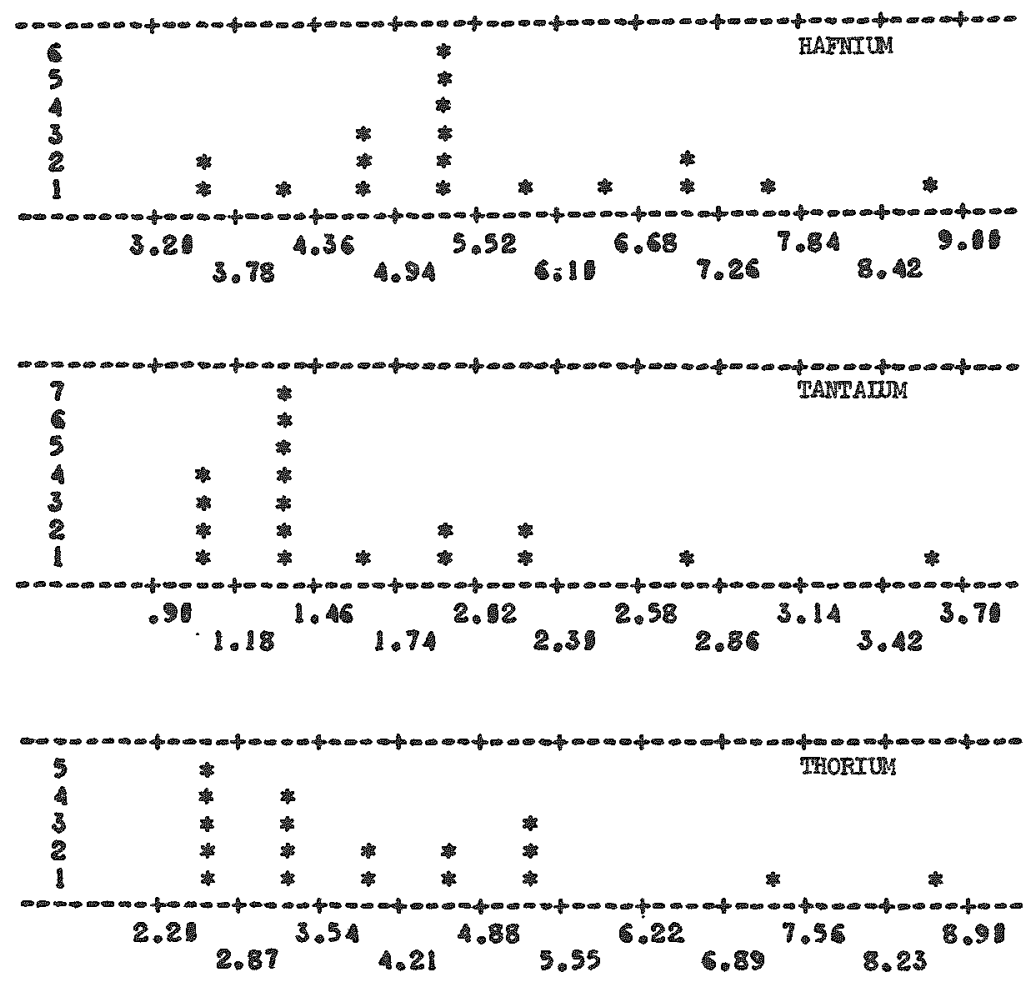

(Parts per million)

FIGURE 3 (Continued)

HISTOGRAMS OF AVERAGED GEOCHEMICAL DATA 


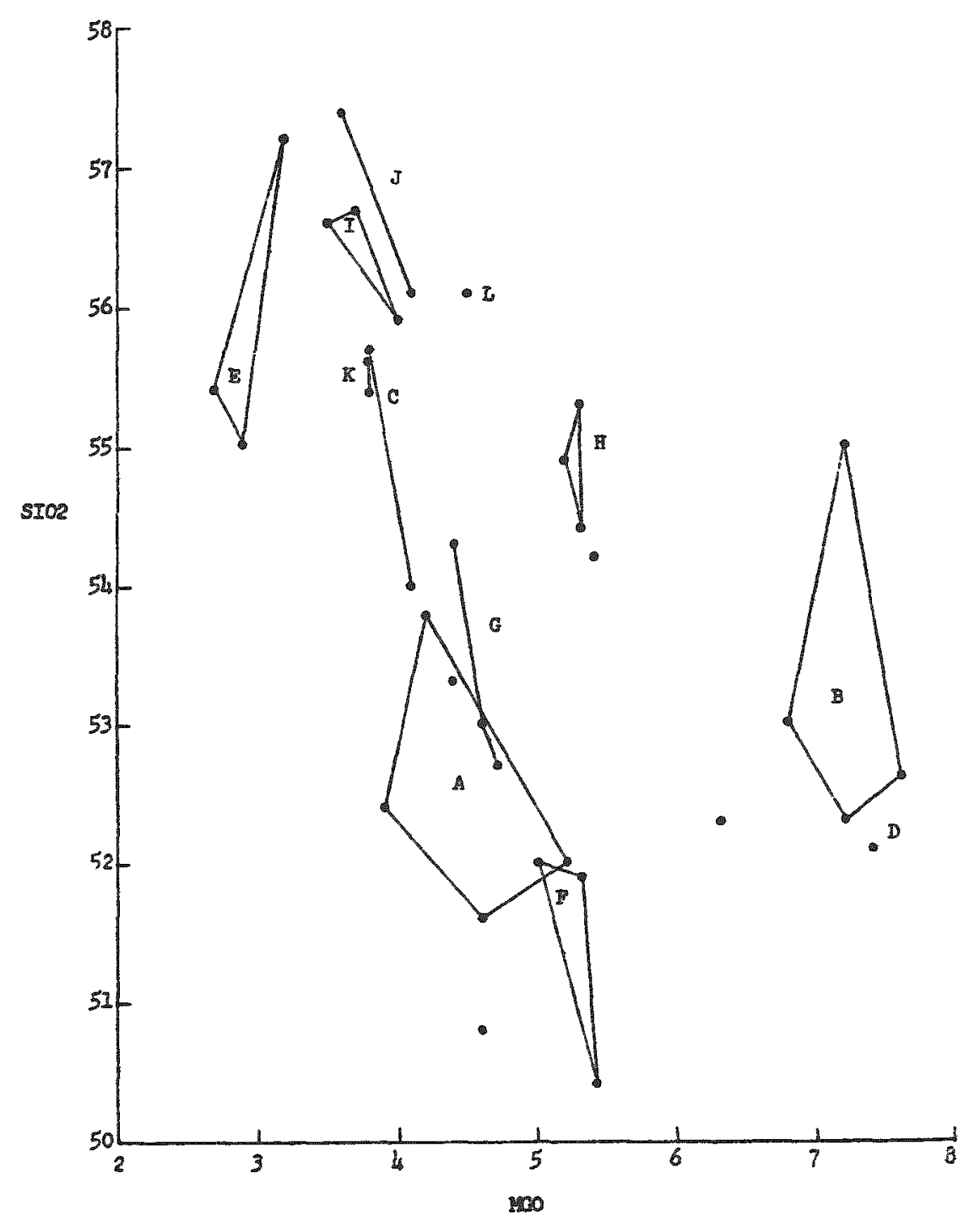

(Weight Percent)

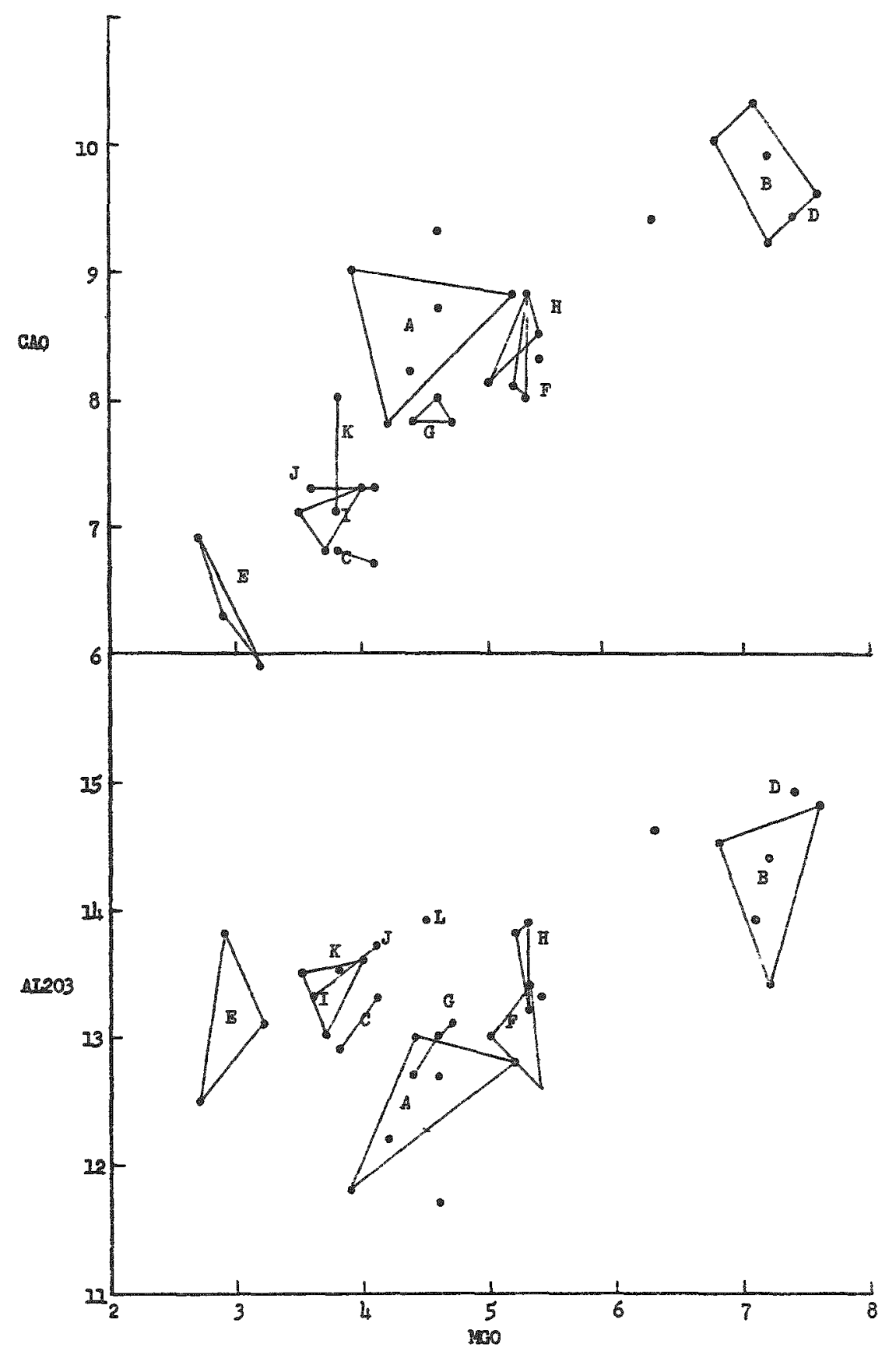

FIGURE 4 

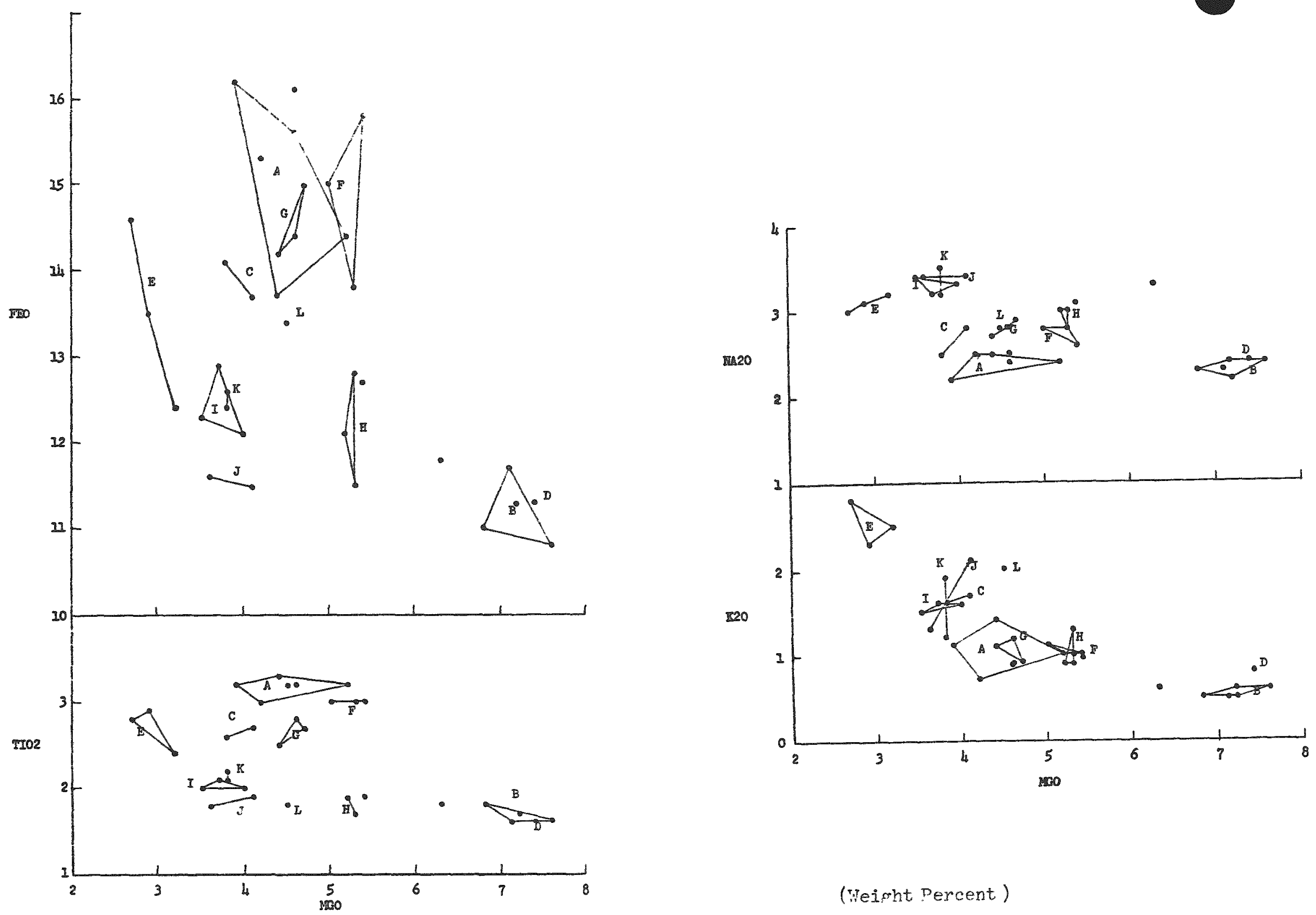

(Weight Percent)

FIGURE 4 (Continued)

Mg VARIATION DIAGRAM 


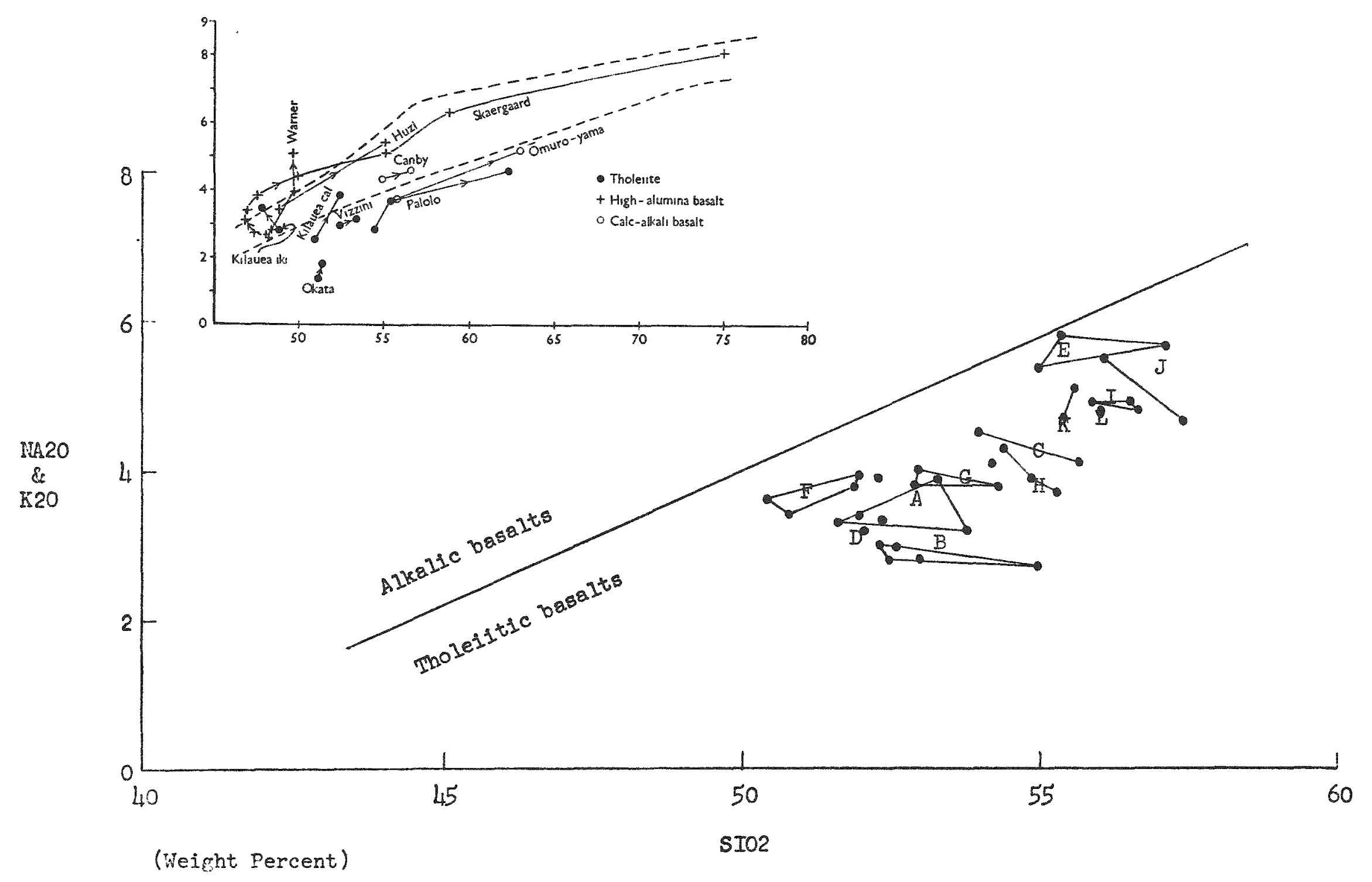

FIGURE 5

ALKALI-SILICA DIAGRAM 


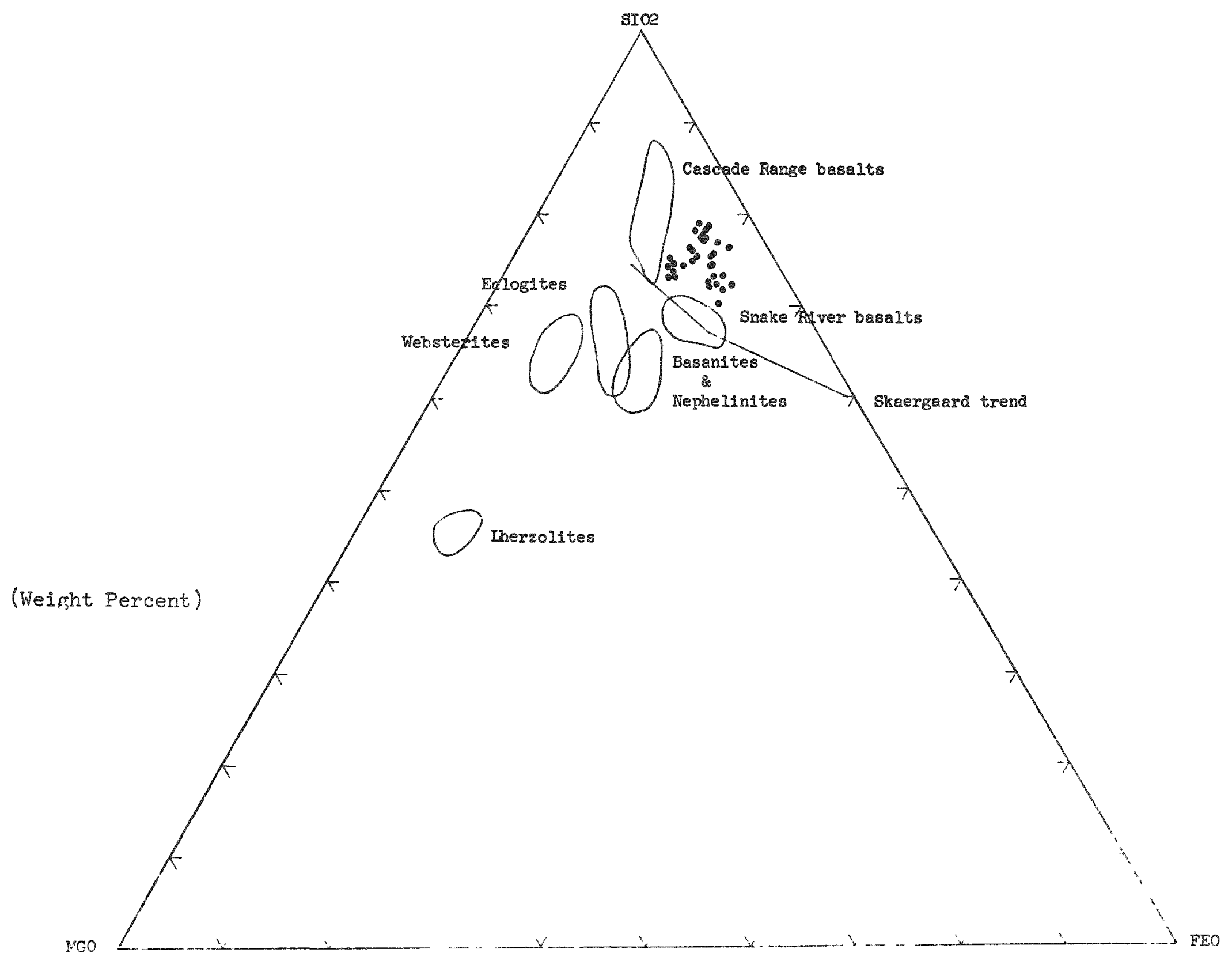

FIGURE 6

$\mathrm{SiO}_{2}-\mathrm{FeO}-\mathrm{MgO}$ DIAGRAM 


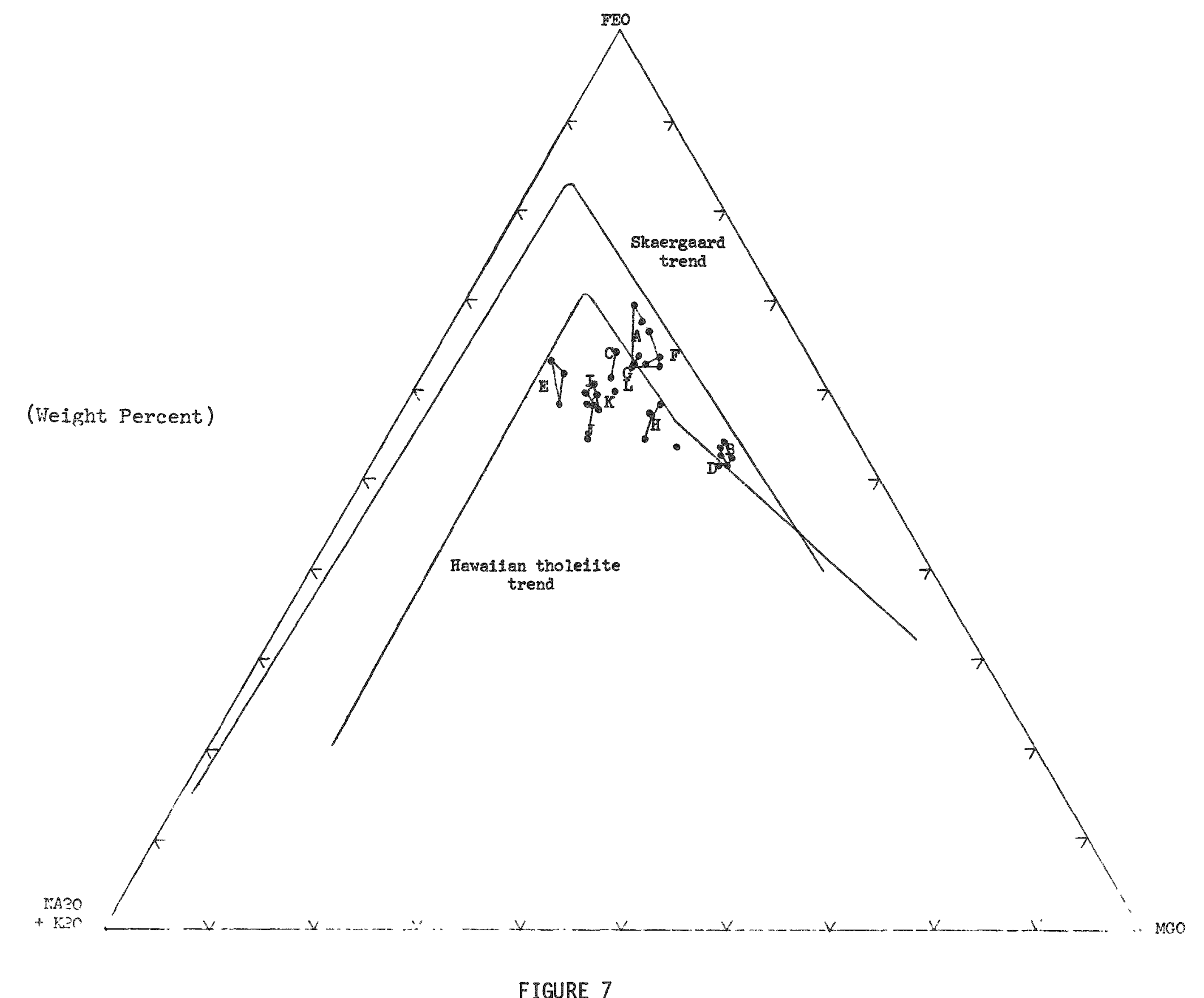

FIGURE 7

$\mathrm{Fe} 0-\mathrm{MgO}-\left(\mathrm{Na}_{2} \mathrm{O}\right.$ and $\left.\mathrm{K}_{2} \mathrm{O}\right)$ DIAGRAM 


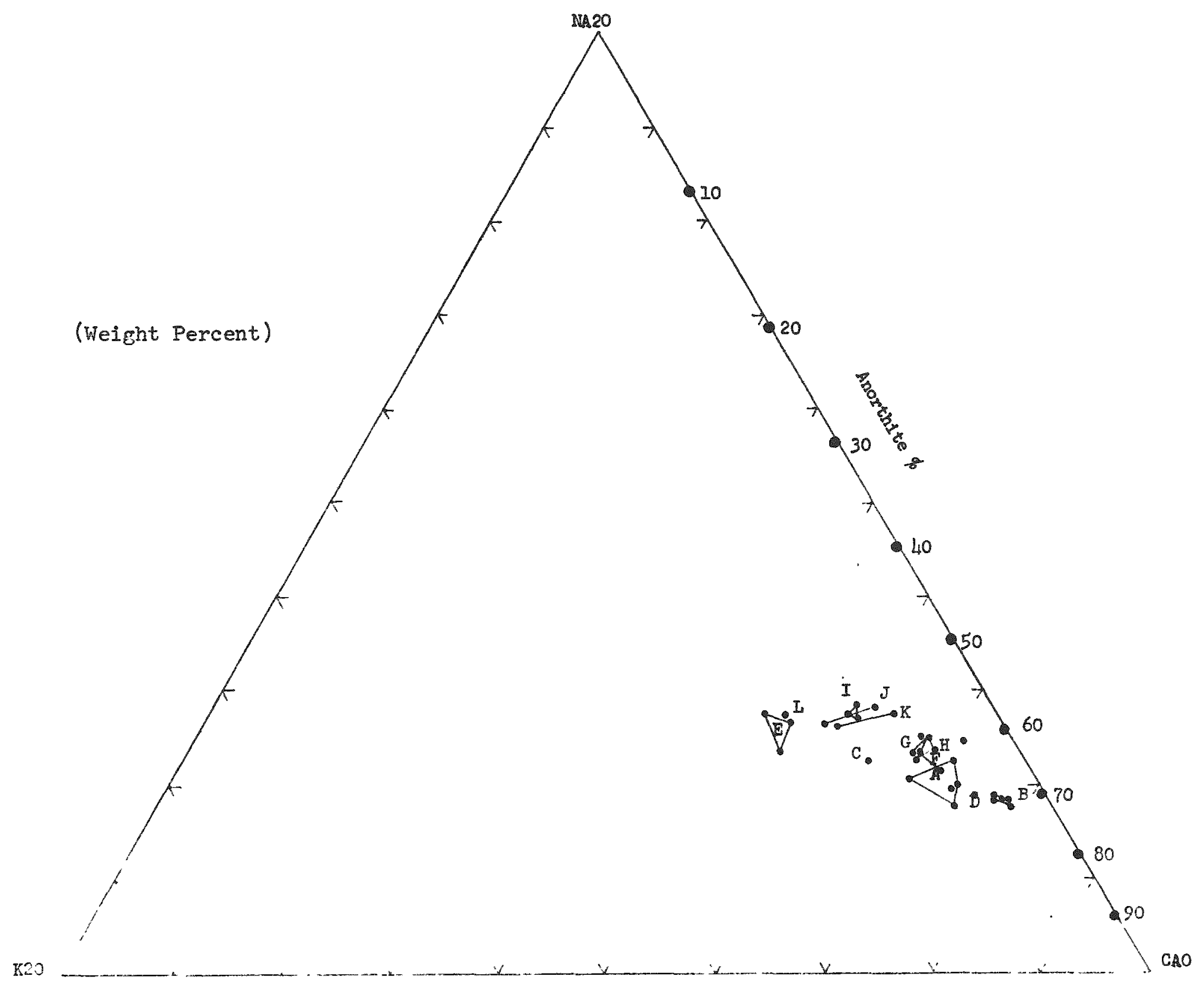

FIGURE 8

$\mathrm{Na}_{2} \mathrm{O}-\mathrm{CaO}-\mathrm{K}_{2} \mathrm{O}$ DIAGRAM 


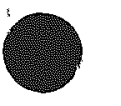

COLMBIF RIVFF EH JU

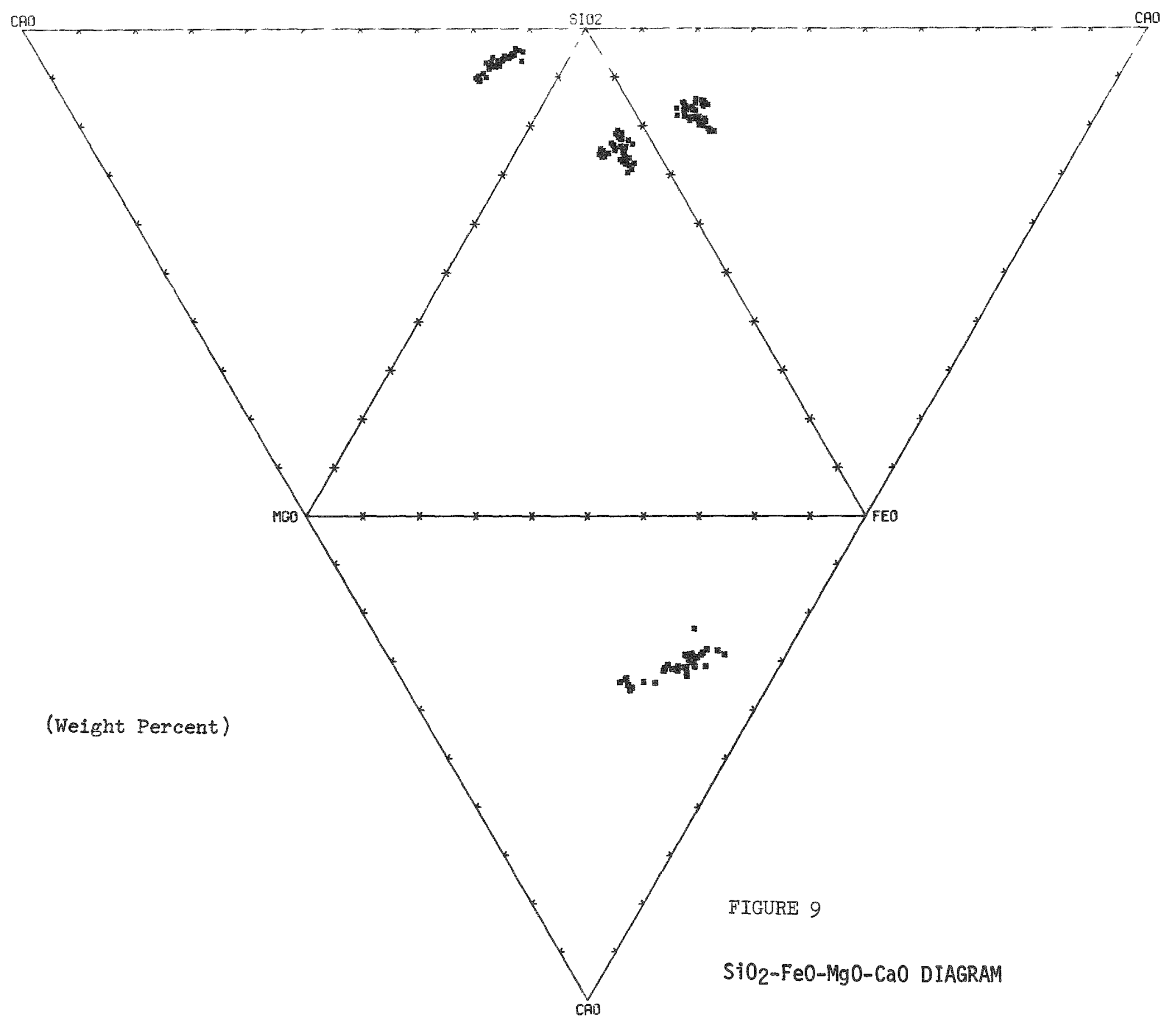


I JL11,1519 RIVEF BH.HLT.

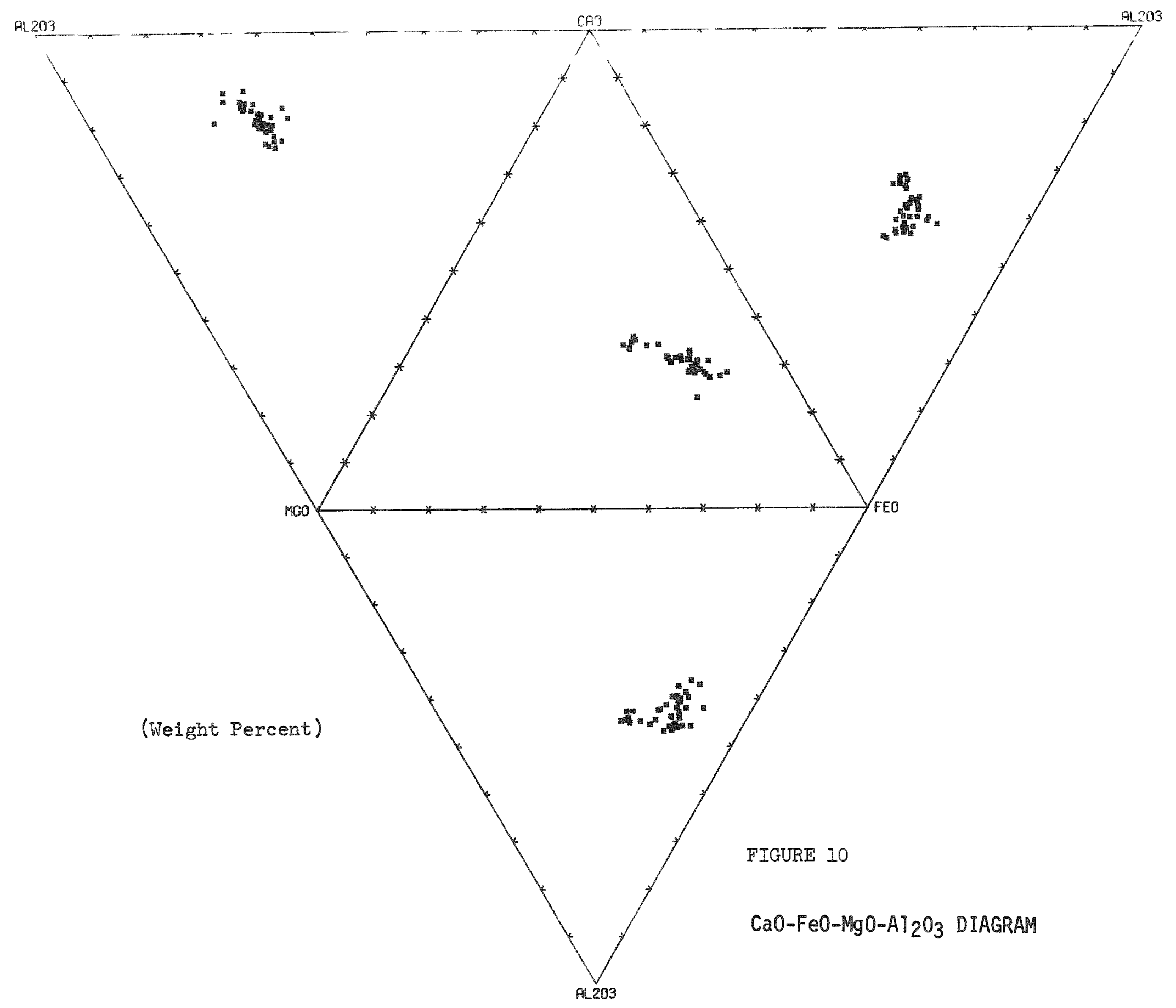




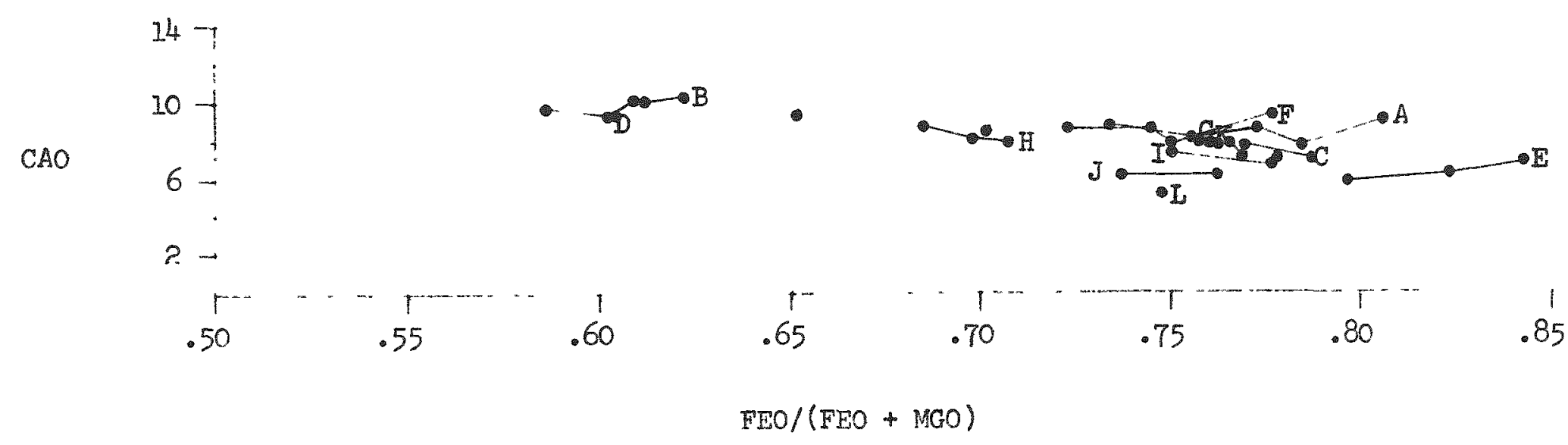

(Weight Percent)

\author{
FIGURE 11
}

IRON ENRICHMENT DIAGRAM 
(Weight Percent)

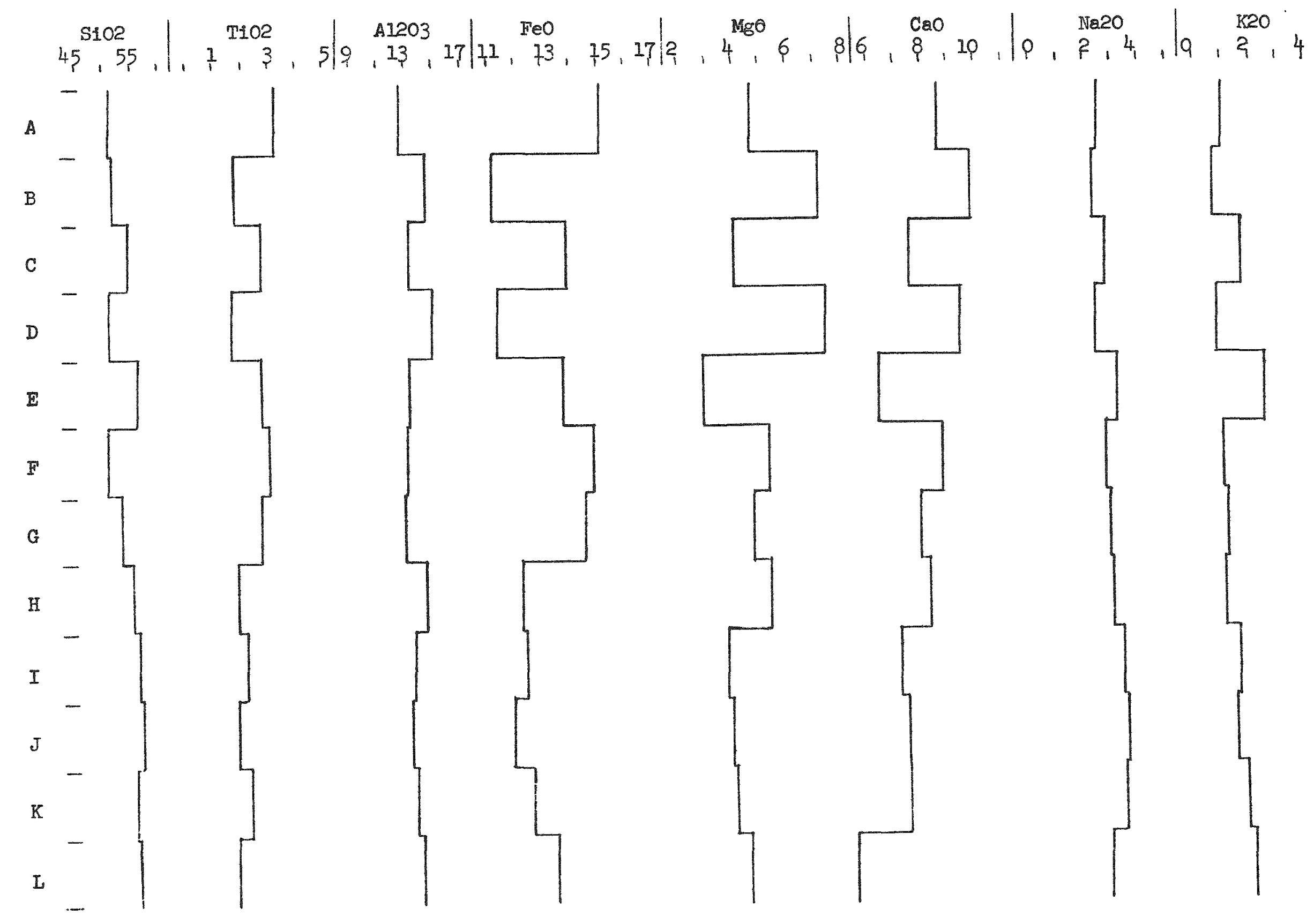

FIGURE 12

GEOCHEMICAL VARIATION OF COLUMBIA RIVER BASALT THROUGH TIME 